TITLE:

\title{
Asymptotic behaviour of certain families of harmonic bundles on Riemann surfaces
}

$\operatorname{AUTHOR}(S)$ :

Mochizuki, Takuro

CITATION:

Mochizuki, Takuro. Asymptotic behaviour of certain families of

harmonic bundles on Riemann surfaces. Journal of Topology 2016, 9(4): 1021-1073

ISSUE DATE:

2016-12

URL:

http://hdl.handle.net/2433/227916

\section{RIGHT:}

This is the peer reviewed version of the following article: [Mochizuki, T. (2016), Asymptotic behaviour of certain families of harmonic bundles on Riemann surfaces. Journal of Topology, 9: 1021-1073], which has been published in final form at https://doi.org/10.1112/jtopol/jtw018. This article may be used for non-commercial purposes in accordance with Wiley Terms and Conditions for Self-Archiving.; The full-text file will be made open to the public on 1 SEP 2017 in accordance with publisher's 'Terms and Conditions for Self-Archiving'; この論文は出版社版でありません。引用の際に は出版社版をご確認ご利用ください。; This is not the published version. Please cite only the published version. 


\title{
Asymptotic behaviour of certain families of harmonic bundles on Riemann surfaces
}

\author{
Takuro Mochizuki
}

\begin{abstract}
Let $\left(E, \bar{\partial}_{E}, \theta\right)$ be a stable Higgs bundle of degree 0 on a compact connected Riemann surface. Once we fix a flat metric $h_{\operatorname{det}(E)}$ on the determinant of $E$, we have the harmonic metrics $h_{t}(t>0)$ for the stable Higgs bundles $\left(E, \bar{\partial}_{E}, t \theta\right)$ such that $\operatorname{det}\left(h_{t}\right)=h_{\operatorname{det}(E)}$. We study the behaviour of $h_{t}$ when $t$ goes to $\infty$. First, we show that the Hitchin equation is asymptotically decoupled under the assumption that the Higgs field is generically regular semisimple. We apply it to the study of the so called Hitchin WKB-problem. Second, we study the convergence of the sequence $\left(E, \bar{\partial}_{E}, \theta, h_{t}\right)$ in the case rank $E=2$. We introduce a rule to determine the parabolic weights of a "limiting configuration", and we show the convergence of the sequence to the limiting configuration in an appropriate sense. The results can be appropriately generalized in the context of Higgs bundles with a Hermitian-Einstein metric on curves.
\end{abstract}

Keywords: harmonic bundle, asymptotic behaviour, asymptotic decoupling, Hitchin WKB-problem, limiting configuration, Hermitian-Einstein metric

MSC: $14 \mathrm{H} 60,53 \mathrm{C} 07$

\section{Introduction}

Let $X$ be a compact connected Riemann surface. Let $\left(E, \bar{\partial}_{E}, \theta\right)$ be a Higgs bundle of rank $r$ on $X$ with $\operatorname{deg}(E)=0$. Let $h$ be a Hermitian metric of $E$. We have the Chern connection $\nabla_{h}$ associated to $\left(E, \bar{\partial}_{E}, h\right)$. Let $R(h)$ denote the curvature of $\nabla_{h}$. Let $\theta_{h}^{\dagger}$ denote the adjoint of $\theta$ with respect to $h$. Recall the celebrated Hitchin equation [6]:

$$
R(h)+\left[\theta, \theta_{h}^{\dagger}\right]=0
$$

If the Hitchin equation is satisfied, $h$ is called a harmonic metric of $\left(E, \bar{\partial}_{E}, \theta\right)$, and $\left(E, \bar{\partial}_{E}, \theta, h\right)$ is called a harmonic bundle.

Remark 1.1 The Hitchin equation makes sense for a Higgs bundle with a Hermitian metric on any complex curve. In this introduction, $X$ is assumed to be compact to simplify the explanation.

If $\operatorname{deg}(E)$ is not 0 , we take a Hermitian metric $h_{\operatorname{det}(E)}$ of the determinant line bundle $\operatorname{det}(E)$, and we consider the Hermitian-Einstein condition $R(h)^{\perp}+\left[\theta, \theta_{h}^{\dagger}\right]=0$, where $R(h)^{\perp}$ is the trace-free part of $R(h)$. The condition is also called the Hitchin equation. In this introduction, we assume $\operatorname{deg}(E)=0$ for simplicity.

If $\operatorname{rank} E=1$, we always have $\left[\theta, \theta_{h}^{\dagger}\right]=0$. Hence, the Hitchin equation is reduced to $R(h)=0$, i.e., the metric $h$ is flat with respect to the Chern connection. By the classical harmonic theory, we can always find such a harmonic metric in the rank one case, which is unique up to the multiplication of positive constants.

In the higher rank case, we fix a harmonic metric $h_{\operatorname{det} E}$ of $\left(\operatorname{det}(E), \bar{\partial}_{\operatorname{det} E}, \operatorname{tr} \theta\right)$. According to Hitchin [6] and Simpson [19], if the Higgs bundle $\left(E, \bar{\partial}_{E}, \theta\right)$ is stable, we have a unique harmonic metric $h$ of $\left(E, \bar{\partial}_{E}, \theta\right)$ such that $\operatorname{det}(h)=h_{\operatorname{det} E}$.

For any non-zero complex number $t$, the Higgs bundle $\left(E, \bar{\partial}_{E}, t \theta\right)$ is also stable. We obtain a family of harmonic metrics $h_{t}\left(t \in \mathbb{C}^{*}\right)$ satisfying $\operatorname{det}\left(h_{t}\right)=h_{\operatorname{det} E}$. It is easy to observe that $h_{t_{1}}=h_{t_{2}}$ if $\left|t_{1}\right|=\left|t_{2}\right|$. So, it is enough to consider the case where $t$ are positive numbers.

Simpson studied the behaviour of $\left(E, \bar{\partial}_{E}, t \theta, h_{t}\right)$ when $t \rightarrow 0$. (See $[19,20,21,22]$, for example.) He discovered the convergence to a polarized variation of Hodge structure, and he gave various applications of this interesting phenomena. 
More recently, there has been a growing interest to the behaviour of $\left(E, \bar{\partial}_{E}, t \theta, h_{t}\right)$ when $t \rightarrow \infty$. In [7], Katzarkov, Noll, Pandit and Simpson proposed "Hitchin WKB-problem" on the behaviour of the family of harmonic metrics $h_{t}$ and the monodromy of the associated flat connections $\nabla_{h}+\theta+\theta_{h}^{\dagger}$, in relation with their magnificent theory of harmonic maps to buildings. In [11, 12], Mazzeo, Swoboda, Weiss and Witt studied the rank 2 case under the assumption that the zeroes of $\operatorname{det}(\theta)-(\operatorname{tr} \theta)^{2} / 4$ are simple, i.e., the spectral curve of the Higgs field is smooth irreducible and simply ramified over $X$, motivated by the study on the structure of the end of the moduli spaces of Higgs bundles. They introduced the concept of "limiting configuration", and they proved the convergence to the limiting configuration under the assumption, inspired by the work of Gaiotto, Moore and Neitzke [4, 5]. In [3], Collier and Li closely studied the issue for some Toda-like harmonic bundles in a rather explicit way, and they resolved Hitchin WKB-problem in these cases for some kind of non-critical paths.

In this paper, we shall give two results on the asymptotic behaviour of the harmonic bundles $\left(E, \bar{\partial}_{E}, t \theta, h_{t}\right)$ $(t \rightarrow \infty)$. One is the asymptotic decoupling, and the other is the convergence to the limiting configuration for harmonic bundles of rank two.

\subsection{Asymptotic decoupling}

The Hitchin equation is much simplified if $R(h)=\left[\theta, \theta_{h}^{\dagger}\right]=0$ holds. The equation $R(h)=0$ implies that $\left(E, \nabla_{h}, h\right)$ is a unitary flat bundle. The additional condition $\left[\theta, \theta_{h}^{\dagger}\right]=0$ implies that, at least locally, we have a flat decomposition $(E, \nabla, h)=\bigoplus_{i=1}^{r}\left(E_{i}, \nabla_{i}, h_{i}\right)$ into flat line bundles such that $\theta=\bigoplus \phi_{i} \cdot \mathrm{id}_{E_{i}}$, where $\phi_{i}$ are holomorphic one forms. In [11], this kind of simplification seems to be called "decoupling" of the Hitchin equation.

Our first purpose is to show that if $t$ is sufficiently large, the Hitchin equation for $\left(E, \bar{\partial}_{E}, t \theta\right)$ is almost decoupled in some sense.

\subsubsection{Generically regular semisimple Higgs bundles}

To state the claim more precisely, we introduce a condition for Higgs bundles. Let $\left(E, \bar{\partial}_{E}, \theta\right)$ be a Higgs bundle on $X$. We have the associated coherent sheaf $M_{E, \theta}$ on the cotangent bundle $T^{*} X$. The support $\Sigma(E, \theta)$ is called the spectral curve of the Higgs bundle. The number of the points of $\rho(P):=T_{P}^{*} X \cap \Sigma(E, \theta)$ are finite for any $P \in X$. We say that the Higgs bundle $\left(E, \bar{\partial}_{E}, \theta\right)$ is generically regular semisimple if the following holds:

- We have a discrete subset $D \subset X$ such that $\rho(P)=\operatorname{rank} E$ for any $P \in X \backslash D$.

Let $D(E, \theta)$ denote the set of the points $P \in X$ such that $\rho(P)<\operatorname{rank} E$, which we call the discriminant of the Higgs bundle.

Suppose that $\left(E, \bar{\partial}_{E}, \theta, h\right)$ is generically regular semisimple. Then, the following holds for any point $P \in$ $X \backslash D(E, \theta)$ with a small neighbourhood $U_{P}$ of $P$ :

- We have holomorphic 1-forms $\phi_{P, 1}, \ldots, \phi_{P, r}$ on $U_{P}$ and a decomposition of the Higgs bundle

$$
\left(E, \bar{\partial}_{E}, \theta\right)_{\mid U_{P}}=\bigoplus_{i=1}^{r}\left(E_{P, i}, \bar{\partial}_{E_{P, i}}, \phi_{P, i} \operatorname{id}_{E_{P, i}}\right)
$$

where we assume that $\operatorname{rank} E_{i}=1(i=1, \ldots, r)$, and that $\phi_{P, i}-\phi_{P, j}(i \neq j)$ have no zero.

\subsubsection{Asymptotic decoupling}

Let $\left(E, \bar{\partial}_{E}, \theta\right)$ be a stable Higgs bundle of degree 0 on $X$. Suppose that it is generically regular semisimple. We take any Kähler metric $g_{X}$ of $X$. For any local section $s$ of $\operatorname{End}(E) \otimes \Omega^{p, q}$, we have the function $|s|_{h_{t}, g_{X}}: X \longrightarrow \mathbb{R}$, where $|s|_{h_{t}, g_{X}}(P)(P \in X)$ are the norm of $s_{\mid P}$ with respect to $h_{t}$ and $g_{X}$. We have the asymptotic decoupling in the following sense. 
Theorem 1.2 (Theorem 2.9) We take any neighbourhood $N$ of the discriminant $D(E, \theta)$. Then, there exist positive constants $C_{0}$ and $\epsilon_{0}$ such that the following holds on $X \backslash N$ :

$$
\left|R\left(h_{t}\right)\right|_{h_{t}, g_{X}}=|t|^{2}\left|\left[\theta, \theta_{h_{t}}^{\dagger}\right]\right|_{h_{t}, g_{X}} \leq C_{0} \exp \left(-\epsilon_{0} t\right)
$$

The constants $C_{0}$ and $\epsilon_{0}$ may depend only on $\left(X, g_{X}\right), N$ and $\Sigma(E, \theta)$.

We also have the family of flat connections $\mathbb{D}_{h_{t}}^{1}:=\nabla_{h_{t}}+t \theta+(t \theta)_{h_{t}}^{\dagger}$, which are correctly associated to the harmonic bundles $\left(E, \bar{\partial}_{E}, t \theta, h_{t}\right)$. Let us describe that we have nice approximations of these connections.

Let $P$ be any point of $X \backslash D(E, \theta)$. We take a small neighbourhood $U_{P}$ of $P$ in $X \backslash D(E, \theta)$. We have a decomposition of the Higgs bundle $\left(E, \bar{\partial}_{E}, \theta\right)_{\mid U_{P}}=\bigoplus_{i=1}^{r}\left(E_{P, i}, \bar{\partial}_{E_{P, i}}, \theta_{P, i}\right)$, where rank $E_{P, i}=1$. Let $h_{t, E_{P, i}}$ be the restriction of $h_{t}$ to $E_{P, i}$. By taking the direct sum, we obtain a Hermitian metric $h_{t, P, 0}:=\bigoplus_{i=1}^{r} h_{t, E_{P, i}}$ of $E_{\mid U_{P}}$. Note that Theorem 1.2 implies the following.

Lemma 1.3 We have positive constants $C_{P, 0}^{\prime}$ and $\epsilon_{P, 0}^{\prime}$ such that the following estimate holds for any local sections $u_{i}$ and $u_{j}(i \neq j)$ of $E_{P, i}$ and $E_{P, j}$ :

$$
\left|h_{t}\left(u_{i}, u_{j}\right)\right| \leq C_{P, 0}^{\prime} \exp \left(-\epsilon_{P, 0}^{\prime} t\right)\left|u_{i}\right|_{h_{t}}\left|u_{j}\right|_{h_{t}}
$$

In particular, we have a constant $K_{P}>1$ such that $K_{P}^{-1} h_{t, P, 0} \leq h_{t \mid U_{P}} \leq K_{P} h_{t, P, 0}$ for any $t>1$.

By varying $P \in X \backslash D(E, \theta)$ and by gluing $h_{t, P, 0}$, we obtain a family of Hermitian metrics $h_{t, 0}(t>1)$ on $E_{\mid X \backslash D(E, \theta)}$. We have the Chern connection $\nabla_{t, 0}$ of $\left(E_{\mid X \backslash D(E, \theta)}, h_{t, 0}\right)$. Let $(t \theta)_{h_{t, 0}}^{\dagger}$ denote the adjoint of $t \theta_{\mid X \backslash D(E, \theta)}$ with respect to $h_{t, 0}$. We set $\mathbb{D}_{h_{t, 0}}^{1}:=\nabla_{t, 0}+t \theta+(t \theta)_{h_{t, 0}}^{\dagger}$.

Theorem 1.4 (Proposition 2.12, Corollary 2.13) Take any neighbourhood $N$ of $D(E, \theta)$. Then, we have a constant $K>1$ such that $K^{-1} h_{t, 0 \mid X \backslash N} \leq h_{t \mid X \backslash N} \leq K h_{t, 0 \mid X \backslash N}$ for any $t>1$. We also have positive constants $C_{1}$ and $\epsilon_{1}$ such that the following holds on $X \backslash N$ :

$$
\left|R\left(h_{t, 0}\right)\right|_{h_{t}, g_{X}} \leq C_{1} \exp \left(-\epsilon_{1} t\right), \quad\left|\mathbb{D}_{h_{t}}^{1}-\mathbb{D}_{h_{t, 0}}^{1}\right|_{h_{t}, g_{X}} \leq C_{1} \exp \left(-\epsilon_{1} t\right)
$$

The constants $C_{1}$ and $\epsilon_{1}$ may depend only on $\left(X, g_{X}\right), N$ and $\Sigma(E, \theta)$.

We note that we can obtain these estimates in an elementary way which is standard in the study of the asymptotic behaviour of harmonic bundles around the singularity, pioneered by Simpson [20], and pursued further by the author $[13,16]$. We also emphasize that we can obtain these kinds of estimates without the assumption that harmonic bundles are given on a compact Riemann surface. Indeed, we shall study harmonic bundles given on discs in $\S 2$, which is clearly enough for the above estimates on relatively compact regions.

Finally, we remark that the estimates can be generalized in the case $\operatorname{deg}(E) \neq 0$, i.e., in the context of Higgs bundles with Hermitian-Einstein metrics on curves. (See $\S 2.5$.)

\subsubsection{Hitchin WKB-problem}

Together with a rather standard argument of singular perturbations, we can apply Theorem 1.4 to the Hitchin WKB-problem in [7].

We recall a notation in [7]. Let $V$ be an $r$-dimensional complex vector space. For Hermitian metrics $h_{1}, h_{2}$, we can take a base $e_{1}, \ldots, e_{r}$ of $V$ which is orthogonal with respect to both $h_{i}(i=1,2)$. We have the real numbers $\kappa_{j}(j=1, \ldots, r)$ determined by $\kappa_{j}:=\log \left|e_{j}\right|_{h_{2}}-\log \left|e_{j}\right|_{h_{1}}$. We impose $\kappa_{1} \geq \kappa_{2} \geq \cdots \geq \kappa_{r}$. Then, we set

$$
\vec{d}\left(h_{1}, h_{2}\right):=\left(\kappa_{1}, \ldots, \kappa_{r}\right) \in \mathbb{R}^{r} .
$$

Let us return to the study on the family of harmonic bundles $\left(E, \bar{\partial}_{E}, t \theta, h_{t}\right)(t>0)$ for a stable Higgs bundle $\left(E, \bar{\partial}_{E}, \theta\right)$ of rank $r$ with $\operatorname{deg}(E)=0$ on a compact Riemann surface $X$, which is generically regular semisimple. We take a universal covering $\pi: Y \longrightarrow X \backslash D(E, \theta)$. Then, we have the decomposition of the Higgs bundle $\pi^{*}\left(E, \bar{\partial}_{E}, \theta\right)=\bigoplus_{i=1}^{r}\left(E_{i}, \bar{\partial}_{E_{i}}, \phi_{i} \operatorname{id}_{E_{i}}\right)$, where $\phi_{i}$ are holomorphic 1-forms. We have $\operatorname{rank} E_{i}=1$, and $\phi_{i}-\phi_{j}$ $(i \neq j)$ have no zeroes. 
Let $[0,1]$ denote the closed interval $\{0 \leq s \leq 1\}$. Let $\gamma:[0,1] \longrightarrow Y$ be a $C^{\infty}$-path. We have the expressions $\gamma^{*}\left(\phi_{i}\right)=a_{i} d s$ where $a_{i}$ are $C^{\infty}$-functions on $[0,1]$. The path $\gamma$ is called non-critical if $\operatorname{Re} a_{i}(s) \neq \operatorname{Re} a_{j}(s)$ $(i \neq j)$ for any $s$. In that case, we may assume $\operatorname{Re} a_{i}(s)<\operatorname{Re} a_{j}(s)(i<j)$. We set

$$
\alpha_{i}:=-\int_{0}^{1} \operatorname{Re}\left(a_{i}\right) d s .
$$

We have the families of Hermitian metrics $h_{t, \gamma(\kappa)}(t>0)$ on the fibers $E_{\gamma(\kappa)}(\kappa=0,1)$, induced by the harmonic metrics $h_{t}$. Let $\Pi_{\gamma, t}: E_{\gamma(0)} \longrightarrow E_{\gamma(1)}$ denote the parallel transport of the flat connection $\mathbb{D}_{h_{t}}^{1}$ along $\gamma$. Let $\Pi_{\gamma, t}^{*} h_{t, \gamma(1)}$ denote the family of Hermitian metrics on $E_{\gamma(0)}$ induced by $h_{t, \gamma(1)}$ and $\Pi_{\gamma, t}$.

Theorem 1.5 (Theorem 2.17) If $\gamma$ is non-critical, there exist positive constants $C_{2}$ and $\epsilon_{2}$ such that the following holds:

$$
\left|\frac{1}{t} \vec{d}\left(h_{t, \gamma(0)}, \Pi_{\gamma, t}^{*} h_{t, \gamma(1)}\right)-\left(2 \alpha_{1}, \ldots, 2 \alpha_{r}\right)\right| \leq C_{2} \exp \left(-\epsilon_{2} t\right)
$$

The constants $C_{2}$ and $\epsilon_{2}$ may depend only on $X, \phi_{1}, \ldots, \phi_{r}$ and $\gamma$.

The theorem was conjectured in [7], and a different version of the problem called the Riemann-Hilbert WKB-problem was studied in detail. Some cases were verified in [3]. (See [3, 7] for the precise statements.) We emphasize that we can obtain this kind of estimate for more general families of harmonic bundles given on complex curves which are not necessarily compact.

\subsection{Convergence to the limiting configuration}

\subsubsection{Limiting configuration}

Let $\left(E, \bar{\partial}_{E}, \theta\right)$ be a stable Higgs bundle of rank $r$ with $\operatorname{deg}(E)=0$ on a compact Riemann surface $X$, which is generically regular semisimple. We have the family of harmonic bundles $\left(E, \bar{\partial}_{E}, t \theta, h_{t}\right)(t>0)$. Let $\nabla_{t}$ denote the Chern connection of $\left(E, \bar{\partial}_{E}, h_{t}\right)$. By Theorem 1.2, we can take a sub-sequence $t_{i} \rightarrow \infty$ such that the sequence of vector bundles with a Hermitian metric and a unitary connection $\left(E, \nabla_{t_{i}}, h_{t_{i}}\right)_{\mid X \backslash D(E, \theta)}$ converges in some sense to a vector bundle with a Hermitian metric and a unitary flat connection $\left(E_{\infty}, \nabla_{\infty}, h_{\infty}\right)$ on $X \backslash D(E, \theta)$. It is interesting to determine the flat connection $\nabla_{\infty}$. Following [11, 12], such a limit (or the associated parabolic bundle) is called a limiting configuration of the Higgs bundle $\left(E, \bar{\partial}_{E}, \theta\right)$ in this paper. Note that it is not clear, in general, whether $\nabla_{\infty}$ is independent of the choice of a sub-sequence. In this paper, we shall study the case rank $E=2$ by assuming that $\theta$ is generically regular semisimple, but without assuming that the zeroes of $\operatorname{det}(\theta)-(\operatorname{tr} \theta)^{2} / 4$ are simple. See $\S 1.2 .6$ for a remark on the case where the Higgs bundle is not generically regular semisimple.

Remark 1.6 The higher rank case has not yet been studied in general. Under some assumptions on the spectral curves, it looks possible to generalize the method in [11, 12] and our method in this paper. See also [3] for some Toda-like cases.

\subsubsection{The case where the spectral curve is smooth irreducible and simply ramified over $X$}

In $[11,12]$, Mazzeo, Swoboda, Weiss, and Witt studied the case under the assumption that rank $E=2$ and that the zeroes of $\operatorname{det}(\theta)-(\operatorname{tr} \theta)^{2} / 4$ are simple, i.e., the orders of the zeroes of the quadratic differential $\operatorname{det}(\theta)-(\operatorname{tr} \theta)^{2} / 4$ are at most 1, inspired by the work of Gaiotto, Moore and Neitzke [5]. (We remark that in $[11,12]$, the condition $\operatorname{deg}(E)=0$ is not imposed.) Let us describe the limiting configuration in this case. It is enough to consider the case $\operatorname{tr}(\theta)=0$. Under the assumption, the spectral curve $\widetilde{X}:=\Sigma(E, \theta)$ is smooth and connected. The natural projection $\pi: \widetilde{X} \longrightarrow X$ is the ramified covering of degree 2. The ramification index is at most 2. The discriminant $D(E, \theta)$ is exactly the set of the points on which $\pi$ is ramified. Let $\iota: \widetilde{X} \longrightarrow T^{*} X$ denote the inclusion. We have the line bundle $\widetilde{L}$ on $\widetilde{X}$ such that $M_{E, \theta} \simeq \iota_{*} \widetilde{L}$. We have $E \simeq \pi_{*} \widetilde{L}$.

We have the unique non-trivial involution $\rho: \widetilde{X} \longrightarrow \widetilde{X}$ over $X$, i.e., $\pi \circ \rho=\pi, \rho \circ \rho=\operatorname{id}_{\widetilde{X}}$ and $\rho \neq \operatorname{id}_{\widetilde{X}}$. We have the line bundle $\rho^{*} \widetilde{L}$ on $\widetilde{X}$. We have a natural inclusion of $\mathcal{O}_{\widetilde{X}^{-m o d u l e s}} \pi^{*} E \longrightarrow \widetilde{L} \oplus \rho^{*} \widetilde{L}$, and the cokernel is isomorphic to the structure sheaf of $\widetilde{D}(E, \theta):=\pi^{-1} D(E, \theta)$. We have $\widetilde{L} \otimes \rho^{*} \widetilde{L} \simeq \pi^{*} \operatorname{det}(E) \otimes \mathcal{O} \widetilde{X}(\widetilde{D}(E, \theta))$. Because $\operatorname{deg}(\widetilde{L})-|\widetilde{D}(E, \theta)| / 2=0$, we have a Hermitian metric $h_{\widetilde{L}}^{\lim }$ of $\widetilde{L}_{\mid \widetilde{X} \backslash \widetilde{D}(E, \theta)}$ with the following property. 
- The Chern connection of $\left(\widetilde{L}_{\mid \widetilde{X} \backslash \widetilde{D}(E, \theta)}, h_{\widetilde{L}}^{\lim }\right)$ is flat.

- Let $\widetilde{P} \in \widetilde{D}(E, \theta)$. Let $e_{\widetilde{P}}$ be a local frame of $\widetilde{L}$ around $\widetilde{P}$. Let $\left(\widetilde{U}_{\widetilde{P}}, w\right)$ be a holomorphic coordinate system around $\widetilde{P}$ with $w(\widetilde{P})=0$. Then, $\left|\log \left(|w| h_{\widetilde{L}}^{\lim }\left(e_{\widetilde{P}}, e_{\widetilde{P}}\right)\right)\right|$ is bounded on $\widetilde{U}_{\widetilde{P}} \backslash \widetilde{P}$.

- We have $h_{\widetilde{L}}^{\lim } \otimes \rho^{*} h_{\widetilde{L}}^{\lim }=\pi^{*} h_{\operatorname{det}(E)}$ on $\widetilde{X} \backslash \widetilde{D}(E, \theta)$.

Such $h_{\widetilde{L}}^{\lim }$ is uniquely determined. We have the induced Hermitian metric $h_{E, \theta}$ of $E_{\mid X \backslash D(E, \theta)}=\pi_{*}(\widetilde{L})_{\mid X \backslash D(E, \theta)}$. We have the Chern connection $\nabla_{E, \theta}$ of $\left(E_{\mid X \backslash D(E, \theta)}, h_{E, \theta}^{\lim }\right)$. This is the limiting configuration of $\left(E, \bar{\partial}_{E}, \theta\right)$ in this case. Interestingly, in $[11,12]$, it is proved that the family $\left(E, \bar{\partial}_{E}, h_{t}, \theta\right)_{\mid X \backslash D(E, \theta)}(t>0)$ is convergent to $\left(E_{\mid X \backslash D(E, \theta)}, \bar{\partial}_{E}, h_{E, \theta}^{\lim }, \theta\right)$ in an appropriate sense.

\subsubsection{Limiting configuration in the general case}

It is natural to study the case where $\theta$ is generically regular semisimple, but the zeroes of $\operatorname{det}(\theta)-(\operatorname{tr} \theta)^{2} / 4 \operatorname{are}$ not necessarily simple. We may assume $\operatorname{tr} \theta=0$. It is enough to consider the case where the spectral curve of the Higgs bundle $\left(E, \bar{\partial}_{E}, \theta\right)$ is reducible to the two components, i.e., we have holomorphic 1-forms $\omega \neq 0$ such that $\Sigma(E, \theta)=\operatorname{Im}(\omega) \cup \operatorname{Im}(-\omega)$. Indeed, if the spectral curve is irreducible, we have only to consider the pull back of the Higgs bundle by a ramified covering map of degree 2 given by the normalization of the spectral curve. (See $\S 1.2 .5$. See $\S 4.3 .2$ and $\S 5.1 .3$ for more details.) Let $Z(\omega)$ denote the zero set of $\omega$, which is equal to the discriminant $D(E, \theta)$.

We have two line bundles $L_{i}(i=1,2)$ with an inclusion $\iota_{E}: E \longrightarrow L_{1} \oplus L_{2}$ with the following property:

- Set $\theta_{L_{1} \oplus L_{2}}:=\omega \operatorname{id}_{L_{1}} \oplus(-\omega) \operatorname{id}_{L_{2}}$. Then, we have $\theta_{L_{1} \oplus L_{2}} \circ \iota_{E}=\iota_{E} \circ \theta$.

- The restriction $\iota_{E \mid X \backslash Z(\omega)}$ is an isomorphism.

- The induced morphisms $E \longrightarrow L_{i}$ are surjective.

Set $d_{i}:=\operatorname{deg}\left(L_{i}\right)$. We may assume that $d_{1} \leq d_{2}$. For each $P \in Z(\omega)$, let $m_{P}$ denote the order of the zero of $\omega$ at $P$. Namely, for a holomorphic coordinate system $\left(U_{P}, z\right)$ around $P$ with $z(P)=0$, we have $\omega_{\mid U_{P}}=g_{P} \cdot z^{m_{P}} d z$ with $g_{P}(P) \neq 0$. The support of the cokernel $\left(L_{1} \oplus L_{2}\right) / E$ is contained in $Z(\omega)$. For each $P \in Z(\omega)$, let $\ell_{P}$ denote the length of the stalk of $\left(L_{1} \oplus L_{2}\right) / E$ at $P$. Note that we have $L_{1} \otimes L_{2}=\operatorname{det}(E) \otimes \mathcal{O}\left(\sum_{P \in Z(\omega)} \ell_{P} P\right)$. In particular, we have

$$
d_{1}+d_{2}-\sum_{P \in Z(\omega)} \ell_{P}=\operatorname{deg}(E)=0 .
$$

Let $L_{1}^{\prime}$ denote the kernel of $E \longrightarrow L_{2}$. Similarly, let $L_{2}^{\prime}$ denote the kernel of $E \longrightarrow L_{1}$. Proper subbundles of $E$ preserved by $\theta$ are only $L_{1}^{\prime}$ and $L_{2}^{\prime}$. Hence, the stability condition for $(E, \theta)$ is equivalent to $\operatorname{deg}\left(L_{i}^{\prime}\right)<0$ $(i=1,2)$. It is equivalent to $d_{i}=\operatorname{deg}\left(L_{i}\right)>0(i=1,2)$.

To give the limiting configuration of $\left(E, \bar{\partial}_{E}, \theta\right)$, we would like to give "parabolic weights" of $L_{i}(i=1,2)$ at $Z(\omega)$. In $\$ 1.2 .2$, all the parabolic weights are $1 / 2$. In the general case, it turns out that the parabolic weights are given by the following rule.

For each $P \in Z(\omega)$, we consider the piecewise linear function $\chi_{P}: \mathbb{R}_{\geq 0} \longrightarrow \mathbb{R}_{\leq 0}$ given by

$$
\chi_{P}(a):= \begin{cases}\left(m_{P}+1\right) a-\ell_{P} / 2 & \left(a \leq \ell_{P} / 2\left(m_{P}+1\right)\right) \\ 0 & \left(a>\ell_{P} / 2\left(m_{P}+1\right)\right)\end{cases}
$$

We set $\chi_{E, \theta}(a):=\sum_{P \in Z(\omega)} \chi_{P}(a)$. Then, we have a unique $0 \leq a_{E, \theta}<\max _{P \in Z(\omega)}\left\{\ell_{P} / 2\left(m_{P}+1\right)\right\}$ such that $d_{1}+\chi_{E, \theta}\left(a_{E, \theta}\right)=0$.

We determine the parabolic weights of $L_{1}$ at $P \in Z(\omega)$ as $-\chi_{P}\left(a_{E, \theta}\right)$, and the parabolic weights of $L_{2}$ at $P \in Z(\omega)$ as $\chi_{P}\left(a_{E, \theta}\right)+\ell_{P}$. We have associated singular Hermitian metrics on $L_{i}$, i.e., we consider Hermitian metrics $h_{L_{i}}^{\lim }(i=1,2)$ on $L_{i \mid X \backslash Z(\omega)}$ satisfying the following conditions.

- The Chern connections of $\left(L_{i \mid X \backslash Z(\omega)}, h_{L_{i}}^{\lim }\right)$ are flat. 
- Let $P \in Z(\omega)$. Let $v_{P, i}$ be local frames of $L_{i}$ around $P$. Let $\left(U_{P}, z\right)$ be a holomorphic coordinate neighbourhood around $P$ with $z(P)=0$. Then, the functions

$$
\log \left(|z|^{-2 \chi_{P}\left(a_{E, \theta}\right)} h_{L_{1}}^{\lim }\left(v_{P, 1}, v_{P, 1}\right)\right), \quad \log \left(|z|^{2 \chi_{P}\left(a_{E, \theta}\right)+2 \ell_{P}} h_{L_{2}}^{\lim }\left(v_{P, 2}, v_{P, 2}\right)\right)
$$

are bounded on $U_{P} \backslash P$.

- We have $h_{L_{1}}^{\lim } \otimes h_{L_{2}}^{\lim }=h_{\operatorname{det}(E)}$.

We obtain the Hermitian metric $h_{E, \theta}^{\lim }=h_{L_{1}}^{\lim } \oplus h_{L_{2}}^{\lim }$ of $E_{\mid X \backslash Z(\omega)}$, and the Chern connection $\nabla_{E, \theta}^{\lim }$.

Remark 1.7 We still have the ambiguity for $h_{L_{i}}^{\lim }$. Namely, if $h_{L_{i}}^{\lim }(i=1,2)$ satisfy the above conditions, $\alpha h_{L_{1}}^{\lim }$ and $\alpha^{-1} h_{L_{2}}^{\lim }$ also satisfy the above conditions for any $\alpha>0$. In $\S 1.2 .2$, we have an additional condition $\rho^{*} h_{L_{1}}^{\lim }=h_{L_{2}}^{\lim }$ under the natural isomorphism $\rho^{*} L_{1} \simeq L_{2}$, with which the metrics are uniquely determined. But, in general, it seems that we do not have such an extra condition.

The metric $h_{E, \theta} \lim _{\text {is }}$ also characterized as a harmonic metric of the polystable Higgs bundle $\left(E, \bar{\partial}_{E}, \theta\right)_{\mid X \backslash Z(\omega)}$ adapted to the parabolic structure given as above.

Although we have the ambiguity of the metrics, the connection $\nabla_{E, \theta}^{\lim }$ is uniquely determined.

Remark 1.8 Let us consider the case where $m_{P}=\ell_{P}=1$ for any $P \in Z(\omega)$, and $d_{1}=d_{2}=|Z(\omega)| / 2$. Then, we have $a_{E, \theta}=0$, and hence $-\chi_{P}\left(a_{E, \theta}\right)=\chi_{P}\left(a_{E, \theta}\right)+\ell_{P}=1 / 2$. These are the parabolic weights appeared in $\S 1.2 .2$.

Remark 1.9 It might be instructive to mention that if we have $\operatorname{deg}\left(L_{1}\right)=\operatorname{deg}\left(L_{2}\right)$ then we have $a_{E, \theta}=0$, and $-\chi_{P}\left(a_{E, \theta}\right)=\chi_{P}\left(a_{E, \theta}\right)+\ell_{P}=\ell_{P} / 2$.

\subsubsection{Convergence to the limiting configuration}

Suppose that $\left(E, \bar{\partial}_{E}, \theta\right)$ is stable of degree 0 with rank $E=2$ and that $\theta$ is generically regular semisimple. For simplicity, we assume $\operatorname{tr} \theta=0$. Let $h_{t}(t>0)$ denote the harmonic metric of the Higgs bundle $\left(E, \bar{\partial}_{E}, t \theta\right)$ with $\operatorname{det}\left(h_{t}\right)=h_{\operatorname{det}(E)}$.

For any $\alpha>0$, let $\Psi_{\alpha}$ be the automorphism of $L_{1} \oplus L_{2}$ given by $\Psi_{\alpha}=\alpha \operatorname{id}_{L_{1}} \oplus \alpha^{-1} \operatorname{id}_{L_{2}}$. Let $\Psi_{\alpha}^{*} h_{t}$ be the metric of $E_{\mid X \backslash D(E, \theta)}$ given by $h_{t}\left(\Psi_{\alpha} s_{1}, \Psi_{\alpha} s_{2}\right)$ for local sections $s_{j}(j=1,2)$ of $E_{\mid X \backslash D(E, \theta)}$. We take any point $Q \in X \backslash D(E, \theta)$ and a frame $v_{Q}$ of $L_{1 \mid Q}$, and we put

$$
\gamma(t, Q):=\left(\frac{h_{L_{1}}^{\lim }\left(v_{Q}, v_{Q}\right)}{h_{t}\left(v_{Q}, v_{Q}\right)}\right)^{1 / 2} .
$$

The following theorem is our second main result in this paper.

Theorem 1.10 (The degree 0 case of Theorem 5.1) When $t$ goes to $\infty$, the sequence $\Psi_{\gamma(t, Q)}^{*} h_{t}$ converges to $h_{E, \theta} \operatorname{lin}_{\text {in }} C^{\infty}$-sense on any compact subset in $X \backslash Z(\omega)$. In particular, the sequence of the connections $\nabla_{t}$ converges to $\nabla_{E, \theta} \lim _{\text {. }}\left(\right.$ See $§ 1.2 .3$ for $h_{E, \theta}^{\lim _{1}}$ and $\nabla_{E, \theta}^{\lim }$.)

For the proof of Theorem 5.1, we need to study the global property of $\left(E, \bar{\partial}_{E}, \theta\right)$, in contrast that we can obtain the estimates in $\S 1.1 .2$ locally at any point of $X \backslash D(E, \theta)$. A key is the construction of a family of Hermitian metrics $h_{t}^{0}$ of $E$ such that the $L^{p}$-norms of $R\left(h_{t}^{0}\right)+\left[t \theta,(t \theta)_{h_{t}^{0}}^{\dagger}\right]$ are bounded, for which we naturally encounter the above parabolic weights for the limiting configuration. We have the family of the self-adjoint endomorphisms $k_{t}$ of $\left(E, h_{t}^{0}\right)$ such that $h_{t}\left(u_{1}, u_{2}\right)=h_{t}^{0}\left(k_{t} u_{1}, u_{2}\right)$ for any local sections $u_{i}(i=1,2)$. By applying a variant of the arguments used in [15] with the tools given in [19], we shall observe that the sequence $\kappa_{i} k_{t_{i}}$ converges in some sense for an appropriate sequence $\kappa_{i}>0$. Then, the claim of the theorem follows.

We note that for the construction of the family of metrics $h_{t}^{0}$, we use the general theory of wild harmonic bundles. We need a family of harmonic metrics given on a neighbourhood of the discriminant locus, for which we apply the Kobayashi-Hitchin correspondence for wild harmonic bundles on curves given in [2] (see [20] for 
the tame case). On the basis of the general results on the asymptotic behaviour of wild harmonic bundles studied in [20] and [16], we can deduce rather detailed properties of the family of the harmonic metrics as in Proposition 3.17 and Proposition 3.19.

At this moment, it is not clear to the author whether we could directly use the argument in [11] to prove Theorem 1.10 or its variant in our setting. But, we should note that we do not study the order of the convergence in Theorem 1.10. In contrast, when all the zeroes of $\operatorname{det} \theta-(\operatorname{tr} \theta)^{2} / 4$ are simple, the method in [11] is strong enough to give the order of the convergence.

Remark 1.11 Let $\left(E, \bar{\partial}_{E}, \theta\right)$ be a stable Higgs bundle of rank 2 on $X$ such that $\theta$ is generically regular semisimple, but that $\operatorname{deg}(E)$ is not necessarily 0 . We fix a Hermitian metric $h_{\operatorname{det}(E)}$ of $\operatorname{det}(E)$. For each $t>0$, we have the Hermitian-Einstein metric $h_{t}^{H E}$ of $\left(E, \bar{\partial}_{E}, \theta\right)$ such that $\operatorname{det}\left(h_{t}^{H E}\right)=h_{\operatorname{det} E}$, according to [6] and [19]. Here, the Hermitian-Einstein condition means the trace-free part of $R\left(h_{t}^{H E}\right)+\left[t \theta,(t \theta)_{h_{t}^{H E}}^{\dagger}\right]$ is 0 . We can study the behaviour of $h_{t}^{H E}(t \rightarrow \infty)$ by using Theorem 1.10. (See Theorem 5.1.)

Suppose that $\operatorname{deg}(E)=2 m$ for an integer $m$. We take a line bundle $L$ with $\operatorname{deg}(L)=-m$. We can easily reduce the study on the behaviour of $h_{t}^{H E}$ to the study on the behaviour of harmonic metrics for $\left(E, \bar{\partial}_{E}, \theta\right) \otimes L$. Suppose that $\operatorname{deg}(E)$ is odd. We take any (unramified) covering $\operatorname{map} \varphi: X^{\prime} \longrightarrow X$ of degree 2 . Then, the degree of $\varphi^{*}\left(E, \bar{\partial}_{E}, \theta\right)$ is even. Hence, the study can be reduced to the degree 0 case. (See also §5.2.)

\subsubsection{Symmetric case and irreducible case}

Let us explain that we can obtain a stronger result if the Higgs bundle is equipped with an extra symmetry. Suppose that $X$ is equipped with a non-trivial involution $\rho$, i.e., $\rho: X \longrightarrow X$ is a holomorphic automorphism such that $\rho \circ \rho=\operatorname{id}_{X}$ and $\rho \neq \operatorname{id}_{X}$. Let $\left(E, \bar{\partial}_{E}, \theta\right)$ be a stable Higgs bundle of degree 0 on $X$ with $\operatorname{tr} \theta=0$ such that the spectral curve is reducible, i.e., $\Sigma(E, \theta)=\operatorname{Im}(\omega) \cup \operatorname{Im}(-\omega)$ for a holomorphic one form $\omega \neq 0$ on $X$. We impose the following conditions.

- We have $\rho^{*} \omega=-\omega$.

- The Higgs bundle $\left(E, \bar{\partial}_{E}, \theta\right)$ is equivariant with respect to the action of $\{1, \rho\}$ on $X$.

We have the induced isomorphism $\rho^{*} \operatorname{det}(E) \simeq \operatorname{det}(E)$. We naturally have $\rho^{*} h_{\operatorname{det}(E)}=h_{\operatorname{det}(E)}$.

Let $L_{i}(i=1,2)$ be as in $\S 1.2 .3$. We naturally have the isomorphisms $\rho^{*} L_{1} \simeq L_{2}$ and $\rho^{*} L_{2} \simeq L_{1}$, which are compatible with the isomorphism $\rho^{*} E \simeq E$. Because $\operatorname{deg}\left(L_{1}\right)=\operatorname{deg}\left(L_{2}\right)$, we have $a_{E, \theta}=0$ and $-\chi_{P}\left(a_{E, \theta}\right)=$ $\chi_{P}\left(a_{E, \theta}\right)+\ell_{P}=\ell_{P} / 2$ for any $P \in Z(\omega)$. We can uniquely determine the metrics $h_{L_{j}}^{\lim }$ by imposing the extra condition $\rho^{*} h_{L_{1}}^{\lim }=h_{L_{2}}^{\lim }$.

Theorem 1.12 (The degree 0 case of Theorem 5.3) For $t>0$, let $h_{t}$ be the harmonic metrics of the Higgs bundles $\left(E, \bar{\partial}_{E}, t \theta\right)$ such that $\operatorname{det}\left(h_{t}\right)=h_{\operatorname{det}(E)}$. Then, the sequence $h_{t}$ is convergent to $h_{E, \theta}^{\lim _{1}}=h_{L_{1}}^{\lim } \oplus h_{L_{2}}^{\lim }$ on any compact subset in $X \backslash Z(\omega)$.

Let us remark that we can apply Theorem 1.12 to the case where the spectral curve is irreducible and the Higgs field is generically regular semisimple. Let $X^{\prime}$ be a compact connected Riemann surface. Let $\left(E^{\prime}, \bar{\partial}_{E^{\prime}}, \theta^{\prime}\right)$ be a stable Higgs bundle of rank 2 with $\operatorname{deg}\left(E^{\prime}\right)=0$ on $X^{\prime}$ such that (i) the spectral curve $\Sigma\left(E^{\prime}, \theta^{\prime}\right)$ is irreducible, (ii) $\operatorname{tr} \theta^{\prime}=0$, (iii) $\theta^{\prime}$ is generically regular semisimple. We take the normalization $\kappa: X \longrightarrow \Sigma\left(E^{\prime}, \theta^{\prime}\right)$. Let $p: X \longrightarrow X^{\prime}$ be the morphism obtained as the composite of $\kappa$ and the projection $\Sigma\left(E^{\prime}, \theta^{\prime}\right) \longrightarrow X^{\prime}$. We set $\left(E, \bar{\partial}_{E}, \theta\right)=p^{*}\left(E^{\prime}, \bar{\partial}_{E^{\prime}}, \theta^{\prime}\right)$. We have the involution on $\Sigma\left(E^{\prime}, \theta^{\prime}\right)$ induced by the multiplication of -1 on the cotangent bundle $T^{*} X^{\prime}$. It induces an involution $\rho$ on $X$. We fix a Hermitian metric $h_{\operatorname{det}\left(E^{\prime}\right)}$ of $\operatorname{det}\left(E^{\prime}\right)$.

If $\left(E, \bar{\partial}_{E}, \theta\right)$ is polystable, we can easily observe that the harmonic metrics $h_{t}^{\prime}$ for the Higgs bundles $\left(E^{\prime}, \bar{\partial}_{E^{\prime}}, t \theta^{\prime}\right)$ are independent of $t$. (See $\S 4.3 .2$.) We formally set $h_{E^{\prime}, \theta^{\prime}}^{\lim }:=h_{t}^{\prime}$.

If $\left(E, \bar{\partial}_{E}, \theta\right)$ is stable, the above symmetry conditions for $\left(E, \bar{\partial}_{E}, \theta\right)$ and $\rho$ are satisfied. The pull back $h_{\operatorname{det}(E)}=p^{*} h_{\operatorname{det}\left(E^{\prime}\right)}$ satisfies the condition $\rho^{*} h_{\operatorname{det}(E)}=h_{\operatorname{det}(E)}$. We have the metrics $h_{L_{i}}^{\lim }$ and $h_{E, \theta}^{\lim }=h_{L_{1}}^{\lim } \oplus h_{L_{2}}^{\lim }$ as above. Because we have $\rho^{*} h_{E, \theta}=h_{E, \theta}$, we have a unique Hermitian metric $h_{E^{\prime}, \theta^{\prime}}$ on $X^{\prime} \backslash D\left(E^{\prime}, \theta^{\prime}\right)$ such that $p^{*} h_{E^{\prime}, \theta^{\prime}}=h_{E, \theta}$. We also have a characterization of $h_{E^{\prime}, \theta^{\prime}}^{\lim }$ as a harmonic metric for the stable filtered 
Higgs bundles on $\left(X^{\prime}, D\left(E^{\prime}, \theta^{\prime}\right)\right)$ induced by the limiting configuration of $\left(E, \bar{\partial}_{E}, \theta\right)$. (See $\S 4.3 .2$.) We have the following direct corollary.

Corollary 1.13 (The degree 0 case of Corollary 5.2) For $t>0$, let $h_{t}^{\prime}$ be the harmonic metrics of the

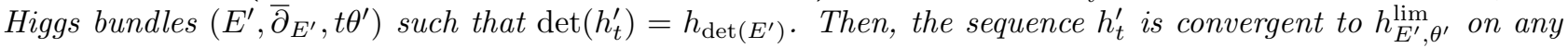
compact subset in $X^{\prime} \backslash D\left(E^{\prime}, \theta^{\prime}\right)$.

\subsubsection{Complement}

Let $\left(E, \bar{\partial}_{E}, \theta, h\right)$ be a Higgs bundle of rank 2. Suppose that $\theta$ is nilpotent, i.e., $\Sigma(E, \theta)$ is the 0 -section in $T^{*} X$. Then, we can easily observe that $\lim _{t \rightarrow \infty}\left(E, \bar{\partial}_{E}, t \theta, h_{t}\right)$ is convergent to a polarized complex variation of Hodge structure. We sketch it. We have a polystable Higgs bundle $\left(E_{\infty}, \bar{\partial}_{\infty}, \theta_{\infty}\right)$ obtained as the limit $\lim _{t \rightarrow \infty}\left(E, \bar{\partial}_{E}, t \theta\right)$ in the coarse moduli space of semistable Higgs bundles of degree 0 on $X$. Note that the norms $\left|R\left(\nabla_{h_{t}}\right)\right|_{h_{t}, g_{X}}=|t \theta|_{h_{t}, g_{X}}^{2}(t>0)$ are uniformly bounded, where $g_{X}$ is a Kähler metric of $X$. (See Proposition 2.1, for example.) Hence, we obtain a harmonic metric $h_{\infty}$ of $\left(E_{\infty}, \bar{\partial}_{\infty}, \theta_{\infty}\right)$ as the limit of a convergent subsequence $h_{t_{i}}\left(t_{i} \rightarrow \infty\right)$. If $\left(E_{\infty}, \bar{\partial}_{\infty}, \theta_{\infty}\right)$ is not stable, $\theta_{\infty}$ is trivial, $\left(E_{\infty}, \bar{\partial}_{\infty}\right)$ is a direct sum of line bundles, and $h_{\infty}$ is a flat metric. If $\left(E_{\infty}, \bar{\partial}_{\infty}, \theta_{\infty}\right)$ is stable, then as in the case of the limit for $t \rightarrow 0$ studied by Simpson, we can observe that $\left(E_{\infty}, \bar{\partial}_{\infty}, \theta_{\infty}\right)$ is a Hodge bundle, and $h_{\infty}$ is equivariant with respect to the natural grading $S^{1}$-action. Hence, $\left(E_{\infty}, \bar{\partial}_{\infty}, \theta_{\infty}, h_{\infty}\right)$ comes from a polarized complex variation of Hodge structure.

In view of the moduli theoretic picture $[6,22]$, we can see it as follows. The energies of the family of $\left(E, \bar{\partial}_{E}, t \theta, h_{t}\right)(t>0)$ are bounded. So, the family is relatively compact in the moduli space of harmonic bundles. Hence, when $t$ goes to $\infty,\left(E, \bar{\partial}_{E}, \theta, h_{t}\right)$ goes to a fixed point in the moduli space induced by the natural $\mathbb{C}^{*}$-action on the moduli space of Higgs bundles. It means that $\left(E, \bar{\partial}_{E}, \theta, h_{t}\right)$ is convergent to a polarized complex variation of Hodge structure.

If $\left(E, \bar{\partial}_{E}, \theta\right)$ is not generically regular semisimple but $\operatorname{rank} E=2$, then the study of $\lim _{t \rightarrow \infty}\left(E, \bar{\partial}_{E}, t \theta, h_{t}\right)$ is easily reduced to the above case.

\subsection{Acknowledgement}

The author thanks the referee for his thorough reading and valuable remarks. This study has grown out of my effort to understand (part of) the intriguing works [3], [5], [7] and [11, 12]. I thank Carlos Simpson for inspiring discussions on many occasions. Indeed, I am motivated to this study by his question several years ago. I owe a lot to his philosophy and his techniques. I also thank him for many valuable comments to improve this manuscript, and for his patience in reading the preliminary versions of this manuscript. I hope that this study would be useful for the project in [7]. I learned the attractive study $[11,12]$ from the stimulating talk given by Frederik Witt in the conference "The Geometry, Topology and Physics of Moduli Spaces of Higgs Bundles" held at the Institute for Mathematical Sciences, National University of Singapore in 2014, for which the visit was supported by the Institute. I thank Claude Sabbah for his kindness and for many discussions. I am grateful to Yoshifumi Tsuchimoto and Akira Ishii for their constant encouragement. I thank Szilard Szabo for his interest to this study and some discussions.

This work was partially supported by the Grant-in-Aid for Scientific Research (S) (No. 24224001) and the Grant-in-Aid for Scientific Research (C) (No. 15K04843), Japan Society for the Promotion of Science.

\section{Asymptotic decoupling}

\subsection{Simpson's main estimate for harmonic bundles on discs}

Let $\left(E, \bar{\partial}_{E}, \theta, h\right)$ be a harmonic bundle on a complex curve $X$. We have the spectral curve $\Sigma(E, \theta)$ in the cotangent bundle of $X$. We have an estimate of the norm of $\theta$, depending only on $\Sigma(E, \theta)$ (Proposition 2.10). If $\Sigma(E, \theta)$ is decomposed into a disjoint union $\Sigma_{1} \sqcup \Sigma_{2}$, then we have the corresponding decomposition of the Higgs bundle $\left(E, \bar{\partial}_{E}, \theta\right)=\left(E_{1}, \bar{\partial}_{E_{1}}, \theta_{1}\right) \oplus\left(E_{2}, \bar{\partial}_{E_{2}}, \theta_{2}\right)$. The Hermitian product of sections of $E_{1}$ and $E_{2}$ given by $h$ should be small. We have such estimates depending only on $\Sigma(E, \theta)$ (Corollary 2.6). Because we use the 
arguments essentially given in [20], such estimates are called Simpson's main estimate. It is enough to consider the case where $X$ is a disc.

\subsubsection{Estimate of the sup norm in terms of the eigenvalues}

For $R>0$, we set $\Delta(R):=\{z \in \mathbb{C}|| z \mid<R\}$. We consider a harmonic bundle $\left(E, \bar{\partial}_{E}, \theta, h\right)$ of rank $r$ on $\Delta(R)$. We have the description $\theta=f d z$, where $f$ is a holomorphic endomorphism of $E$. Fix $M>0$, and suppose the following.

- For any $P \in \Delta(R)$, the eigenvalues $\gamma$ of $f_{\mid P}$ satisfy $|\gamma|<M$.

We recall the following proposition, for which a proof was given in [14], for example. We include an outline of the proof for the convenience of the readers.

Proposition 2.1 Fix $0<R_{1}<R$. Then, we have $C_{1}, C_{2}>0$ depending only on $r, R_{1}, R$ such that

$$
|f|_{h} \leq C_{1} M+C_{2}
$$

holds on $\Delta\left(R_{1}\right)$.

Proof Let $f_{h}^{\dagger}$ denote the adjoint of $f$ with respect to $h$. As in [20], we have the following inequality on $\Delta(R)$ (see also Lemma 2.4 below):

$$
-\partial_{z} \partial_{\bar{z}} \log |f|_{h}^{2} \leq-\frac{\left|\left[f, f_{h}^{\dagger}\right]\right|_{h}^{2}}{|f|_{h}^{2}}
$$

For any $P \in \Delta(R)$, we set $g(P):=\sum_{i=1}^{r}\left|\alpha_{i}\right|^{2}$, where $\alpha_{1}, \ldots, \alpha_{r}$ are the eigenvalues of $f_{\mid P}$. There exists a constant $C_{3}>0$ depending only on $r$ such that

$$
\left|\left[f, f_{h}^{\dagger}\right]_{\mid P}\right|_{h} \geq C_{3}\left(\left|f_{\mid P}\right|_{h}^{2}-g(P)\right) .
$$

We obtain

$$
-\partial_{z} \partial_{\bar{z}} \log |f|_{h}^{2}(P) \leq-C_{3}^{2} \frac{\left.|| f_{\mid P}\right|_{h} ^{2}-\left.g(P)\right|^{2}}{\left|f_{\mid P}\right|_{h}^{2}}
$$

Note that if $\left|f_{\mid P}\right|_{h}^{2} \geq 2 g(P)$ for some $P$, then we have

$$
-\frac{\left.|| f_{\mid P}\right|_{h} ^{2}-\left.g(P)\right|^{2}}{\left|f_{\mid P}\right|_{h}^{2}} \leq-\frac{1}{4}\left|f_{\mid P}\right|_{h}^{2} .
$$

For any positive number $B$, we have

$$
-\partial_{z} \partial_{\bar{z}} \log \frac{B}{\left(R^{2}-|z|^{2}\right)^{2}}=-\frac{2 R^{2}}{B} \frac{B}{\left(R^{2}-|z|^{2}\right)^{2}} .
$$

Take $B$ satisfying $B \geq 8 R^{2} / C_{3}^{2}$ and $B \geq 2 R^{4} r M^{2}$. Then, we use an idea in the proof of a lemma of Ahlfors [1]. Set $\mathcal{Z}:=\left\{\left.P \in \Delta(R)|| f_{\mid P}\right|_{h} ^{2}>B\left(R^{2}-|z(P)|^{2}\right)^{-2}\right\}$. Suppose that $\mathcal{Z} \neq \emptyset$, and we shall derive a contradiction. We have $\left|f_{\mid P}\right|_{h}^{2}>B R^{-4} \geq 2 r M^{2}>2 g(P)$ for any $P \in \mathcal{Z}$. Hence, the following inequality holds on $\mathcal{Z}$ :

$$
-\partial_{z} \partial_{\bar{z}}\left(\log |f|_{h}^{2}-\log \left(B\left(R^{2}-|z|^{2}\right)^{-2}\right)\right) \leq-\frac{C_{3}^{2}}{4}\left(|f|_{h}^{2}-B\left(R^{2}-|z|^{2}\right)^{-2}\right) \leq 0
$$

Note that $\mathcal{Z}$ is relatively compact in $\Delta(R)$. So, we have $|f|_{h}^{2}=B\left(R^{2}-|z|^{2}\right)^{-2}$ on the boundary of $\mathcal{Z}$, and hence we obtain $|f|_{h}^{2} \leq B\left(R^{2}-|z|^{2}\right)^{-2}$ on $\mathcal{Z}$. But, it contradicts with the choice of $\mathcal{Z}$. So, we obtain $\mathcal{Z}=\emptyset$. Namely, we have $|f|_{h}^{2} \leq B\left(R^{2}-|z|^{2}\right)^{-2}$ on $\Delta(R)$. Then, we obtain the claim of the proposition. 


\subsubsection{Asymptotic orthogonality}

We continue to use the notation in $\S 2.1 .1$. Suppose that we have a finite subset $S \subset \mathbb{C}$ and a decomposition $(E, \theta)=\bigoplus_{\alpha \in S}\left(E_{\alpha}, \theta_{\alpha}\right)$. We have the expression $\theta_{\alpha}=f_{\alpha} d z$ for each $\alpha$, where $f_{\alpha}$ is a holomorphic endomorphism of $E_{\alpha}$.

Assumption 2.2 Fix $C_{10}>1$, and we impose the following conditions.

- We set $d:=\min \{|\alpha-\beta| \mid \alpha, \beta \in S, \alpha \neq \beta\}$. Then, we have $d \geq 1$ and $M \leq C_{10} d$. Here, $M$ is the constant in the beginning of $\S 2.1 .1$.

- For any $P \in \Delta(R)$, the eigenvalues $\gamma$ of $f_{\alpha \mid P}$ satisfy $|\gamma-\alpha| \leq d / 100$.

Let $\pi_{\alpha}$ be the projection of $E$ onto $E_{\alpha}$ with respect to the above decomposition. Let $\pi_{\alpha}^{\prime}$ denote the orthogonal projection of $E$ onto $E_{\alpha}$. We set $\rho_{\alpha}:=\pi_{\alpha}-\pi_{\alpha}^{\prime}$. The following proposition is a variant of the estimates given in $[20]$ and $[13,16]$ for harmonic bundles given on punctured discs or the products of punctured discs, but it is more useful for the purpose in this paper.

Proposition 2.3 Let $R_{1}$ be as in Proposition 2.1. Fix $0<R_{2}<R_{1}$. There exist positive constants $\epsilon_{0}$ and $C_{11}$ depending only on $R, R_{1}, R_{2}, r$ and $C_{10}$, such that $\left|\rho_{\alpha}\right|_{h} \leq C_{11} \exp \left(-\epsilon_{0} d\right)$ on $\Delta\left(R_{2}\right)$.

Proof We recall a general inequality for holomorphic sections of $\operatorname{End}(E)$ which commutes with $\theta$, for the convenience of the readers.

Lemma 2.4 Let $s$ be any holomorphic section of $\operatorname{End}(E)$ such that $[\theta, s]=0$. Then, we have the following inequality:

$$
-\partial_{z} \partial_{\bar{z}} \log |s|_{h}^{2} \leq-\frac{\left|\left[f_{h}^{\dagger}, s\right]\right|_{h}^{2}}{|s|_{h}^{2}}
$$

Proof We have the equality $-\partial_{z} \partial_{\bar{z}}|s|_{h}^{2}=-\left|\partial_{z, h} s\right|_{h}^{2}-h\left(s, \partial_{\bar{z}} \partial_{z, h} s\right)$. Hence, we obtain the following:

$$
\begin{array}{r}
-\partial_{z} \partial_{\bar{z}} \log |s|_{h}^{2}=-\frac{\partial_{z} \partial_{\bar{z}}|s|_{h}^{2}}{|s|_{h}^{2}}+\frac{\partial_{z}|s|_{h}^{2}}{|s|_{h}^{2}} \frac{\partial_{\bar{z}}|s|_{h}^{2}}{|s|_{h}^{2}}=-\frac{h\left(s, \partial_{\bar{z}} \partial_{z, h} s\right)}{|s|_{h}^{2}}-\frac{\left|\partial_{z, h} s\right|_{h}^{2}}{|s|_{h}^{2}}+\frac{h\left(\partial_{z, h} s, s\right)}{|s|_{h}^{2}} \frac{h\left(z, \partial_{z, h} s\right)}{|s|_{h}^{2}} \\
\leq-\frac{h\left(s, \partial_{\bar{z}} \partial_{z, h} s\right)}{|s|_{h}^{2}}=-\frac{h\left(s,\left(\partial_{\bar{z}} \partial_{z, h}-\partial_{z, h} \partial_{\bar{z}}\right) s\right)}{|s|_{h}^{2}} .
\end{array}
$$

By the Hitchin equation, we have $R(h)+\left[\theta, \theta_{h}^{\dagger}\right]=R(h)+\left[f, f_{h}^{\dagger}\right] d z d \bar{z}=0$, i.e., $R(h)=\left[f, f_{h}^{\dagger}\right] d \bar{z} d z$. We obtain the following from (2):

$$
-\partial_{z} \partial_{\bar{z}} \log |s|_{h}^{2} \leq-\frac{h\left(s,\left[\left[f, f_{h}^{\dagger}\right], s\right]\right)}{|s|_{h}^{2}}
$$

Because $[f, s]=0$, we obtain

$$
-\partial_{z} \partial_{\bar{z}} \log |s|_{h}^{2} \leq-\frac{h\left(s,\left[f,\left[f_{h}^{\dagger}, s\right]\right]\right)}{|s|_{h}^{2}}=-\frac{h\left(\left[f_{h}^{\dagger}, s\right],\left[f_{h}^{\dagger}, s\right]\right)}{|s|_{h}^{2}}=-\frac{\left|\left[f_{h}^{\dagger}, s\right]\right|_{h}^{2}}{|s|_{h}^{2}}
$$

Thus, we obtain Lemma 2.4 .

Let $r_{\alpha}:=\operatorname{rank} E_{\alpha}$. Because $\partial_{\bar{z}} \partial_{z} \log r_{\alpha}=0$, we obtain the following inequality on $\Delta(R)$ from (1):

$$
-\partial_{z} \partial_{\bar{z}} \log \left(\left|\pi_{\alpha}\right|_{h}^{2} / r_{\alpha}\right) \leq-\frac{\left|\left[f_{h}^{\dagger}, \pi_{\alpha}\right]\right|_{h}^{2}}{\left|\pi_{\alpha}\right|_{h}^{2}}
$$

According to Proposition 2.1 and Lemma 2.7 below, there exists a positive constant $C_{12}$ depending only on $R, R_{1}, r$ and $C_{10}$ such that we have $\left|\pi_{\alpha}\right|_{h} \leq C_{12}$ and $\left|\rho_{\alpha}\right|_{h} \leq C_{12}$ on $\Delta\left(R_{1}\right)$. We set $k_{\alpha}:=\log \left(\left|\pi_{\alpha}\right|_{h}^{2} / r_{\alpha}\right)$. Note 
that $\left|\pi_{\alpha}\right|_{h}^{2}=\left|\pi_{\alpha}^{\prime}\right|_{h}^{2}+\left|\rho_{\alpha}\right|_{h}^{2}$ and $\left|\pi_{\alpha}^{\prime}\right|_{h}^{2}=r_{\alpha}$. Hence, we have $k_{\alpha}=\log \left(1+\left|\rho_{\alpha}\right|^{2} / r_{\alpha}\right)$. There exists a positive constant $C_{13}$ depending only on $R, R_{1}, r$ and $C_{10}$ such that $C_{13}^{-1}\left|\rho_{\alpha}\right|_{h}^{2} \leq k_{\alpha} \leq C_{13}\left|\rho_{\alpha}\right|_{h}^{2}$ on $\Delta\left(R_{1}\right)$.

According to Lemma 2.8 below, there exists a constant $\epsilon_{1}>0$ depending only on $R, R_{1}, r, C_{10}$ such that we have $\left|\left[f_{h}^{\dagger}, \pi_{\alpha}\right]\right|_{h} \geq \epsilon_{1} d\left|\rho_{\alpha}\right|_{h}$ on $\Delta\left(R_{1}\right)$. Hence, we have a constant $\epsilon_{2}>0$ depending only on $R, R_{1}, r, C_{10}$ such that the following holds on $\Delta\left(R_{1}\right)$ :

$$
-\partial_{z} \partial_{\bar{z}} k_{\alpha} \leq-\epsilon_{2} d^{2} k_{\alpha}
$$

For any positive number $\epsilon_{3}>0$, we have the following on $\Delta\left(R_{1}\right)$ :

$$
-\partial_{z} \partial_{\bar{z}} \exp \left(\epsilon_{3} d|z|^{2}\right)=-\left(d \epsilon_{3}+d^{2}|z|^{2} \epsilon_{3}^{2}\right) \exp \left(\epsilon_{3} d|z|^{2}\right) \geq-\left(d \epsilon_{3}+d^{2} \epsilon_{3}^{2} R_{1}^{2}\right) \exp \left(\epsilon_{3} d|z|^{2}\right)
$$

Take $\epsilon_{3}>0$ such that $\epsilon_{3} \leq\left(\epsilon_{2} R_{1}^{-1}\right) / 2$ and $\epsilon_{3} \leq d \epsilon_{2} / 2$. Then, we have

$$
-\partial_{z} \partial_{\bar{z}} \exp \left(\epsilon_{3} d|z|^{2}\right) \geq-\epsilon_{2} d^{2} \exp \left(\epsilon_{3} d|z|^{2}\right) .
$$

We take $C_{14}>0$ depending only on $R, R_{1}, r, C_{10}$ such that $k_{\alpha}(P)<C_{14}$ for $|z(P)|=R_{1}$. We set

$$
\mathcal{Z}:=\left\{P \in \Delta\left(R_{1}\right) \mid k_{\alpha}(P)>C_{14} \exp \left(\epsilon_{3} d|z|^{2}-\epsilon_{3} d R_{1}^{2}\right)(P)\right\} .
$$

Suppose that $\mathcal{Z}$ is non-empty. By the choice of $C_{14}, \mathcal{Z}$ is relatively compact in $\Delta\left(R_{1}\right)$. So, we have $k_{\alpha}=$ $C_{14} \exp \left(\epsilon_{3} d|z|^{2}-\epsilon_{3} d R_{1}^{2}\right)$ on the boundary of $\mathcal{Z}$. We also have the following inequality on $\mathcal{Z}$ :

$$
-\partial_{z} \partial_{\bar{z}}\left(k_{\alpha}-C_{14} \exp \left(\epsilon_{3} d|z|^{2}-\epsilon_{3} d R_{1}^{2}\right)\right) \leq-\epsilon_{2} d^{2}\left(k_{\alpha}-C_{14} \exp \left(\epsilon_{3} d|z|^{2}-\epsilon_{3} d R_{1}^{2}\right)\right) \leq 0
$$

We obtain $k_{\alpha} \leq C_{14} \exp \left(\epsilon_{3} d|z|^{2}-\epsilon_{3} d R_{1}^{2}\right)$ on $\mathcal{Z}$, which contradicts with the construction of $\mathcal{Z}$. Hence, we obtain that $\mathcal{Z}=\emptyset$, i.e., $k_{\alpha} \leq C_{14} \exp \left(\epsilon_{3} d|z|^{2}-\epsilon_{3} d R_{1}^{2}\right)=C_{14} \exp \left(-\epsilon_{3}\left(R_{1}^{2}-|z|^{2}\right) d\right)$ holds on $\Delta\left(R_{1}\right)$. We obtain the following on $\Delta\left(R_{2}\right)$ :

$$
k_{\alpha} \leq C_{14} \exp \left(-\epsilon_{3}\left(R_{1}^{2}-R_{2}^{2}\right) d\right)
$$

Then, by setting $C_{11}:=C_{14}>0$ and $\epsilon_{0}:=\epsilon_{3}\left(R_{1}^{2}-R_{2}^{2}\right)>0$, we obtain the claim of Proposition 2.3.

For any endomorphism $g$ of $E$, let $g_{h}^{\dagger}$ denote the adjoint of $g$ with respect to $h$. We also denote it by $g^{\dagger}$ if there is no risk of confusion.

Corollary 2.5 There exist positive constants $C_{20}$ and $\epsilon_{20}$, depending only on $R, R_{1}, R_{2}, r$ and $C_{10}$ such that $\left|\left[f,\left(\pi_{\alpha}\right)_{h}^{\dagger}\right]\right|_{h}=\left|\left[f_{h}^{\dagger}, \pi_{\alpha}\right]\right|_{h} \leq C_{20} \exp \left(-\epsilon_{20} d\right)$ on $\Delta\left(R_{2}\right)$.

Proof We have $\left|\rho_{\alpha}\right|_{h}=\left|\pi_{\alpha}-\pi_{\alpha}^{\prime}\right|_{h} \leq C_{11} \exp \left(-\epsilon_{0} d\right)$. We also have $\left|\left(\rho_{\alpha}\right)_{h}^{\dagger}\right|_{h}=\left|\left(\pi_{\alpha}\right)_{h}^{\dagger}-\pi_{\alpha}^{\prime}\right|_{h} \leq C_{11} \exp \left(-\epsilon_{0} d\right)$. We obtain

$$
\left|\left[f, \pi_{\alpha}^{\dagger}\right]\right|_{h}=\left|\left[f, \pi_{\alpha}^{\dagger}-\pi_{\alpha}\right]\right|_{h} \leq\left|\left[f, \rho_{\alpha}^{\dagger}\right]\right|_{h}+\left|\left[f, \rho_{\alpha}\right]\right|_{h} \leq 2\left(C_{1} M+C_{2}\right) C_{11} \exp \left(-\epsilon_{0} d\right) .
$$

Then, we obtain the desired estimate.

Corollary 2.6 There exist positive constants $C_{21}$ and $\epsilon_{21}$ depending only on $R, R_{1}, R_{2}, r$ and $C_{10}$, such that the following holds:

- Take any $\alpha, \beta \in S$ with $\alpha \neq \beta$. Let $s_{\alpha}$ and $s_{\beta}$ be local sections of $E_{\alpha}$ and $E_{\beta}$. Then, we have

$$
\left|h\left(s_{\alpha}, s_{\beta}\right)\right| \leq C_{21} \exp \left(-\epsilon_{21} d\right) \cdot\left|s_{\alpha}\right|_{h} \cdot\left|s_{\beta}\right|_{h}
$$

Proof We have $h\left(s_{\alpha}, s_{\beta}\right)=h\left(\pi_{\alpha} s_{\alpha}, s_{\beta}\right)=h\left(s_{\alpha}, \pi_{\alpha}^{\dagger} s_{\beta}\right)=h\left(s_{\alpha},\left(\pi_{\alpha}^{\dagger}-\pi_{\alpha}\right) s_{\beta}\right)$. We have $\pi_{\alpha}^{\dagger}-\pi_{\alpha}=\rho_{\alpha}^{\dagger}-\rho_{\alpha}$, where $\rho_{\alpha}^{\dagger}$ denote the adjoint of $\rho_{\alpha}$ with respect to $h$. Then, we obtain (3) from Proposition 2.3. 


\subsubsection{Appendix: Preliminary from linear algebra}

Fix positive constants $G_{i}(i=1,2)$. Fix a positive integer $r>0$. Let $U$ be any $r$-dimensional $\mathbb{C}$-vector space with a Hermitian metric $h$. Let $f$ be an endomorphism on $U$. Suppose that we have a finite subset $S \subset \mathbb{C}$ and a decomposition $(U, f)=\bigoplus_{\alpha \in S}\left(U_{\alpha}, f_{\alpha}\right)$, which is not necessarily orthogonal. We impose the following conditions.

- We set $d:=\min \{|\alpha-\beta| \mid \alpha, \beta \in S, \alpha \neq \beta\}$. Then, we have $d \geq 1$ and $|f|_{h} \leq G_{1} d+G_{2}$.

- The eigenvalues $\gamma$ of $f_{\alpha}$ satisfy $|\gamma-\alpha| \leq d / 100$.

Let $\pi_{\alpha}$ be the projection with respect to the decomposition $U=\bigoplus U_{\alpha}$. Let $\pi_{\alpha}^{\prime}$ denote the orthogonal projection of $U$ onto $U_{\alpha}$. We set $\rho_{\alpha}:=\pi_{\alpha}-\pi_{\alpha}^{\prime}$. To clarify the argument, we recall the following lemma from [20] which was used in $[13,16]$.

Lemma 2.7 There exists a positive constant $B_{1}$ depending only on $r$ and $G_{i}(i=1,2)$ such that

$$
\left|\pi_{\alpha}\right|_{h} \leq B_{1}, \quad\left|\rho_{\alpha}\right|_{h} \leq B_{1} .
$$

Proof Because $\rho_{\alpha}$ and $\pi_{\alpha}^{\prime}$ are orthogonal, we have $\left|\rho_{\alpha}\right|_{h} \leq\left|\pi_{\alpha}\right|_{h}$. Hence, it is enough to obtain the estimate for $\pi_{\alpha}$. Let $\operatorname{id}_{U}$ denote the identity on $U$. We have

$$
\pi_{\alpha}=\frac{1}{2 \pi \sqrt{-1}} \int_{\gamma_{\alpha}}\left(\zeta \operatorname{id}_{U}-f\right)^{-1} d \zeta
$$

Here, $\gamma_{\alpha}$ denotes the loop $\gamma_{\alpha}(\theta)=\alpha+d e^{\sqrt{-1} \theta} / 10(0 \leq \theta \leq 2 \pi)$. There exist positive constants $B_{i}(i=2,3)$ depending only on $r$ and $G_{i}(i=1,2)$ such that

$$
\left|\left(\gamma_{\alpha}(\theta) \operatorname{id}_{U}-f\right)^{-1}\right|_{h} \leq d^{-1} B_{2}\left(\left(G_{1}+d^{-1} G_{2}\right)^{r}+1\right) \leq d^{-1} B_{3} .
$$

Thus, we obtain the claim of the lemma.

Let $f_{h}^{\dagger}$ denote the adjoint of $f$ with respect to $h$. To clarify our argument, we recall the following lemma from $[20]$ which was used in $[13,16]$.

Lemma 2.8 We have $\delta>0$ depending only on $r$ and $G_{i}(i=1,2)$ such that $\left|\left[f_{h}^{\dagger}, \pi_{\alpha}\right]\right|_{h} \geq \delta \cdot d \cdot\left|\rho_{\alpha}\right|_{h}$.

Proof We take a numbering of the elements of $S$, i.e., $S=\left\{\alpha_{1}, \ldots, \alpha_{m}\right\}$. We impose $\alpha_{1}=\alpha$. We set $F_{j}(U):=\bigoplus_{i \leq j} U_{\alpha_{i}}$ and $F_{<j}(U):=\bigoplus_{i<j} U_{\alpha_{i}}$. Let $U_{j}^{\prime}$ be the orthogonal complement of $F_{<j}(U)$ in $F_{j}(U)$. We have the orthogonal decomposition $F_{j}(U)=\bigoplus_{i \leq j} U_{i}^{\prime}$. Because $f\left(F_{j}\right) \subset F_{j}$, we have the decomposition $f=\sum_{i \leq j} f_{i j}$, where $f_{i j}: U_{j}^{\prime} \longrightarrow U_{i}^{\prime}$. As the adjoint, we have $f_{h}^{\dagger}=\sum_{i \leq j}\left(f_{i j}\right)_{h}^{\dagger}$, where $\left(f_{i j}\right)_{h}^{\dagger}: U_{i}^{\prime} \longrightarrow U_{j}^{\prime}$. We set $f_{i j}^{\dagger}:=\left(f_{j i}\right)_{h}^{\dagger}$. Then, we have $f_{h}^{\dagger}=\sum_{i \geq j} f_{i j}^{\dagger}$, where $f_{i j}^{\dagger}: U_{j}^{\prime} \longrightarrow U_{i}^{\prime}$.

For each $i$, we take an orthonormal base $e_{1}^{(i)}, \ldots, e_{r_{i}}^{(i)}$ of $U_{i}^{\prime}$ for which $f_{i i}^{\dagger}$ is represented by a lower triangular matrix $A_{i}$. Let $\Gamma_{i} \in \operatorname{End}\left(U_{i}^{\prime}\right)$ be determined by $\Gamma_{i}\left(e_{k}^{(i)}\right)=\alpha_{k}^{(i)} e_{k}^{(i)}$, where $\alpha_{k}^{(i)}$ denote the $(k, k)$-entry of $A_{i}$. Then, $f_{i i}^{\dagger}-\Gamma_{i}$ is nilpotent. We put $\Gamma=\sum \Gamma_{i} \in \operatorname{End}(U)$. Then, $f_{h}^{\dagger}-\Gamma$ is nilpotent, and we have $\left|f_{h}^{\dagger}-\Gamma\right|_{h} \leq G_{1} d+G_{2}$.

Let $\Pi$ denote the orthogonal projection of $\operatorname{End}(E) \stackrel{=}{=} \operatorname{Hom}\left(U_{j}^{\prime}, U_{i}^{\prime}\right)$ onto $\bigoplus_{j>i} \operatorname{Hom}\left(U_{j}^{\prime}, U_{i}^{\prime}\right)$. Let $F_{1}$ be the endomorphism on $\bigoplus_{j>i} \operatorname{Hom}\left(U_{j}^{\prime}, U_{i}^{\prime}\right)$ given by $F_{1}(B):=\Pi\left(\left[f_{h}^{\dagger}, B\right]\right)$. Let $F_{2}$ be the endomorphism on $\bigoplus_{j>i} \operatorname{Hom}\left(U_{j}^{\prime}, U_{i}^{\prime}\right)$ given by $F_{2}(B):=[\Gamma, B]=\Pi([\Gamma, B])$.

Let us observe that $F_{1}-F_{2}$ is nilpotent. We have the nilpotent endomorphism $\widetilde{F}$ on $\operatorname{End}(E)$ given by $\widetilde{F}(B)=\left[f_{h}^{\dagger}-\Gamma, B\right]$, which preserves $\bigoplus_{j \leq i} \operatorname{Hom}\left(U_{j}^{\prime}, U_{i}^{\prime}\right)$. Then, $F_{1}-F_{2}$ is equal to the endomorphism on $\bigoplus_{j>i} \operatorname{Hom}\left(U_{j}^{\prime}, U_{i}^{\prime}\right) \simeq \operatorname{End}(E) / \bigoplus_{j \leq i} \operatorname{Hom}\left(U_{j}^{\prime}, U_{i}^{\prime}\right)$ induced by $\widetilde{F}$, and hence $F_{1}-F_{2}$ is nilpotent.

There exists a constant $B_{11}>0$ depending only on $r$ such that $\left|F_{1}-F_{2}\right|_{h} \leq B_{11}\left(G_{1} d+G_{2}\right)$. Moreover, $F_{2}$ is invertible, and there exists a constant $B_{12}>0$ depending only on $r$ such that $\left|F_{2}^{-1}\right|_{h} \leq B_{12} d^{-1}$. Hence, we obtain that $\left|F_{1}^{-1}\right|_{h} \leq B_{13} d^{-1}\left(1+\left(G_{1}+G_{2} / d\right)^{r}\right)$ for a constant $B_{13}>0$ depending only on $r$. 
Note that we have $\Pi\left(\left[f_{h}^{\dagger}, \pi_{\alpha}^{\prime}\right]\right)=0$. Hence, we have the following:

$$
\left|\left[f_{h}^{\dagger}, \pi_{\alpha}\right]\right|_{h} \geq\left|\Pi\left(\left[f_{h}^{\dagger}, \pi_{\alpha}\right]\right)\right|_{h}=\left|\Pi\left(\left[f_{h}^{\dagger}, \rho_{\alpha}\right]\right)\right|_{h}=\left|F_{1}\left(\rho_{\alpha}\right)\right|_{h} \geq B_{13}^{-1} d\left(1+\left(G_{1}+G_{2} d^{-1}\right)^{r}\right)^{-1}\left|\rho_{\alpha}\right|_{h}
$$

Thus, we obtain the claim of the lemma.

\section{$2.2 \quad$ Asymptotic decoupling of harmonic bundles on discs}

We continue to use the setting in $\S 2.1$. We further impose the following condition:

- $\operatorname{rank} E_{\alpha}=1$ for each $\alpha \in S$.

In other words, we assume to have holomorphic functions $g_{\alpha}(\alpha \in S)$ on $\Delta(R)$ such that $\theta=\bigoplus g_{\alpha} \operatorname{id}_{E_{\alpha}} d z$. (Recall the generically regular semisimple condition in §1.1.1.) By the condition, we have $\left|g_{\alpha}(P)-\alpha\right| \leq d / 100$. In this setting, we explain that Simpson's main estimate implies the asymptotic decoupling of the Hitchin equation.

Let $g_{\mathbb{C}}$ denote the Euclidean metric $d z d \bar{z}$ of $\mathbb{C}$. For any section $s$ of $\operatorname{End}(E) \otimes \Omega^{p, q}$ on $\Delta\left(R^{\prime}\right)\left(R^{\prime}>0\right)$, let $|s|_{h, g_{\mathbb{C}}}$ denote the function on $\Delta\left(R^{\prime}\right)$ by taking the norm of $s$ at each $P \in \Delta\left(R^{\prime}\right)$ with respect to $h$ and $g_{\mathbb{C}}$.

\subsubsection{Decay of the curvatures}

Let $R(h)$ denote the curvature of the Chern connection of $\left(E, \bar{\partial}_{E}, h\right)$. We obtain the following "asymptotic decoupling" of the Hitchin equation $R(h)+\left[\theta, \theta_{h}^{\dagger}\right]=0$.

Theorem 2.9 There exist positive constants $C_{30}$ and $\epsilon_{30}$, depending only on $R, R_{1}, R_{2}, r$ and $C_{10}$ such that $|R(h)|_{h, g_{\mathrm{C}}}=\left|\left[\theta, \theta_{h}^{\dagger}\right]\right|_{h, g_{\mathrm{C}}} \leq C_{30} \exp \left(-\epsilon_{30} d\right)$ on $\Delta\left(R_{2}\right)$.

Proof It is enough to obtain the estimate for $\left[\theta, \theta_{h}^{\dagger}\right]$. We have the decomposition $f_{h}^{\dagger}=\sum_{\alpha, \beta} \pi_{\alpha} \circ f_{h}^{\dagger} \circ \pi_{\beta}$. By Corollary 2.5, if $\alpha \neq \beta$, we have $C_{31}>0$, depending only on $R, R_{1}, R_{2}, r$ and $C_{10}$, such that

$$
\left|\pi_{\alpha} \circ f_{h}^{\dagger} \circ \pi_{\beta}\right|_{h}=\left|\left[\pi_{\alpha}, f_{h}^{\dagger}\right] \circ \pi_{\beta}\right|_{h} \leq C_{31} \exp \left(-\epsilon_{20} d\right) .
$$

For $\alpha \neq \beta$, we have $\left[f_{\alpha}, \pi_{\beta} \circ f_{h}^{\dagger} \circ \pi_{\beta}\right]=0$. Because $\operatorname{rank} E_{\alpha}=1$, we also have $\left[f_{\alpha}, \pi_{\alpha} \circ f_{h}^{\dagger} \circ \pi_{\alpha}\right]=0$. Then, we obtain the estimate for $\left[\theta, \theta_{h}^{\dagger}\right]$.

\subsubsection{The connections and the projections}

Let $\partial_{E}$ denote the $(1,0)$-part of the Chern connection associated to $h$ and $\bar{\partial}_{E}$. According to Proposition 2.3, the decomposition $E=\bigoplus E_{\alpha}$ is almost orthogonal. Let us see that such an almost orthogonality holds at the level of the first derivative in the sense that $\partial_{E} \pi_{\alpha}$ is very small.

Proposition 2.10 Take $0<R_{3}<R_{2}$. There exist positive constants $\epsilon_{40}$ and $C_{40}$ depending only on $R, R_{1}$, $R_{2}, R_{3}, r$ and $C_{10}$ such that $\left|\partial_{E} \pi_{\alpha}\right|_{h, g_{\mathrm{C}}}=\left|\bar{\partial}_{E} \pi_{\alpha}^{\dagger}\right|_{h, g_{\mathbb{C}}} \leq C_{40} \exp \left(-\epsilon_{40} d\right)$ on $\Delta\left(R_{3}\right)$.

Proof It is enough to obtain the estimate for $\partial_{E} \pi_{\alpha}$. In the following, the constants may depend only on $R$, $R_{1}, R_{2}, r$ and $C_{10}$. We have $C_{41}>0$ such that the following holds on $\Delta\left(R_{2}\right)$ :

$$
\left|\bar{\partial}_{E} \partial_{E} \pi_{\alpha}\right|_{h, g_{\mathrm{C}}}=\left|\left[R(h), \pi_{\alpha}\right]\right|_{h, g_{\mathrm{C}}} \leq C_{41} \exp \left(-\epsilon_{30} d\right) .
$$

Because $\partial_{E} \pi_{\alpha}^{\dagger}=0$, we have the following on $\Delta\left(R_{2}\right)$ :

$$
\left|\bar{\partial}_{E} \partial_{E}\left(\pi_{\alpha}-\pi_{\alpha}^{\dagger}\right)\right|_{h, g_{\mathbb{C}}} \leq C_{41} \exp \left(-\epsilon_{30} d\right)
$$

We already have $\left|\pi_{\alpha}-\pi_{\alpha}^{\dagger}\right|_{h} \leq C_{42} \exp \left(-\epsilon_{0} d\right)$ for a constant $C_{42}>0$. We may assume $\epsilon_{30}<\epsilon_{0}$. Hence, we have a constant $C_{43}>0$ such that $\left\|\pi_{\alpha}-\pi_{\alpha}^{\dagger}\right\|_{h, g_{\mathrm{C}}, L_{2}^{p}} \leq C_{43} \exp \left(-\epsilon_{30} d\right)$ for a large $p>1$. Here, $\|\cdot\|_{h, g_{\mathrm{C}}, L_{2}^{p}}$ denote the $L_{2}^{p}$-norm with respect to $h$ and $g_{\mathbb{C}}$ on $\Delta\left(R_{2}\right)$. Then, we obtain the claim of the lemma.

Recall that $\pi_{\alpha}^{\prime}$ denote the orthogonal projection of $E$ onto $E_{\alpha}$. Let us see that it is almost holomorphic. 
Proposition 2.11 We have a positive constant $C_{50}$, depending only on $R, R_{1}, R_{2}, R_{3}, r$ and $C_{10}$ such that the following holds on $\Delta\left(R_{3}\right)$ :

$$
\left|\bar{\partial}_{E} \pi_{\alpha}^{\prime}\right|_{h, g_{\mathrm{C}}}=\left|\partial_{E} \pi_{\alpha}^{\prime}\right|_{h, g_{\mathrm{C}}} \leq C_{50} \exp \left(-\epsilon_{40} d\right) .
$$

Proof It is enough to prove the estimate for $\bar{\partial}_{E} \pi_{\alpha}^{\prime}$. Let $E=E_{\alpha} \oplus E_{\alpha}^{\perp}$ denote the orthogonal decomposition. We may naturally regard $\rho_{\alpha}:=\pi_{\alpha}-\pi_{\alpha}^{\prime}$ as a morphism $E_{\alpha}^{\perp} \longrightarrow E_{\alpha}$. We may also regard $\rho_{\alpha}^{\dagger}:=\pi_{\alpha}^{\dagger}-\pi_{\alpha}^{\prime}$ as a morphism $E_{\alpha} \longrightarrow E_{\alpha}^{\perp}$.

We have the induced holomorphic structure on $E_{\alpha}^{\perp} \simeq E / E_{\alpha}$. Let $\bar{\partial}_{E}^{(0)}$ be the holomorphic structure on $E_{\alpha} \oplus E_{\alpha}^{\perp}$ obtained as the direct sum. We have $\bar{\partial}_{E}=\bar{\partial}_{E}^{(0)}+\kappa$, where $\kappa$ is a section of $\operatorname{Hom}\left(E_{\alpha}^{\perp}, E_{\alpha}\right) \otimes \Omega^{0,1}$. We have

$$
\bar{\partial}_{E}\left(\pi_{\alpha}-\pi_{\alpha}^{\dagger}\right)=\bar{\partial}_{E} \rho_{\alpha}-\bar{\partial}_{E} \rho_{\alpha}^{\dagger}=\bar{\partial}_{E}^{(0)} \rho_{\alpha}-\bar{\partial}_{E}^{(0)} \rho_{\alpha}^{\dagger}-\kappa \circ \rho_{\alpha}^{\dagger}+\rho_{\alpha}^{\dagger} \circ \kappa
$$

Note that $\bar{\partial}_{E}^{(0)}\left(\rho_{\alpha}\right), \bar{\partial}_{E}^{(0)}\left(\rho_{\alpha}^{\dagger}\right), \kappa \circ \rho_{\alpha}^{\dagger}$ and $\rho_{\alpha}^{\dagger} \circ \kappa$ are sections of $\operatorname{Hom}\left(E_{\alpha}^{\perp}, E_{\alpha}\right) \otimes \Omega^{0,1}, \operatorname{Hom}\left(E_{\alpha}, E_{\alpha}^{\perp}\right) \otimes \Omega^{0,1}$, $\operatorname{Hom}\left(E_{\alpha}, E_{\alpha}\right) \otimes \Omega^{0,1}$ and $\operatorname{Hom}\left(E_{\alpha}^{\perp}, E_{\alpha}^{\perp}\right) \otimes \Omega^{0,1}$, respectively. Note that the bundles are orthogonal with respect to $h$ and $g_{\mathbb{C}}$. In general, for any orthogonal decomposition of bundles $\mathcal{V}=\bigoplus \mathcal{V}_{i}$ and for any section $s=\sum s_{i}$ of $\mathcal{V}$, the norms of $s_{i}$ are smaller than the norm of $s$. Hence, we obtain the following from (4):

$$
\left|\bar{\partial}_{E}^{(0)} \rho_{\alpha}\right|_{h, g_{\mathrm{C}}} \leq\left|\bar{\partial}_{E}\left(\pi_{\alpha}-\pi_{\alpha}^{\dagger}\right)\right|_{h, g_{\mathrm{C}}}=\left|\bar{\partial}_{E}\left(\pi_{\alpha}^{\dagger}\right)\right|_{h, g_{\mathrm{C}}}
$$

We also have $\bar{\partial}_{E} \pi_{\alpha}^{\prime}=-\bar{\partial}_{E} \rho_{\alpha}=-\bar{\partial}_{E}^{(0)} \rho_{\alpha}$. Hence, we obtain $\left|\bar{\partial}_{E} \pi_{\alpha}^{\prime}\right|_{h, g_{\mathrm{C}}} \leq\left|\bar{\partial}_{E}\left(\pi_{\alpha}^{\dagger}\right)\right|_{h, g_{\mathrm{C}}}$ from (5). Then, the claim of Proposition 2.11 follows from the estimate in Proposition 2.10.

\subsubsection{The decay of the curvatures on the line bundles}

Let $\bar{\partial}_{\alpha}$ denote the holomorphic structure of $E_{\alpha}$. Let $h_{\alpha}$ be the restriction of $h$ to $E_{\alpha}$. Let $\partial_{\alpha}$ denote the $(1,0)$-part of the Chern connection of $\left(E_{\alpha}, \bar{\partial}_{\alpha}, h_{\alpha}\right)$. Let $R\left(h_{\alpha}\right)$ denote the curvature of the connection $\bar{\partial}_{\alpha}+\partial_{\alpha}$. We have $\partial_{\alpha} s=\pi_{\alpha}^{\prime} \circ \partial_{E} s$ and $\bar{\partial}_{\alpha} s=\bar{\partial}_{E} s$ for any section $s$ of $E_{\alpha}$.

Proposition 2.12 We have a positive constant $C_{60}$ depending only on $R, R_{1}, R_{2}, R_{3}, r$ and $C_{10}$ such that $\left|R\left(h_{\alpha}\right)\right|_{h_{\alpha}, g_{\mathrm{C}}} \leq C_{60} \exp \left(-\epsilon_{40} d\right)$ on $\Delta\left(R_{3}\right)$.

Proof In the following, the constants may depend only on $R, R_{1}, R_{2}, R_{3}, r$ and $C_{10}$. Let $s$ be any section of $E_{\alpha}$. We have

$$
\bar{\partial}_{E} \circ \pi_{\alpha}^{\prime}\left(\partial_{E} s\right)=\bar{\partial}_{E} \circ \pi_{\alpha}^{\prime} \circ \partial_{E}\left(\pi_{\alpha} s\right)=\bar{\partial}_{E} \circ \pi_{\alpha}\left(\partial_{E} s\right)+\bar{\partial}_{E} \circ \pi_{\alpha}^{\prime} \circ\left(\partial_{E} \pi_{\alpha}\right)(s)=\pi_{\alpha}\left(\bar{\partial}_{E} \partial_{E} s\right)+\bar{\partial}_{E}\left(\pi_{\alpha}^{\prime}\left(\partial_{E} \pi_{\alpha}\right) s\right) .
$$

We also have

$$
\pi_{\alpha}^{\prime} \circ \partial_{E} \circ \bar{\partial}_{E} s=\pi_{\alpha}^{\prime} \circ \partial_{E}\left(\pi_{\alpha} \bar{\partial}_{E} s\right)=\pi_{\alpha} \partial_{E} \bar{\partial}_{E} s+\pi_{\alpha}^{\prime} \partial_{E}\left(\pi_{\alpha}\right) \bar{\partial}_{E} s .
$$

Hence, it is enough to obtain an estimate of $\bar{\partial}_{E}\left(\pi_{\alpha}^{\prime} \circ \partial_{E} \pi_{\alpha}\right)=\bar{\partial}_{E}\left(\pi_{\alpha}^{\prime}\right) \circ \partial_{E}\left(\pi_{\alpha}\right)+\pi_{\alpha}^{\prime} \circ \bar{\partial}_{E} \partial_{E} \pi_{\alpha}$. By Proposition 2.10 and Proposition 2.11, we have $C_{61}>0$ such that $\left|\bar{\partial}_{E}\left(\pi_{\alpha}^{\prime}\right) \circ \partial_{E}\left(\pi_{\alpha}\right)\right|_{h, g_{\mathrm{C}}} \leq C_{61} \exp \left(-\epsilon_{40} d\right)$. We also have $\left|\pi_{\alpha}^{\prime} \circ \bar{\partial}_{E} \partial_{E} \pi_{\alpha}\right|_{h, g_{\mathrm{C}}}=\left|\pi_{\alpha}^{\prime} \circ\left[R(h), \pi_{\alpha}\right]\right|_{h, g_{\mathrm{C}}} \leq C_{62} \exp \left(-\epsilon_{40} d\right)$ for a constant $C_{62}>0$. Then, the claim of the lemma follows.

\subsubsection{Approximation of the flat connections}

We consider the flat connection $\mathbb{D}^{1}:=\bar{\partial}_{E}+\partial_{E}+\theta+\theta^{\dagger}$ on $E$. We also have the connection $\mathbb{D}_{0}^{1}$ on $E=\bigoplus_{\alpha \in S} E_{\alpha}$ given by

$$
\mathbb{D}_{0}^{1}=\bigoplus_{\alpha \in S}\left(\left(\bar{\partial}_{\alpha}+\partial_{\alpha}\right)+\left(g_{\alpha} d z+\overline{g_{\alpha}} d \bar{z}\right) \operatorname{id}_{E_{\alpha}}\right) .
$$

Here, $g_{\alpha}$ are holomorphic functions such that $\theta=\bigoplus g_{\alpha} \operatorname{id}_{E_{\alpha}} d z$, as in the beginning of $\S 2.2$. 
Corollary 2.13 There exists a positive constant $C_{70}$, depending only on $R, R_{1}, R_{2}, R_{3}, r$ and $C_{10}$ such that $\left|\mathbb{D}^{1}-\mathbb{D}_{0}^{1}\right|_{h, g_{\mathbb{C}}} \leq C_{70} \exp \left(-\epsilon_{40} d\right)$ on $\Delta\left(R_{3}\right)$.

Proof It is enough to obtain an estimate of $\partial_{E}-\bigoplus_{\alpha \in S} \partial_{\alpha}$. We have a constant $C_{71}>0$, depending only on $R, R_{1}, R_{2}, R_{3}, r$ and $C_{10}$ such that $\left|\pi_{\alpha}^{\prime} \circ\left(\partial_{E} \pi_{\alpha}\right)\right|_{h, g_{\mathbb{C}}} \leq C_{71} \exp \left(-\epsilon_{40} d\right)$. Hence, for any section $s$ of $E_{\alpha}$, we have

$$
\left|\pi_{\alpha} \circ \partial_{E} s-\partial_{\alpha} s\right|_{h, g_{\mathbb{C}}}=\left|\pi_{\alpha}^{\prime} \circ \pi_{\alpha} \circ \partial_{E} s-\pi_{\alpha}^{\prime} \partial_{E}\left(\pi_{\alpha} s\right)\right|_{h, g_{\mathbb{C}}}=\left|\pi_{\alpha}^{\prime} \circ \partial_{E} \pi_{\alpha}(s)\right|_{h, g_{\mathbb{C}}} \leq C_{71} \exp \left(-\epsilon_{40} d\right)|s|_{h} .
$$

Then, the claim of the lemma follows.

\subsubsection{Higher derivatives}

We take a numbering $\left\{\alpha_{1}, \ldots, \alpha_{r}\right\}$ on $S$. For each $i$, we can take a holomorphic frame $u_{i}$ of $E_{\alpha_{i}}$ such that $\left|u_{i \mid 0}\right|_{h}=1$ and $\left|\partial_{\alpha_{i}} u_{i}\right|_{h, g_{\mathbb{C}}} \leq C_{80} \exp \left(-\epsilon_{80} d\right)$ on $\Delta\left(R_{3}\right)$ for some positive constants $C_{80}$ and $\epsilon_{80}$, depending only on $R, R_{1}, R_{2}, R_{3}, r$ and $C_{10}$. Let us sketch how to obtain such sections in an elementary way. For a real coordinate $z=x+\sqrt{-1} y$, we can take a section $s_{i}$ of $E_{\alpha_{i}}$ on $\Delta\left(R_{2}\right)$ such that $\left(\bar{\partial}_{\alpha_{i}}+\partial_{\alpha_{i}}\right) s_{i}=s_{i} \cdot \nu_{i} d x=$ $s_{i} \cdot \nu_{i}(d z+d \bar{z}) / 2$ such that $\nu_{i}(x, 0)=0$. The curvature form is given by $\partial_{y} \nu_{i} d y d x$. By Proposition 2.12 , we have $\left|\nu_{i}\right| \leq C_{80}^{\prime} \exp \left(-\epsilon_{80}^{\prime} d\right)$. Take $R_{2}^{\prime}$ such that $R_{3}<R_{2}^{\prime}<R_{2}$. We can take a function $\rho_{i}$ on $\Delta\left(R_{2}^{\prime}\right)$ satisfying $\partial_{\bar{z}} \rho_{i}=\nu_{i} / 2,\left|\rho_{i}\right| \leq C_{80}^{\prime \prime} \exp \left(-\epsilon_{80}^{\prime \prime} d\right)$ and $\left\|\partial_{z} \rho_{i}\right\|_{L^{p}\left(\Delta\left(R_{2}^{\prime}\right)\right)} \leq C_{80, p}^{\prime \prime} \exp \left(-\epsilon_{80}^{\prime \prime}\right)$ for any $p \geq 1$. We set $v_{i}:=s_{i} e^{-\rho_{i}}$ on $\Delta\left(R_{2}^{\prime}\right)$. Then, we have $\bar{\partial}_{\alpha_{i}} v_{i}=0$ and $\partial_{\alpha_{i}} v_{i}=v_{i} \cdot \kappa_{i} d z$, where $\left\|\kappa_{i}\right\|_{L^{p}\left(\Delta\left(R_{2}^{\prime}\right)\right)} \leq C_{80, p}^{(3)} \exp \left(-\epsilon_{80}^{(3)} d\right)$. Because $\partial_{\bar{z}} \kappa_{i} d \bar{z} d z$ is the curvature of $E_{\alpha}$, we have $\left|\partial_{\bar{z}} \kappa_{i}\right| \leq C_{80}^{(4)} \exp \left(-\epsilon_{80}^{(4)} d\right)$. Hence, we obtain $\left|\kappa_{i}\right| \leq C_{80}^{(5)} \exp \left(-\epsilon_{80}^{(5)} d\right)$ on $\Delta\left(R_{3}\right)$. By adjusting the norm of $v_{i}$ at the origin, we obtain the desired section $u_{i}$. Note that we have $\left.|\log | u_{i}\right|_{h} \mid \leq C_{81} \exp \left(-\epsilon_{81} d\right)$ for some positive constants $C_{81}$ and $\epsilon_{81}$ depending only on $R, R_{1}, R_{2}, R_{3}, r$ and $C_{10}$.

We obtain a frame $\boldsymbol{u}=\left(u_{1}, \ldots, u_{r}\right)$ of $E$ on $\Delta\left(R_{3}\right)$. Let $H$ be the $r$-square Hermitian matrix valued function given by $H_{i j}=h\left(u_{i}, u_{j}\right)$. Let $\Theta$ be the holomorphic $r$-square matrix valued function such that $\Theta_{i i}=g_{\alpha_{i}}$ and $\Theta_{i j}=0(i \neq j)$. We have $\theta \boldsymbol{u}=\boldsymbol{u} \cdot \Theta d z$. Let $\Theta^{\dagger}$ be the $r$-square matrix valued function given by $\Theta^{\dagger}=\bar{H}^{-1} \epsilon_{\Theta H}$. We have $\theta_{h}^{\dagger} \boldsymbol{u}=\boldsymbol{u} \Theta^{\dagger} d \bar{z}$.

Because $H_{i i}=\left|u_{i}\right|_{h}^{2}$, we have $\left|\log H_{i i}\right| \leq 2 C_{81} \exp \left(-\epsilon_{81} d\right)$, as remarked above. We also have positive constants $C_{82}$ and $\epsilon_{82}$ depending only on $R, R_{1}, R_{2}, R_{3}, r$ and $C_{10}$ such that the following holds:

$$
\begin{gathered}
\left|H_{i j}\right| \leq C_{82} \exp \left(-\epsilon_{82} d\right), \quad(i \neq j) \\
\left|\partial_{z} H_{i j}\right|=\left|\partial_{\bar{z}} H_{i j}\right| \leq C_{82} \exp \left(-\epsilon_{82} d\right) \\
\left|\partial_{\bar{z}} \partial_{z} H_{i j}\right| \leq C_{82} \exp \left(-\epsilon_{82} d\right)
\end{gathered}
$$

Indeed, (6) follows from Corollary 2.6. We have $\partial h\left(u_{i}, u_{j}\right)=h\left(\left(\partial_{E} \pi_{\alpha_{i}}\right) u_{i}, u_{j}\right)+h\left(\pi_{\alpha_{i}}\left(\partial_{E} u_{i}\right), u_{j}\right)$. As in the proof of Corollary 2.13, we have $\left|\pi_{\alpha_{i}} \partial_{E} u_{i}-\partial_{\alpha_{i}} u_{i}\right|_{h, g_{\mathbb{C}}} \leq C_{82}^{\prime} \exp \left(-\epsilon_{82}^{\prime} d\right)\left|u_{i}\right|_{h}$. Then, we obtain (7) from Proposition 2.10 and our choice of $u_{i}$. We obtain (8) from the estimate for the curvature $\bar{\partial}\left(\bar{H}^{-1} \partial \bar{H}\right)$ and $(7)$.

Lemma 2.14 Take any $\ell=\left(\ell_{1}, \ell_{2}\right) \in\left(\mathbb{Z}_{\geq 0} \times \mathbb{Z}_{\geq 0}\right) \backslash\{(0,0)\}$ and $R_{4}<R_{3}$. We also take any $p>1$. Then, we have positive constants $C_{83, \ell, p}$ and $\epsilon_{83, \ell, p}$ depending only on $R, R_{1}, R_{2}, R_{3}, R_{4}, r, C_{10}$, $\ell$ and p, such that the following holds:

$$
\left\|\partial_{z}^{\ell_{1}} \partial_{\bar{z}}^{\ell_{2}} H_{\mid \Delta\left(R_{4}\right)}\right\|_{g_{\mathbb{C}}, L^{p}} \leq C_{83, \ell, p} \exp \left(-\epsilon_{83, \ell, p} d\right) .
$$

Proof The proof is given in a standard inductive argument using the Hitchin equation and the elliptic regularity. Because $\Theta_{i i}$ in the Hitchin equation can be large, we give a rather detailed argument.

Let us consider the case $\boldsymbol{\ell}=(2,0)$. By (7) and (8), we have positive constants $C_{84}$ and $\epsilon_{84}$ such that $\left\|\partial_{z} H_{\mid \Delta\left(R_{4}\right)}\right\|_{L_{1}^{p}} \leq C_{84} \exp \left(-\epsilon_{84} d\right)$. So, we obtain $\left\|\partial_{z} \partial_{z} H_{\mid \Delta\left(R_{4}\right)}\right\|_{L^{p}} \leq C_{85} \exp \left(-\epsilon_{85} d\right)$ for some $C_{85}>0$ and $\epsilon_{85}>0$. Similarly, we can obtain the estimate for $\left\|\partial_{\bar{z}} \partial_{\bar{z}} H_{\Delta\left(R_{4}\right)}\right\|_{L^{p}}$. 
Suppose the claim has already been proved for any $R_{4}$ and $p$ if $\ell_{1}+\ell_{2}<k$. We consider $\ell=\left(\ell_{1}, \ell_{2}\right)$ satisfying $\ell_{1}+\ell_{2}=k$ and $\ell_{i}>0$. The Hitchin equation is described as follows:

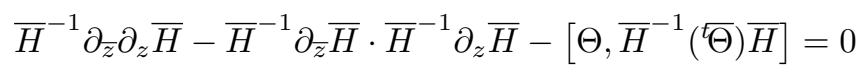

For each $\ell$, we have $C_{86, \ell}$ depending on $C_{10}$ and $\ell$, such that the following holds:

$$
\left|\partial_{z}^{\ell} \Theta_{i i}\right|=\left|\partial_{\bar{z}}^{\ell} \bar{\Theta}_{i i}\right| \leq C_{86, \ell} d
$$

Applying $\partial_{z}^{\ell_{1}-1} \partial_{\bar{z}}^{\ell_{2}-1}$ to (9), we obtain the following:

$$
\bar{H}^{-1} \partial_{z}^{\ell_{1}} \partial_{\bar{z}}^{\ell_{2}} \bar{H}-\left[\partial_{z}^{\ell_{1}-1} \Theta, \bar{H}^{-1} \overline{\left(\partial_{z}^{\ell_{2}-1} \Theta\right)} \bar{H}\right]+G=0
$$

Here, $G$ is expressed as a linear combination of terms which contains derivatives of $\bar{H}$. Hence, we have $\left\|G_{\mid \Delta\left(R_{4}\right)}\right\|_{L^{p}} \leq C_{87} \exp \left(-\epsilon_{87} d\right)$. We also have $\left\|\left[\partial_{z}^{\ell_{1}-1} \Theta, \bar{H}^{-1} \overline{t_{\left(z^{\ell_{2}-1} \Theta\right)}} \bar{H}\right]_{\mid \Delta\left(R_{4}\right)}\right\|_{L^{p}} \leq C_{88} \exp \left(-\epsilon_{88} d\right)$, by (6) and (9). Hence, we obtain the desired estimate if $\ell_{1}+\ell_{2}=k$ and $\ell_{i} \neq 0$. We can deal with the cases $\ell=(k, 0),(0, k)$ by using the elliptic regularity. Then, by an inductive argument, we can obtain the claim of Lemma 2.14 .

Corollary 2.15 Take any $\boldsymbol{\ell}=\left(\ell_{1}, \ell_{2}\right) \in\left(\mathbb{Z}_{\geq 0} \times \mathbb{Z}_{\geq 0}\right) \backslash\{(0,0)\}$ and $R_{4}<R_{3}$. Then, we have positive constants $C_{90, \ell}$ and $\epsilon_{90, \ell}$ depending only on $R, R_{1}, R_{2}, R_{3}, R_{4}, r, C_{10}$ and $\ell$ such that the following holds:

$$
\sup _{\Delta\left(R_{4}\right)}\left|\partial_{z}^{\ell_{1}} \partial_{\bar{z}}^{\ell_{2}} H\right| \leq C_{90, \ell} \exp \left(-\epsilon_{90, \ell} d\right)
$$

\subsection{Hitchin WKB-problem}

\subsubsection{Preliminary}

Let $V$ be an $r$-dimensional complex vector space. For Hermitian metrics $h_{j}(j=1,2)$, we can take a base $e_{1}, \ldots, e_{r}$ of $V$ which is orthogonal with respect to both $h_{1}$ and $h_{2}$. We have the real numbers $\alpha_{i}$ determined by $\kappa_{i}:=\log \left|e_{i}\right|_{h_{2}}-\log \left|e_{i}\right|_{h_{1}}$. We impose $\kappa_{1} \geq \kappa_{2} \geq \cdots \geq \kappa_{r}$. Then, we set

$$
\vec{d}\left(h_{1}, h_{2}\right):=\left(\kappa_{1}, \ldots, \kappa_{r}\right) \in \mathbb{R}^{r} .
$$

Let $V_{j}(j=1,2)$ be $r$-dimensional complex vector spaces with Hermitian metrics $h_{j}$. Let $f: V_{1} \longrightarrow V_{2}$ be a linear isomorphism. We define the Hermitian metric $f^{*} h_{2}$ on $V_{1}$ by $f^{*} h_{2}(u, v)=h_{2}(f(u), f(v))$. We recall the following lemma from [7], which can be easily proved.

Lemma 2.16 We have orthonormal frames $\boldsymbol{q}(s)=\left(q_{1}(s), \ldots, q_{r}(s)\right)(s=0,1)$ on $V_{s}$ such that $f\left(q_{j}(0)\right)=$ $e^{\beta_{j}} q_{j}(1)(j=1, \ldots, r)$, where $\beta_{j}$ are real numbers satisfying $\beta_{1} \geq \beta_{2} \geq \cdots \geq \beta_{r}$. In this case, we have

$$
\vec{d}\left(h_{1}, f^{*} h_{2}\right)=\left(\beta_{1}, \ldots, \beta_{r}\right) .
$$

We set $\|f\|_{\text {op }}:=\sup \left\{\left.|f(u)|_{h_{2}}\left|u \in V_{1},\right| u\right|_{h_{1}}=1\right\}$. By Lemma 2.16, we have $\beta_{1}=\log \|f\|_{\text {op }}$. We also have

$$
\sum_{j=1}^{k} \beta_{j}=\log \left\|\bigwedge^{k} f\right\|_{\mathrm{op}}
$$

where $\bigwedge^{k} f: \bigwedge^{k} V_{1} \longrightarrow \bigwedge^{k} V_{2}$ are the induced morphisms. Hence, we have the following formula, as noted in [7]:

$$
\beta_{k}=\log \left\|\bigwedge^{k} f\right\|_{\mathrm{op}}-\log \left\|\bigwedge^{k-1} f\right\|_{\mathrm{op}}
$$




\subsubsection{Hitchin WKB-problem}

Let $X$ be any complex curve. Let $\phi_{i}(i=1, \ldots, r)$ be holomorphic 1 -forms on $X$. Let $[0,1]:=\{s \in \mathbb{R} \mid 0 \leq s \leq 1\}$ be the closed interval. Let $\gamma:[0,1] \longrightarrow X$ be a $C^{1}$-map. Suppose that it is a non-critical path in the sense of [7], i.e., the following holds:

- At any point $s \in[0,1]$, we have $\gamma^{*} \operatorname{Re}\left(\phi_{i}\right)_{s} \neq \gamma^{*} \operatorname{Re}\left(\phi_{j}\right)_{s}(i \neq j)$.

We have the expression $\gamma^{*}\left(\phi_{i}\right)_{s}=a_{i}(s) d s$ for some $C^{\infty}$-functions $a_{i}:[0,1] \longrightarrow \mathbb{C}$. We may assume $\operatorname{Re} a_{i}(s)<$ $\operatorname{Re} a_{j}(s)(i<j)$ for any $s$. We set

$$
\alpha_{i}:=-\int_{0}^{1} \operatorname{Re}\left(a_{i}\right) d s
$$

We have $\alpha_{1}>\alpha_{2}>\cdots>\alpha_{r}$.

Let $\left(E, \bar{\partial}_{E}, \theta, h\right)$ be a harmonic bundle of rank $r$ on $X$. We suppose the following.

- We have the decomposition $\left(E, \bar{\partial}_{E}, \theta\right)=\bigoplus_{i=1}^{r}\left(E_{i}, \bar{\partial}_{E_{i}}, t \phi_{i} \operatorname{id}_{E_{i}}\right)$ for some $t>0$, where rank $E_{i}=1$.

We have the associated flat connection $\mathbb{D}^{1}=\bar{\partial}_{E}+\partial_{E}+\theta+\theta^{\dagger}$ on $E$. Let $\Pi_{\gamma}: E_{\mid \gamma(0)} \longrightarrow E_{\mid \gamma(1)}$ be the parallel transport of $\mathbb{D}^{1}$ along $\gamma$. We have the metrics $h_{\gamma(0)}$ on the fibers $E_{\mid \gamma(\kappa)}(\kappa=0,1)$ induced by $h$. We obtain the metric $\Pi_{\gamma}^{*} h_{\gamma(1)}$ on $E_{\mid \gamma(0)}$ induced by $h_{\gamma(1)}$ and $\Pi_{\gamma}$.

The following theorem was conjectured in [7], and some cases were verified in [3].

Theorem 2.17 There exist positive constants $t_{0}, \epsilon_{0}$ and $C_{0}$, which may depend only on $X, \phi_{1}, \ldots, \phi_{r}$ and $\gamma$, such that the following holds if $t \geq t_{0}$ :

$$
\left|\frac{1}{t} \vec{d}\left(h_{\gamma(0)}, \Pi_{\gamma}^{*} h_{\gamma(1)}\right)-\left(2 \alpha_{1}, \ldots, 2 \alpha_{r}\right)\right| \leq C_{0} \exp \left(-\epsilon_{0} t\right)
$$

Proof In the following, the constants $C_{i}$ and $\epsilon_{i}$ may depend only on $X, \phi_{1}, \ldots, \phi_{r}$ and $\gamma$, unless otherwise specified.

We can take finite points $s_{0}=0<s_{1}<\cdots<s_{N-1}<s_{N}=1$ and coordinate neighbourhoods $\left(U_{k}, z_{k}\right)$ around $\gamma\left(s_{k}\right)$ such that the following holds:

- We can take $R_{k}>0(k=0, \ldots, N)$ such that $U_{k}$ contains the disc $\Delta_{k}\left(R_{k}\right):=\left\{\left|z_{k}\right|<R_{k}\right\}$ and that $\Delta_{k}\left(R_{k} / 2\right):=\left\{\left|z_{k}\right|<R_{k} / 2\right\}(k=0, \ldots, N)$ give a covering of $\gamma([0,1])$.

- We have the expressions $\phi_{i \mid U_{k}}=f_{k, i} d z_{k}$. Set $d_{k}:=\min \left\{\left|f_{k, i}(0)-f_{k, j}(0)\right| \mid i \neq j\right\}$. Then, we have $\left|f_{k, i}\left(z_{k}\right)-f_{k, i}(0)\right| \leq d_{k} / 100$ on $\Delta_{k}\left(R_{k}\right)$.

If $t_{0}$ is sufficiently large, we have $t_{0} d_{k} \geq 1$ for any $k$. Then, Assumption 2.2 is satisfied for the harmonic bundles $\left(E, \bar{\partial}_{E}, \theta, h\right)_{\mid \Delta_{k}\left(R_{k}\right)}$ if $t \geq t_{0}$. So, we can apply the results in $\S 2.1-2.2$ to each $\left(E, \bar{\partial}_{E}, \theta, h\right)_{\mid \Delta_{k}\left(R_{k}\right)}$.

Let $h_{E_{i}}$ be the restriction of $h$ to $E_{i}$. Let $\nabla_{i}$ be the Chern connection of $\left(E_{i}, \bar{\partial}_{E_{i}}, h_{E_{i}}\right)$, and we have the following connection on $E$ :

$$
\mathbb{D}_{0}^{1}=\bigoplus_{i=1}^{r}\left(\nabla_{i}+t\left(\phi_{i}+\overline{\phi_{i}}\right) \operatorname{id}_{E_{i}}\right)
$$

We fix a Kähler metric $g_{X}$ of $X$. By Corollary 2.13, we have the following estimate on the union of $\Delta_{k}\left(R_{k} / 2\right)$ with respect to $h$ and $g_{X}$ for some positive constants $C_{1}$ and $\epsilon_{1}$ :

$$
\left|\mathbb{D}^{1}-\mathbb{D}_{0}^{1}\right|_{h, g_{X}} \leq C_{1} \exp \left(-\epsilon_{1} t\right)
$$

We have the vector bundle $\gamma^{*} E=\bigoplus_{i=1}^{r} \gamma^{*} E_{i}$ with the metric $\gamma^{*} h$ and the connections $\gamma^{*} \mathbb{D}^{1}$ and $\gamma^{*} \mathbb{D}_{0}^{1}$. We take any orthonormal frames $u_{i}$ of $\gamma^{*} E_{i}$ such that $\gamma^{*} \nabla_{i} u_{i}=0$. They give a frame $\boldsymbol{u}=\left(u_{1}, \ldots, u_{r}\right)$ of $\gamma^{*} E$. We have the following estimates for some positive constants $C_{2}$ and $\epsilon_{2}$ :

$$
\left|\gamma^{*} h\left(u_{i}, u_{j}\right)\right| \leq C_{2} \exp \left(-\epsilon_{2} t\right), \quad(i \neq j) .
$$


The connection $\gamma^{*} \mathbb{D}_{0}^{1}$ is represented by the diagonal matrix $A d s$ with respect to the frame $\boldsymbol{u}$, where the $(i, i)$ entry of $A$ is $2 t \operatorname{Re} a_{i}(s)$. Hence, we have

$$
\gamma^{*} \mathbb{D}^{1} \boldsymbol{u}=\boldsymbol{u}\left(A(s)+B_{0}(s)+B_{1}(s)\right) d s
$$

where $B_{m}(s)(m=0,1)$ are $r$-square matrix valued $C^{\infty}$-functions such that (i) $B_{0}(s)_{i j}=0(i \neq j)$ and $B_{1}(s)_{i j}=0(i=j)$, (ii) there exist $\epsilon_{3}>0$ and $C_{3}>0$ such that

$$
\left|B_{m}(s)\right| \leq C_{3} \exp \left(-\epsilon_{3} t\right) \quad(m=0,1) .
$$

We may assume that $C_{3} \exp \left(-\epsilon_{3} t\right)$ is sufficiently small for any $t \geq t_{0}$ if $t_{0}$ is sufficiently large. Then, applying Corollary 2.19 in $\S 2.4$ below, we have a $C^{1}$-function $G:[0,1] \longrightarrow M_{r}(\mathbb{C})$ and a $C^{0}$-function $H:[0,1] \longrightarrow M_{r}(\mathbb{C})$ such that the following holds for some positive constants $C_{4}$ and $\epsilon_{4}$ :

- The $C^{1}$-norm of $G$ is dominated by $C_{4} \exp \left(-\epsilon_{4} t\right)$.

- $H(s)$ are diagonal, and the $C^{0}$-norm of $H$ is dominated by $C_{4} \exp \left(-\epsilon_{4} t\right)$.

- The connection $\gamma^{*} \mathbb{D}^{1}$ is represented by $\left(A+B_{0}+H\right) d s$ with respect to the frame $\boldsymbol{v}:=\boldsymbol{u}(I+G)$, where $I$ denotes the $r$-square identity matrix.

The frame $\boldsymbol{v} \exp \left(-\int_{0}^{s}\left(A+B_{0}+H\right) d \tau\right)$ of $\gamma^{*} E$ is flat with respect to $\gamma^{*} \mathbb{D}^{1}$. The parallel transport $\Pi_{\gamma}$ of $\gamma^{*} \mathbb{D}^{1}$ from $E_{\gamma(0)}$ to $E_{\gamma(1)}$ is represented by the following matrix with respect to $\boldsymbol{u}(0)$ and $\boldsymbol{u}(1)$ :

$$
(I+G(1)) \exp \left(-\int_{0}^{1}\left(A+B_{0}+H\right) d \tau\right)(I+G(0))^{-1}
$$

Here, $I$ denotes the $r$-square identity matrix.

Let $\boldsymbol{p}(s)=\left(p_{1}(s), \ldots, p_{r}(s)\right)(s=0,1)$ be the orthonormal frames of $E_{\mid \gamma(s)}$, induced by the frames $\boldsymbol{u}(s)$ and the Gram-Schmidt process. We have $\boldsymbol{p}(s)=\boldsymbol{u}(s) \cdot(I+K(s))$, where we have positive constants $C_{5}$ and $\epsilon_{5}$ such that $|K(s)| \leq C_{5} \exp \left(-\epsilon_{5} t\right)$. Let $L(s)(s=0,1)$ be determined by $L(s)=(I+K(s))(I+G(s))-I$. Then, $\Pi_{\gamma}$ is represented by the following matrix with respect to the orthonormal bases $\boldsymbol{p}(0)$ and $\boldsymbol{p}(1)$ :

$$
Z_{\gamma}:=(I+L(1)) \exp \left(-\int_{0}^{1}\left(A+B_{0}+H\right) d \tau\right)(I+L(0))^{-1}
$$

For any $r$-square matrix $Y$, we set

$$
\|Y\|_{\mathrm{op}}:=\sup \left\{|Y \boldsymbol{v}|\left|\boldsymbol{v} \in \mathbb{C}^{r},\right| \boldsymbol{v} \mid=1\right\} .
$$

We clearly have $\left\|Y_{1} Y_{2}\right\|_{\mathrm{op}} \leq\left\|Y_{1}\right\|_{\mathrm{op}}\left\|Y_{2}\right\|_{\mathrm{op}}$. So, we have positive constants $C_{6}$ and $\epsilon_{6}$, such that the following holds:

$$
\log \left\|Z_{\gamma}\right\|_{\text {op }} \leq \log \left\|\exp \left(-\int_{0}^{1}\left(A+B_{0}+H\right) d \tau\right)\right\|_{\text {op }}+\rho_{1}, \quad\left|\rho_{1}\right| \leq C_{6} \exp \left(-\epsilon_{6} t\right)
$$

We also have positive constants $C_{7}$ and $\epsilon_{7}$, such that the following holds:

$$
\log \left\|\exp \left(-\int_{0}^{1}\left(A+B_{0}+H\right) d \tau\right)\right\|_{\text {op }} \leq 2 t \alpha_{1}+\rho_{2}, \quad\left|\rho_{2}\right| \leq C_{7} \exp \left(-\epsilon_{7} t\right)
$$

We also have positive constants $C_{8}$ and $\epsilon_{8}$, such that the following holds:

$$
\left|\Pi_{\gamma} p_{1}(0)\right|_{h_{\gamma(1)}}=\exp \left(2 t \alpha_{1}\right) \cdot\left(1+\rho_{3}\right), \quad\left|\rho_{3}\right| \leq C_{8} \exp \left(-\epsilon_{8} t\right)
$$

Hence, we have positive constants $C_{9}$ and $\epsilon_{9}$, such that the following holds:

$$
\log \left\|Z_{\gamma}\right\|_{\text {op }} \geq 2 t \alpha_{1}+\rho_{4}, \quad\left|\rho_{4}\right| \leq C_{9} \exp \left(-\epsilon_{9} t\right)
$$


Therefore, we have positive constants $C_{10}$ and $\epsilon_{10}$, such that the following holds:

$$
\left|\log \left\|Z_{\gamma}\right\|_{\text {op }}-2 t \alpha_{1}\right| \leq C_{10} \exp \left(-\epsilon_{10} t\right)
$$

By applying the argument to $\bigwedge^{k} Z_{\gamma}$, we obtain positive constants $C_{11}$ and $\epsilon_{11}$, such that the following holds for any $k$ :

$$
\left|\log \left\|\bigwedge^{k} Z_{\gamma}\right\|_{\text {op }}-2 t \sum_{j=1}^{k} \alpha_{j}\right| \leq C_{11} \exp \left(-\epsilon_{11} t\right)
$$

By using (11), we can deduce the claim of the theorem from (12).

\subsection{Appendix: A singular perturbation theory}

We explain a singular perturbation theory which is available in our situation, and which seems slightly different from those in [3] and [7]. Note that we applied Corollary 2.19 in the proof of Theorem 2.17 to find a family of small gauge transforms with which the family of connections $\gamma^{*} \boldsymbol{D}$ on $[0,1]$ are transformed to the connections whose connection matrices are diagonal.

\subsubsection{Preliminary}

We set $[0,1]:=\{s \in \mathbb{R} \mid 0 \leq s \leq 1\}$. For any non-negative integer $\ell$, let $C^{\ell}([0,1])$ denote the space of $\mathbb{C}$-valued $C^{\ell}$-functions on $[0,1]$. We set $\|f\|_{0}:=\sup _{s \in[0,1]}|f(s)|$ for any $f \in C^{0}([0,1])$.

Let $M_{r}(\mathbb{C})$ denote the space of $r$-square matrices. Let $M_{r}(\mathbb{C})_{0}$ denote the space of the $r$-square diagonal matrices, i.e., $M_{r}(\mathbb{C})_{0}=\left\{\left(a_{i j}\right) \in M_{r}(\mathbb{C}) \mid a_{i j}=0(i \neq j)\right\}$. Let $M_{r}(\mathbb{C})_{1}$ denote the set of the off-diagonal matrices, i.e., $M_{r}(\mathbb{C})_{1}:=\left\{\left(a_{i j}\right) \in M_{r}(\mathbb{C}) \mid a_{i j}=0(i=j)\right\}$. We have $M_{r}(\mathbb{C})=M_{r}(\mathbb{C})_{0} \oplus M_{r}(\mathbb{C})_{1}$.

For any non-negative integer $\ell$, let $C^{\ell}\left([0,1], M_{r}(\mathbb{C})\right)$ denote the space of $C^{\ell}$-maps $X:[0,1] \longrightarrow M_{r}(\mathbb{C})$. Similarly, let $C^{\ell}\left([0,1], M_{r}(\mathbb{C})_{\kappa}\right)(\kappa=0,1)$ be the space of $C^{\ell}$-maps $X:[0,1] \longrightarrow M_{r}(\mathbb{C})_{\kappa}$. We set $\|X\|_{0}:=$ $\sup _{i, j}\left\|X_{i j}\right\|_{0}$ for $X \in C^{0}\left([0,1], M_{r}(\mathbb{C})\right)$.

\subsubsection{Statement}

Fix $C_{0}>0$. We consider $a_{j}, b_{j} \in C^{0}([0,1])(j=1, \ldots, r)$ satisfying the following conditions:

- $\operatorname{Re} a_{1}(s)<\operatorname{Re} a_{2}(s)<\cdots<\operatorname{Re} a_{r}(s)$ for any $s$.

- $\left|b_{j}(s)\right| \leq C_{0}$ for any $s$.

For any $t \geq 0$, we put $\alpha_{j}^{t}(s):=t a_{j}(s)+b_{j}(s)$. Let $A^{t}$ denote the $r$-square matrix whose $(j, j)$-entries are $\alpha_{j}^{t}$.

Proposition 2.18 There exist constants $C_{1}>0$ and $\epsilon_{1}>0$, depending only on $C_{0}$, such that the following holds:

- For any $t \geq 0$ and any $B \in C^{0}\left([0,1], M_{r}(\mathbb{C})_{1}\right)$ satisfying $\|B\|_{0} \leq \epsilon_{1}$, we can take $G^{t} \in C^{1}\left([0,1], M_{r}(\mathbb{C})_{1}\right)$ and $H^{t} \in C^{0}\left([0,1], M_{r}(\mathbb{C})_{0}\right)$ such that $(i)\left\|G^{t}\right\|_{0}+\left\|\partial_{s} G^{t}+\left[A^{t}, G^{t}\right]\right\|_{0}+\left\|H^{t}\right\|_{0} \leq C_{1}\|B\|_{0}$, (ii) we have

$$
A^{t}+B=\left(I+G^{t}\right)^{-1}\left(A^{t}+H^{t}\right)\left(I+G^{t}\right)+\left(I+G^{t}\right)^{-1} \partial_{s} G^{t}
$$

Here, I denotes the r-square identity matrix.

We shall prove the proposition in $\$ 2.4 .3-2.4 .4$. Indeed, we shall give a more refined claim (see Corollary 2.23).

We give a reformulation of Proposition 2.18. Recall that when we have a vector bundle $V$ with a connection $\nabla$ and a frame $\boldsymbol{w}=\left(w_{1}, \ldots, w_{m}\right)$, we have the matrix-valued 1-form $A=\left(A_{i j}\right)$ determined by $\nabla w_{j}=\sum A_{i j} w_{i}$, and we describe the relation by $\nabla \boldsymbol{w}=\boldsymbol{w} A$. 
Corollary 2.19 Let $E$ be a $C^{1}$-vector bundle on $[0,1]$ with a frame $\boldsymbol{v}=\left(v_{1}, \ldots, v_{r}\right)$. Let $B \in C^{0}\left([0,1], M_{r}(\mathbb{C})_{1}\right)$ satisfying $\|B\|_{0} \leq \epsilon_{1}$. Take $t \geq 0$. Let $\nabla^{t}$ be the connection on $E$ given as follows:

$$
\nabla^{t} \boldsymbol{v}=\boldsymbol{v}\left(A^{t}(s)+B(s)\right) d s .
$$

Then, we can take $G^{t} \in C^{1}\left([0,1], M_{r}(\mathbb{C})_{1}\right)$ and $H^{t} \in C^{0}\left([0,1], M_{r}(\mathbb{C})_{0}\right)$ such that $(i)\left\|G^{t}\right\|_{0}+\left\|\partial_{s} G^{t}+\left[A^{t}, G^{t}\right]\right\|_{0}+$ $\left\|H^{t}\right\|_{0} \leq C_{1}\|B\|_{0}$, (ii) for the frame $\boldsymbol{u}^{t}=\boldsymbol{v} \cdot\left(I+G^{t}\right)^{-1}$, we have $\nabla^{t} \boldsymbol{u}^{t}=\boldsymbol{u}^{t} \cdot\left(A^{t}+H^{t}\right) d s$. Here, $\epsilon_{1}$ and $C_{1}$ are constants as in Proposition 2.18.

Remark 2.20 In Proposition 2.18, the off-diagonal part B is required to be sufficiently small. It is satisfied in the Hitchin WKB-problem if $t$ is sufficiently large, as observed in Corollary 2.13. However, it is not satisfied in the Riemann-Hilbert WKB-problem [7] even if $t$ is large, in general. But, we may still apply Proposition 2.18 after dividing the path to shorter paths ${ }^{1}$ as did in [7]. It also seems possible to apply Proposition 2.18 to the WKB-problem for family of $\lambda$-connections without going to small paths.

\subsubsection{Some linear maps}

Let $C^{1}\left([0,1], M_{r}(\mathbb{C})_{1}\right)_{\partial}$ denote the subspace of $C^{1}\left([0,1], M_{r}(\mathbb{C})_{1}\right)$ which consists of the functions $X=\left(X_{i j}\right)$ : $[0,1] \longrightarrow M_{r}(\mathbb{C})$ such that $X_{i j}(1)=0(i<j)$ and $X_{i j}(0)=0(i>j)$. We have the linear map $D_{0}^{t}:$ $C^{1}\left([0,1], M_{r}(\mathbb{C})_{1}\right)_{\partial} \longrightarrow C^{0}\left([0,1], M_{r}(\mathbb{C})_{1}\right)$ given by

$$
D_{0}^{t}(X):=\partial_{s} X+\left[A^{t}, X\right], \quad \text { i.e., } D_{0}^{t}(X)_{i j}=\partial_{s} X_{i j}+\left(\alpha_{i}^{t}-\alpha_{j}^{t}\right) X_{i j} .
$$

For $i \neq j$, we put

$$
F_{i, j}^{t}(s):=\int_{0}^{s}\left(\alpha_{i}^{t}(\tau)-\alpha_{j}^{t}(\tau)\right) d \tau .
$$

We have the map $I_{0}^{t}: C^{0}\left([0,1], M_{r}(\mathbb{C})_{1}\right) \longrightarrow C^{1}\left([0,1], M_{r}(\mathbb{C})_{1}\right)_{\partial}$ given as follows:

$$
I_{0}^{t}(X)_{i, j}:= \begin{cases}\int_{1}^{s} \exp \left(-F_{i, j}^{t}(s)+F_{i, j}^{t}(\tau)\right) X_{i, j}(\tau) d \tau & (i<j) \\ \int_{0}^{s} \exp \left(-F_{i, j}^{t}(s)+F_{i, j}^{t}(\tau)\right) X_{i, j}(\tau) d \tau & (i>j)\end{cases}
$$

Together with the identity map on $C^{0}\left([0,1], M_{r}(\mathbb{C})_{0}\right)$, we also obtain the following maps:

$$
\begin{gathered}
I_{0}^{t}: C^{0}\left([0,1], M_{r}(\mathbb{C})_{0}\right) \oplus C^{0}\left([0,1], M_{r}(\mathbb{C})_{1}\right) \longrightarrow C^{0}\left([0,1], M_{r}(\mathbb{C})_{0}\right) \oplus C^{1}\left([0,1], M_{r}(\mathbb{C})_{1}\right)_{\partial} \\
D_{0}^{t}: C^{0}\left([0,1], M_{r}(\mathbb{C})_{0}\right) \oplus C^{1}\left([0,1], M_{r}(\mathbb{C})_{1}\right)_{\partial} \longrightarrow C^{0}\left([0,1], M_{r}(\mathbb{C})_{0}\right) \oplus C^{0}\left([0,1], M_{r}(\mathbb{C})_{1}\right)
\end{gathered}
$$

Then, $I_{0}^{t}$ and $D_{0}^{t}$ are mutually inverse.

Lemma 2.21 There exists a constant $K_{1}>0$, depending only on $C_{0}$, such that the following holds for any $t \geq 0$ and for any $(Z, W) \in C^{0}\left([0,1], M_{r}(\mathbb{C})_{0}\right) \oplus C^{0}\left([0,1], M_{r}(\mathbb{C})_{1}\right)$ :

$$
\left\|I_{0}^{t}(Z, W)\right\|_{0} \leq K_{1}\|(Z, W)\|_{0} .
$$

Proof The estimate for $(i, i)$-entries are obvious by the construction. Let us consider the estimate for $(i, j)$ entries $(i \neq j)$. We set $Q_{i, j}^{t}(s):=t \int_{0}^{s}\left(a_{i}(\tau)-a_{j}(\tau)\right) d \tau$ and $R_{i, j}(s):=\int_{0}^{s}\left(b_{i}(\tau)-b_{j}(\tau)\right) d \tau$. We have $F_{i, j}^{t}=$ $Q_{i, j}^{t}+R_{i, j}$. Because $\operatorname{Re}\left(a_{k}\right)<\operatorname{Re}\left(a_{\ell}\right)(k<\ell)$, we have the following for $s_{1} \leq s_{2}$ :

$$
\operatorname{Re} Q_{i, j}^{t}\left(s_{1}\right)-\operatorname{Re} Q_{i, j}^{t}\left(s_{2}\right) \geq 0(i<j), \quad \operatorname{Re} Q_{i, j}^{t}\left(s_{1}\right)-\operatorname{Re} Q_{i, j}^{t}\left(s_{2}\right) \leq 0(i>j) .
$$

Hence, we have a constant $K_{1}^{\prime}$, depending only on $C_{0}$, such that $\left|\exp \left(-F_{i, j}^{t}(s)+F_{i, j}^{t}(\tau)\right)\right| \leq K_{1}^{\prime}$ holds in the cases (i) $s \leq \tau$ and $i<j$, (ii) $s \geq \tau$ and $i>j$. Then, we obtain the following in the case $i<j$ :

$$
\left|I_{0}^{t}(X)_{i, j}\right| \leq \int_{s}^{1}\left|\exp \left(-F_{i, j}^{t}(s)+F_{i, j}^{t}(\tau)\right) X_{i, j}(\tau)\right| d \tau \leq K_{1}^{\prime} \int_{s}^{1}\left|X_{i, j}(\tau)\right| d \tau \leq K_{1}^{\prime}\left\|X_{i, j}\right\|_{0}
$$

Hence, we obtain $\left\|I_{0}^{t}(X)_{i, j}\right\|_{0} \leq K_{1}^{\prime}\left\|X_{i, j}\right\|_{0}$ in the case $i<j$. Similarly, we obtain $\left\|I_{0}^{t}(X)_{i, j}\right\|_{0} \leq K_{1}^{\prime}\left\|X_{i, j}\right\|_{0}$ in the case $i>j$.

\footnotetext{
${ }^{1}$ This was remarked by C. Simpson.
} 


\subsubsection{Proof of Proposition 2.18}

We take a small number $\epsilon>0$. We set

$$
\mathcal{H}_{\epsilon}:=\left\{H \in C^{0}\left([0,1], M_{r}(\mathbb{C})_{0}\right) \mid\|H\|_{0} \leq \epsilon\right\}, \quad \mathcal{G}_{\epsilon}^{t}:=\left\{G \in C^{1}\left([0,1], M_{r}(\mathbb{C})_{1}\right)_{\partial} \mid\left\|D_{0}^{t} G\right\|_{0} \leq \epsilon\right\} .
$$

Note that $\|G\|_{0} \leq K_{1}\left\|D_{0}^{t} G\right\|_{0}$ for any $G \in \mathcal{G}_{\epsilon}^{t}$.

Let $I \in M_{r}(\mathbb{C})$ denote the identity matrix. If $\epsilon K_{1}<1 / 2$, then $(I+G)(s)$ are invertible for any $G \in \mathcal{G}_{\epsilon}^{t}$. So, we have the maps $J^{t}: \mathcal{H}_{\epsilon} \times \mathcal{G}_{\epsilon}^{t} \longrightarrow C^{0}\left([0,1], M_{r}(\mathbb{C})_{0}\right) \oplus C^{0}\left([0,1], M_{r}(\mathbb{C})_{1}\right)$ given by

$$
J^{t}(H, G):=(I+G)^{-1}\left(A^{t}+H\right)(I+G)+(I+G)^{-1} \partial_{s} G-A^{t} .
$$

Let $T_{(H, G)} J^{t}$ denote the derivative at $(H, G)$. We have $T_{(0,0)} J^{t}(X, Y)=X+D_{0}^{t} Y$. More generally, we have

$$
\begin{array}{r}
T_{(H, G)} J^{t}(X, Y)=(I+G)^{-1} X(I+G)+(I+G)^{-1}\left(A^{t}+H\right) Y-(I+G)^{-1} Y(I+G)^{-1}\left(A^{t}+H\right)(I+G) \\
-(I+G)^{-1} Y(I+G)^{-1} \partial_{s} G+(I+G)^{-1} \partial_{s} Y
\end{array}
$$

We regard $T_{(H, G)} J^{t}$ as maps $C^{0}\left([0,1], M_{r}(\mathbb{C})_{0}\right) \oplus C^{1}\left([0,1], M_{r}(\mathbb{C})_{1}\right)_{\partial} \longrightarrow C^{0}\left([0,1], M_{r}(\mathbb{C})_{0}\right) \oplus C^{0}\left([0,1], M_{r}(\mathbb{C})_{1}\right)$. We obtain the following family of endomorphisms on $C^{0}\left([0,1], M_{r}(\mathbb{C})_{0}\right) \oplus C^{0}\left([0,1], M_{r}(\mathbb{C})_{1}\right)$ :

$$
T_{(H, G)} J^{t} \circ\left(T_{(0,0)} J^{t}\right)^{-1}, \quad\left((H, G) \in \mathcal{H}_{\epsilon} \times \mathcal{G}_{\epsilon}^{t}\right) .
$$

Lemma 2.22 There exists a constant $C_{2}>0$, depending only on $C_{0}$, such that the following holds for any $t \geq 0$ and any $(Z, W) \in C^{0}\left([0,1], M_{r}(\mathbb{C})_{0}\right) \oplus C^{0}\left([0,1], M_{r}(\mathbb{C})_{1}\right)$ :

$$
\left\|T_{(H, G)} J^{t} \circ\left(T_{(0,0)} J^{t}\right)^{-1}(Z, W)-(Z, W)\right\|_{0} \leq C_{2}\left(\|H\|_{0}+\left\|D_{0}^{t} G\right\|_{0}\right)\left(\|Z\|_{0}+\|W\|_{0}\right)
$$

Proof We consider

$$
(I+G)^{-1}\left(A^{t}\right) Y-(I+G)^{-1} Y(I+G)^{-1}\left(A^{t}\right)(I+G)-(I+G)^{-1} Y(I+G)^{-1} \partial_{s} G+(I+G)^{-1} \partial_{s} Y-D_{0}^{t} Y .
$$

We have $(I+G)^{-1} \partial_{s} Y=(I+G)^{-1} D_{0}^{t} Y-(I+G)^{-1}\left[A^{t}, Y\right]$. Hence, (15) is rewritten as

$$
\begin{aligned}
&(I+G)^{-1}\left(A^{t}\right) Y-(I+G)^{-1} Y(I+G)^{-1}\left(A^{t}\right)(I+G)-(I+G)^{-1} Y(I+G)^{-1} \partial_{s} G \\
&-(I+G)^{-1}\left[A^{t}, Y\right]+\left((I+G)^{-1}-I\right) D_{0}^{t} Y
\end{aligned}
$$

It is equal to the following:

$$
\begin{aligned}
&(I+G)^{-1} Y\left(A^{t}\right)-(I+G)^{-1} Y(I+G)^{-1}\left(A^{t}\right)(I+G)-(I+G)^{-1} Y(I+G)^{-1} \partial_{s} G \\
&+\left((I+G)^{-1}-I\right) D_{0}^{t} Y
\end{aligned}
$$

We have the following:

$$
\begin{gathered}
-(I+G)^{-1} Y(I+G)^{-1}\left(A^{t}\right)(I+G)-(I+G)^{-1} Y(I+G)^{-1} \partial_{s} G \\
\quad=-(I+G)^{-1} Y(I+G)^{-1}\left(A^{t}\right)-(I+G)^{-1} Y(I+G)^{-1}\left(\partial_{s} G+\left(A^{t}\right) G\right) \\
=-(I+G)^{-1} Y(I+G)^{-1}\left(A^{t}\right)-(I+G)^{-1} Y(I+G)^{-1} G\left(A^{t}\right)-(I+G)^{-1} Y(I+G)^{-1} D_{0}^{t} G
\end{gathered}
$$

Hence, (17) is equal to the following:

$$
\begin{array}{r}
(I+G)^{-1} Y\left(A^{t}\right)-(I+G)^{-1} Y(I+G)^{-1}\left(A^{t}\right)-(I+G)^{-1} Y(I+G)^{-1} G\left(A^{t}\right) \\
-(I+G)^{-1} Y(I+G)^{-1} D_{0}^{t} G+\left((I+G)^{-1}-I\right) D_{0}^{t} Y \\
=-(I+G)^{-1} Y(I+G)^{-1} D_{0}^{t} G+\left((I+G)^{-1}-I\right) D_{0}^{t} Y
\end{array}
$$


In all, we obtain the following:

$$
\begin{aligned}
T_{(H, G)} J^{t} \circ\left(T_{(0,0)} J^{t}\right)^{-1}(Z, W)-( & Z, W)=-(I+G)^{-1} I_{0}^{t}(W)(I+G)^{-1} D_{0}^{t} G-\left((I+G)^{-1}-I\right) W \\
& +(I+G)^{-1} Z(I+G)-Z+\left[(I+G)^{-1} H(I+G),(I+G)^{-1} I_{0}^{t}(W)\right]
\end{aligned}
$$

Because $\|G\|_{0} \leq K_{1}\left\|D_{0}^{t} G\right\|_{0}$, we have $\left\|(I+G)^{-1}-I\right\|_{0} \leq C_{3}\left\|D_{0}^{t} G\right\|_{0}$ and $\left\|(I+G)^{-1} X(I+G)-X\right\|_{0} \leq$ $C_{4}\left(\left\|D_{0}^{t} G\right\|_{0}\|X\|_{0}\right)$ for positive constants $C_{i}(i=3,4)$ depending only on $C_{0}$. We also have the following for a positive constant $C_{5}$ depending only on $C_{0}$ :

$$
\left\|\left[(I+G)^{-1} H(I+G),(I+G)^{-1} Y\right]\right\|_{0} \leq C_{5}\left(\left\|D_{0}^{t} G\right\|_{0}+\|H\|_{0}\right)\left\|D_{0}^{t} Y\right\|_{0}
$$

Hence, we obtain the claim of the lemma.

Corollary 2.23 There exist positive constants $\epsilon_{10}>0$ and $C_{10}>0$, depending only on $C_{0}$, with the following property:

- For any $t \geq 0$ and any $B \in C^{0}\left([0,1], M_{r}(\mathbb{C})_{1}\right)$ such that $\|B\|_{0} \leq \epsilon_{10}$, we have a unique $\left(H^{t}, G^{t}\right) \in \mathcal{H}_{\epsilon} \times \mathcal{G}_{\epsilon}^{t}$ such that $J^{t}\left(H^{t}, G^{t}\right)=B$ and that $\left\|H^{t}\right\|_{0}+\left\|D_{0}^{t} G^{t}\right\|_{0} \leq C_{10}\|B\|_{0}$.

Proof We set $\overline{\mathcal{G}}_{\epsilon}:=\left\{\bar{G} \in C^{0}\left([0,1], M_{r}(\mathbb{C})_{1}\right) \mid\|\bar{G}\|_{0} \leq \epsilon\right\} \subset C^{0}\left([0,1], M_{r}(\mathbb{C})_{1}\right)$. We have the bijections $I_{0}^{t}: \overline{\mathcal{G}}_{\epsilon} \simeq \mathcal{G}_{\epsilon}^{t}$ for any $t \geq 0$. We consider the maps

$$
\mathcal{F}^{t}:=J^{t} \circ\left(T_{(0,0)} J^{t}\right)^{-1}: \mathcal{H}_{\epsilon} \times \overline{\mathcal{G}}_{\epsilon} \longrightarrow C^{0}\left([0,1], M_{r}(\mathbb{C})_{0}\right) \oplus C^{0}\left([0,1], M_{r}(\mathbb{C})_{1}\right) .
$$

Let $T \mathcal{F}^{t}: C^{0}\left([0,1], M_{r}(\mathbb{C})\right) \longrightarrow C^{0}\left([0,1], M_{r}(\mathbb{C})\right)$ denote the derivative of $\mathcal{F}^{t}$. According to Lemma 2.22 , we have a positive constant $C_{3}$ such that the operator norms of $T_{(H, \bar{G})} \mathcal{F}^{t}-$ id are dominated by $C_{3}\left(\|H\|_{0}+\|\bar{G}\|_{0}\right)$ for any $(H, \bar{G}) \in \mathcal{H}_{\epsilon} \times \overline{\mathcal{G}}_{\epsilon}$. The constant $C_{3}$ may depend only on $C_{0}$, and the estimate is uniform for $t$. By the inverse function theorem (see [8], for instance), there exist positive constants $\epsilon_{10}$ and $C_{10}$, depending only on $C_{0}$, with the following property:

- For any $t \geq 0$ and any $B \in C^{0}\left([0,1], M_{r}(\mathbb{C})_{1}\right)$ such that $\|B\|_{0} \leq \epsilon_{10}$, we have a unique $\left(H^{t}, \bar{G}^{t}\right) \in \mathcal{H}_{\epsilon} \times \overline{\mathcal{G}}_{\epsilon}$ such that $\mathcal{F}^{t}\left(H^{t}, \bar{G}^{t}\right)=B$ and that $\left\|H^{t}\right\|_{0}+\left\|\bar{G}^{t}\right\|_{0} \leq C_{10}\|B\|_{0}$.

By setting $G^{t}:=\left(T_{(0,0)} J^{t}\right)^{-1} \bar{G}^{t}$, we obtain the claim of Corollary 2.23. We also finish the proof of Proposition 2.18 .

\subsection{Appendix: The case of Hermitian-Einstein metrics}

For $R>0$, we set $\Delta(R):=\{z \in \mathbb{C}|| z \mid<R\}$. Let $\left(E, \bar{\partial}_{E}, \theta\right)$ be a Higgs bundle of rank $r$ on $\Delta(R)$. We fix a Hermitian metric $h_{\operatorname{det}(E)}$ of $\operatorname{det}(E)$. Let $h$ be a Hermitian-Einstein metric of $\left(E, \bar{\partial}_{E}, \theta\right)$ such that $\operatorname{det}(h)=$ $h_{\operatorname{det}(E)}$, i.e., $R(h)^{\perp}+\left[\theta, \theta_{h}^{\dagger}\right]=0$, where $R(h)^{\perp}$ denotes the trace-free part of the curvature $R(h)$. We have an obvious generalization of the results in $\S 2.1$ and $\S 2.2$, which we state explicitly in this subsection.

We have a $C^{\infty}$-function $\nu$ such that $\bar{\partial} \partial \nu=R\left(h_{\operatorname{det}(E)}\right) / r$ such that the $L_{2}^{p}$-norm of $\nu$ is dominated by $C_{0, p}\left\|R\left(h_{\operatorname{det}(E)}\right)\right\|_{\infty}(p>1)$, where $\left\|R\left(h_{\operatorname{det}(E)}\right)\right\|_{\infty}$ denotes the sup norm of $R\left(h_{\operatorname{det}(E)}\right)$ with respect to the Euclidean metric, and $C_{0, p}$ denotes the constant depending on $p$ and the radius $R$. Then, the metric $\widetilde{h}=h e^{-\nu}$ is a harmonic metric for the Higgs bundle $\left(E, \bar{\partial}_{E}, \theta\right)$. Note that $h$ and $\widetilde{h}$ induce the same metrics on the vector bundles $\operatorname{End}(E) \otimes \Omega^{p, q}$.

We have the description $\theta=f d z$. Let $M$ be the constant as in $\S 2.1 .1$. We obtain the following from Proposition 2.1.

Corollary 2.24 Fix $0<R_{1}<R$. Then, we have $C_{1}, C_{2}>0$ depending only on $r, R_{1}, R$ such that $|f|_{h} \leq$ $C_{1} M+C_{2}$ holds on $\Delta\left(R_{1}\right)$.

We impose the conditions as in Assumption 2.2. We obtain the following from Proposition 2.3. 
Corollary 2.25 Let $R_{1}$ be as in Proposition 2.1. Fix $0<R_{2}<R_{1}$. There exist positive constants $\epsilon_{0}$ and $C_{11}$ depending only on $R, R_{1}, R_{2}, r$ and $C_{10}$, such that $\left|\rho_{\alpha}\right|_{h} \leq C_{11} \exp \left(-\epsilon_{0} d\right)$ on $\Delta\left(R_{2}\right)$.

We impose the condition that $\operatorname{rank} E_{\alpha}=1$ as in $\S 2.2$. We have $R(\widetilde{h})=R(h)^{\perp}$ and $\theta_{h}^{\dagger}=\theta_{\widetilde{h}}^{\dagger}$. Hence, we obtain the following from Theorem 2.9 .

Corollary 2.26 There exist positive constants $C_{30}$ and $\epsilon_{30}$, depending only on $R, R_{1}, R_{2}, r$ and $C_{10}$ such that $\left|R(h)^{\perp}\right|_{h, g_{\mathbb{C}}}=\left|\left[\theta, \theta_{h}^{\dagger}\right]\right|_{h, g_{\mathbb{C}}} \leq C_{30} \exp \left(-\epsilon_{30} d\right)$ on $\Delta\left(R_{2}\right)$.

Because $h$ and $\widetilde{h}$ give the same connection on $\operatorname{End}(E)$ as the Chern connections, we obtain the following from Proposition 2.10 and Proposition 2.11.

Corollary 2.27 Take $0<R_{3}<R_{2}$. There exist positive constants $\epsilon_{40}$ and $C_{40}$ depending only on $R$, $R_{1}$, $R_{2}, R_{3}, r$ and $C_{10}$ such that $\left|\partial_{E, h} \pi_{\alpha}\right|_{h, g_{\mathbb{C}}}=\left|\bar{\partial}_{E} \pi_{\alpha}^{\dagger}\right|_{h, g_{\mathbb{C}}} \leq C_{40} \exp \left(-\epsilon_{40} d\right)$ and $\left|\bar{\partial}_{E} \pi_{\alpha}^{\prime}\right|_{h, g_{\mathbb{C}}}=\left|\partial_{E, h} \pi_{\alpha}^{\prime}\right|_{h, g_{\mathbb{C}}} \leq$ $C_{40} \exp \left(-\epsilon_{40} d\right)$ on $\Delta\left(R_{3}\right)$.

Let $h_{\alpha}\left(\right.$ resp. $\left.h_{0, \alpha}\right)$ denote the restriction of $h\left(\right.$ resp. $\widetilde{h}$ ) to $L_{\alpha}$. Because $h_{\alpha}=\widetilde{h}_{\alpha} e^{\nu / r}$, we obtain the following from Proposition 2.12.

Corollary 2.28 We have a positive constant $C_{60}$ depending only on $R, R_{1}, R_{2}, R_{3}, r$ and $C_{10}$ such that $\left|R\left(h_{\alpha}\right)-R\left(h_{\operatorname{det}(E)}\right) / r\right|_{h_{\alpha}, g_{\mathbb{C}}} \leq C_{60} \exp \left(-\epsilon_{40} d\right)$ on $\Delta\left(R_{3}\right)$.

We have the projectively flat connection $\mathbb{D}^{1}:=\bar{\partial}_{E}+\partial_{E, h}+\theta+\theta_{h}^{\dagger}$ induced by $h$. We also have the flat connection $\widetilde{\mathbb{D}}^{1}:=\bar{\partial}_{E}+\partial_{E, \widetilde{h}}+\theta+\theta_{\widetilde{h}}^{\dagger}$. They are related as $\mathbb{D}^{1}=\widetilde{\mathbb{D}}^{1}+\partial \nu \cdot \operatorname{id}_{E}$. We have the Chern connections $\bar{\partial}_{\alpha}+\partial_{\alpha, h}$ on $E_{\alpha}$ induced by $h_{\alpha}$. Similarly, we have the Chern connections $\bar{\partial}_{\alpha}+\partial_{\alpha, \widetilde{h}}$ on $E_{\alpha}$ induced by $\widetilde{h}_{\alpha}$. They are related as $\partial_{\alpha, h}=\partial_{\alpha, \widetilde{h}}+\partial \nu \cdot \operatorname{id}_{E_{\alpha}}$. Hence, we obtain the following from Corollary 2.13.

Corollary 2.29 We set $\mathbb{D}_{0}^{1}:=\bigoplus\left(\bar{\partial}_{\alpha}+\partial_{\alpha, h}+\left(g_{\alpha} d z+\overline{g_{\alpha}} d \bar{z}\right) \cdot \mathrm{id}_{E_{\alpha}}\right)$ as in $\S 2.2 .4$. Then, there exists a positive constant $C_{70}$, depending only on $R, R_{1}, R_{2}, R_{3}, r$ and $C_{10}$ such that $\left|\mathbb{D}^{1}-\mathbb{D}_{0}^{1}\right|_{h, g_{\mathbb{C}}} \leq C_{70} \exp \left(-\epsilon_{40} d\right)$ holds on $\Delta\left(R_{3}\right)$.

We also have an obvious generalization of the estimates of the higher derivatives in $\S 2.2 .5$, which we omit to describe.

\section{Local models}

\subsection{Review on unramifiedly good filtered Higgs bundles}

\subsubsection{Filtered bundles on curves}

Let $X$ be a complex curve with a discrete subset $D$. We recall the concept of filtered bundles on $(X, D)[20]$. Let $\mathbb{R}^{D}$ denote the set of maps $D \longrightarrow \mathbb{R}$. Elements of $\mathbb{R}^{D}$ are denoted by $\boldsymbol{a}=\left(a_{P} \mid P \in D\right)$. Let $\mathcal{O}_{X}(* D)$ be the sheaf of meromorphic functions on $X$ whose poles are contained in $D$. Let $\mathcal{E}$ be a locally free $\mathcal{O}_{X}(* D)$-module of rank $r$. A filtered bundle over $\mathcal{E}$ is a family of coherent $\mathcal{O}_{X}$-submodules $\mathcal{P}_{*} \mathcal{E}=\left(\mathcal{P}_{\boldsymbol{a}} \mathcal{E} \subset \mathcal{E} \mid \boldsymbol{a} \in \mathbb{R}^{D}\right)$ with the following property.

- $\mathcal{P}_{\boldsymbol{a}} \mathcal{E}$ are lattices of $\mathcal{E}$, i.e., $\mathcal{P}_{\boldsymbol{a}} \mathcal{E} \otimes_{\mathcal{O}_{X}} \mathcal{O}_{X}(* D)=\mathcal{E}$.

- The stalk of $\mathcal{P}_{\boldsymbol{a}} \mathcal{E}$ at $P \in D$ depends only on $a_{P} \in \mathbb{R}$. We denote it by $\mathcal{P}_{a_{P}}^{P}\left(\mathcal{E}_{P}\right)$.

- We have $\mathcal{P}_{a}^{P}\left(\mathcal{E}_{P}\right) \subset \mathcal{P}_{b}^{P}\left(\mathcal{E}_{P}\right)$ for $a \leq b$. For any $a \in \mathbb{R}$, there exists $\epsilon>0$ such that $\mathcal{P}_{a}^{P}\left(\mathcal{E}_{P}\right)=\mathcal{P}_{a+\epsilon}^{P}\left(\mathcal{E}_{P}\right)$.

- For $n \in \mathbb{Z}$, we have $\mathcal{P}_{a}^{P}\left(\mathcal{E}_{P}\right) \otimes_{\mathcal{O}_{X, P}} \mathcal{O}_{X}(n P)_{P}=\mathcal{P}_{a+n}^{P}\left(\mathcal{E}_{P}\right)$.

Such $\mathcal{P}_{*} \mathcal{E}$ is called a filtered bundle over $\mathcal{E}$ on $(X, D)$. For any $\mathcal{O}_{X}(* D)$-submodule $\mathcal{G} \subset \mathcal{E}$, we have the induced filtered bundle $\mathcal{P}_{*} \mathcal{G}$ over $\mathcal{G}$ given by $\mathcal{P}_{\boldsymbol{a}} \mathcal{G}=\mathcal{P}_{\boldsymbol{a}} \mathcal{E} \cap \mathcal{G}$ in $\mathcal{E}$. 
Parabolic structure Let $\mathcal{P}_{*} \mathcal{E}$ be a filtered bundle on $(X, D)$. Let $\mathbf{0} \in \mathbb{R}^{D}$ denote the element such that the $P$-th component of $\mathbf{0}$ are 0 for any $P \in D$. We have the locally free $\mathcal{O}_{X}$-module $\mathcal{P}_{\mathbf{0}} \mathcal{E}$ on $X$. For each $Q \in X$, let $\mathcal{P}_{0} \mathcal{E}_{\mid Q}$ denote the fiber of the vector bundle $\mathcal{P}_{\mathbf{0}} \mathcal{E}$ over $Q$. For $P \in D$ and for $-1<a \leq 0$, we set

$$
F_{a}^{P}\left(\mathcal{P}_{0} \mathcal{E}_{\mid P}\right):=\operatorname{Im}\left(\mathcal{P}_{a}^{P}\left(\mathcal{E}_{P}\right) \longrightarrow \mathcal{P}_{0} \mathcal{E}_{\mid P}\right) .
$$

In this way, we obtain a filtration $F^{P}$ of $\mathcal{P}_{0} \mathcal{E}_{\mid P}$ indexed by $\{-1<a \leq 0\}$. We have $F_{0}^{P}\left(\mathcal{P}_{0} \mathcal{E}_{\mid P}\right)=\mathcal{P}_{0} \mathcal{E}_{\mid P}$. Note that we have the natural identification $\mathcal{P}_{0} \mathcal{E}_{\mid P} \simeq \mathcal{P}_{0}^{P}\left(\mathcal{E}_{P}\right) / \mathcal{P}_{-1}^{P}\left(\mathcal{E}_{P}\right)$ because $\mathcal{P}_{a+n}^{P}(\mathcal{E})=\mathcal{P}_{a}^{P}(\mathcal{E}) \otimes_{\mathcal{O}_{X, P}} \mathcal{O}_{X, P}(n P)$, and hence we have $F_{-1}^{P}\left(\mathcal{P}_{0} \mathcal{E}_{\mid P}\right)=0$. For any $-1<a \leq 0$, there exists $\epsilon>0$ such that $F_{a}^{P}\left(\mathcal{P}_{0} \mathcal{E}_{\mid P}\right)=F_{a+\epsilon}^{P} \mathcal{P}_{0} \mathcal{E}_{\mid P}$. Such a family of filtrations $\left(F^{P} \mid P \in D\right)$ is called a parabolic structure on the vector bundle $\mathcal{P}_{0} \mathcal{E}$ along $D$. We can easily observe that filtered bundles on $(X, D)$ are equivalent to vector bundles on $X$ with a parabolic structure along $D$.

\subsubsection{Unramifiedly good filtered Higgs bundles}

Let $X$ and $D$ be as in $\S 3.1 .1$. Let $\mathcal{P}_{*} \mathcal{E}$ be a filtered bundle on $(X, D)$. Let $\theta$ be a Higgs field of $\mathcal{E}$, i.e., $\theta$ is a holomorphic section of $\operatorname{End}(\mathcal{E}) \otimes \Omega_{X}^{1}$. The filtered bundle with a Higgs field $\left(\mathcal{P}_{*} \mathcal{E}, \theta\right)$ is called unramifiedly good at $P$, if the following holds.

- We have a finite subset $\mathcal{I}(P) \subset \mathcal{O}_{X}(* D)_{P}$ and a decomposition of the stalk $\mathcal{P}_{a}^{P} \mathcal{E}_{P}=\bigoplus_{\mathfrak{a} \in \mathcal{I}(P)} \mathcal{P}_{a}^{P} \mathcal{E}_{P, \mathfrak{a}}$ such that

$$
(\theta-d \mathfrak{a} \text { id }) \mathcal{P}_{a}^{P} \mathcal{E}_{P, \mathfrak{a}} \subset \mathcal{P}_{a}^{P} \mathcal{E}_{P, \mathfrak{a}} \otimes \Omega_{X}^{1}(\log D)_{P}
$$

Here, $d \mathfrak{a}$ denotes the exterior derivative of $\mathfrak{a} \in \mathcal{I}(P) \subset \mathcal{O}_{X}(* D)_{P}$. We have $\mathcal{P}_{a}^{P} \mathcal{E}_{P, \mathfrak{a}} \subset \mathcal{P}_{b}^{P} \mathcal{E}_{P, \mathfrak{a}}$ for $a \leq b$. The induced map $\mathcal{I}(P) \longrightarrow \mathcal{O}_{X}(* D)_{P} / \mathcal{O}_{X, P}$ is assumed to be injective.

If $\left(\mathcal{P}_{*} \mathcal{E}, \theta\right)$ is unramifiedly good at any $P \in D$, it is called an unramifiedly good filtered Higgs bundle.

Remark 3.1 More generally, a filtered bundle with a Higgs field $\left(\mathcal{P}_{*} \mathcal{E}, \theta\right)$ is called good at $P$ if we have a neighbourhood $X_{P}$ of $P$, a Galois covering map $X_{P}^{\prime} \longrightarrow X_{P}$ ramified along $P$, and an unramifiedly good filtered Higgs bundle $\left(\mathcal{P}_{*}^{P} \mathcal{E}^{\prime}, \theta^{\prime}\right)$ on $\left(X_{P}^{\prime}, \pi^{-1}(P)\right)$ such that $\left(\mathcal{P}_{*} \mathcal{E}, \theta\right)_{\mid X_{P}}$ is the descent of $\left(\mathcal{P}_{*}^{P} \mathcal{E}^{\prime}, \theta^{\prime}\right)$. In this paper, we consider only unramified ones.

\subsubsection{Unramified wild harmonic bundles and the associated unramifiedly good filtered Higgs bundles}

Let $X$ and $D$ be as in $\S 3.1 .1$. Let $\left(E, \bar{\partial}_{E}, \theta, h\right)$ be a harmonic bundle on $X \backslash D$. It is called wild and unramified over $(X, D)$ if the following holds for any $P \in D$.

- Let $\left(U_{P}, z\right)$ be a holomorphic coordinate neighbourhood of $P$ in $X$ with $z(P)=0$. Then, we have a finite subset $\mathcal{I}(P) \subset z^{-1} \mathbb{C}\left[z^{-1}\right]$ and a decomposition

$$
\left(E, \bar{\partial}_{E}, \theta\right)_{\mid U_{P} \backslash\{P\}}=\bigoplus_{\mathfrak{a} \in \mathcal{I}(P)}\left(E_{\mathfrak{a}}, \bar{\partial}_{E_{\mathfrak{a}}}, \theta_{\mathfrak{a}}\right),
$$

and the coefficients $a_{\mathfrak{a}, j}(z)$ of the characteristic polynomials $\operatorname{det}\left(t \mathrm{id}-g_{\mathfrak{a}}\right)=\sum a_{\mathfrak{a}, j}(z) t^{j}$ are holomorphic at $z=0$, where $g_{\mathfrak{a}} \in \operatorname{End}\left(E_{\mathfrak{a}}\right)$ are determined by $\theta_{\mathfrak{a}}-d \mathfrak{a} \operatorname{id}_{E_{\mathfrak{a}}}=g_{\mathfrak{a}} d z / z$.

A unramified wild harmonic bundle is called tame if $\mathcal{I}(P)=\{0\}$ in $\mathcal{O}_{X}(* D)_{P} / \mathcal{O}_{X, P}$ at each $P \in D$.

The tame case of the following proposition was established in [20], and generalized to the wild case in [16].

Proposition 3.2 Let $\left(E, \bar{\partial}_{E}, \theta, h\right)$ be an unramified wild harmonic bundle on $(X, D)$. Then, we have an unramifiedly good filtered Higgs bundle $\left(\mathcal{P}_{*}^{h} E, \theta\right)$ on $(X, D)$ with the following property.

- We have $\left(\mathcal{P}_{\boldsymbol{a}}^{h} E, \theta\right)_{\mid X \backslash D}=(E, \theta)$ for any $\boldsymbol{a} \in \mathbb{R}^{D}$.

- Let $U$ be any open subset of $X$. Let $f$ be a holomorphic section of $E$ on $U \backslash D$. Then, $f$ is a section of $\mathcal{P}_{a}^{h} E$ on $U$ if and only if $|f|_{h}=O\left(\left|z_{P}\right|^{-a_{P}-\epsilon}\right)$ for any $\epsilon>0$ around each $P \in U \cap D$, where $z_{P}$ denotes a holomorphic coordinate around $P$ with $z_{P}(P)=0$.

In this situation, $h$ is called adapted to the filtered bundle $\mathcal{P}_{*}^{h} E$. 


\subsubsection{Filtered Higgs bundles on compact Riemann surfaces}

Suppose that $X$ is a compact connected Riemann surface with a finite subset $D$. Let $\left(\mathcal{P}_{*} \mathcal{E}, \theta\right)$ be an unramifiedly good filtered Higgs bundle on $(X, D)$. Let $\mathcal{G} \subset \mathcal{E}$ be any locally free $\mathcal{O}_{X}(* D)$-submodule. We set $\mathcal{P}_{\boldsymbol{a}} \mathcal{G}:=\mathcal{P}_{\boldsymbol{a}} \mathcal{E} \cap \mathcal{G}$ in $\mathcal{E}$ for any $\boldsymbol{a} \in \mathbb{R}^{D}$, and then we obtain a filtered bundle $\mathcal{P}_{*} \mathcal{G}$ over $\mathcal{G}$. The degree of $\mathcal{P}_{*} \mathcal{G}$ is defined as follows $[9,10]$ for any $\boldsymbol{b} \in \mathbb{R}^{D}$ :

$$
\operatorname{deg}\left(\mathcal{P}_{*} \mathcal{G}\right)=\operatorname{deg}\left(\mathcal{P}_{\boldsymbol{b}} \mathcal{G}\right)-\sum_{P \in D} \sum_{b_{P}-1<a \leq b_{P}} a \cdot \operatorname{dim}_{\mathbb{C}}\left(\mathcal{P}_{a}^{P}\left(\mathcal{G}_{P}\right) / \mathcal{P}_{<a}^{P}\left(\mathcal{G}_{P}\right)\right)
$$

Here, we set $\mathcal{P}_{<a}^{P}\left(\mathcal{G}_{P}\right)=\bigcup_{c<a} \mathcal{P}_{c}^{P}\left(\mathcal{G}_{P}\right)$, and we regard $\mathcal{P}_{a}^{P}\left(\mathcal{G}_{P}\right) / \mathcal{P}_{<a}^{P}\left(\mathcal{G}_{P}\right)$ as finite dimensional $\mathbb{C}$-vector spaces in a natural way. It is easy to see that the right hand side of (21) is independent of the choice of $\boldsymbol{b}$. The unramifiedly good filtered Higgs bundle $\left(\mathcal{P}_{*} \mathcal{E}, \theta\right)$ is called stable (resp. semistable) if the following inequality holds for any locally free $\mathcal{O}_{X}(* D)$-submodule $\mathcal{G} \subset \mathcal{E}$ with (i) $\theta \mathcal{G} \subset \mathcal{G} \otimes \Omega_{X}^{1}$, (ii) $0<\operatorname{rank} \mathcal{G}<\operatorname{rank} \mathcal{E}$ :

$$
\frac{\operatorname{deg}\left(\mathcal{P}_{*} \mathcal{G}\right)}{\operatorname{rank} \mathcal{G}}<\frac{\operatorname{deg}\left(\mathcal{P}_{*} \mathcal{E}\right)}{\operatorname{rank} \mathcal{E}} \quad\left(\operatorname{resp} . \frac{\operatorname{deg}\left(\mathcal{P}_{*} \mathcal{G}\right)}{\operatorname{rank} \mathcal{G}} \leq \frac{\operatorname{deg}\left(\mathcal{P}_{*} \mathcal{E}\right)}{\operatorname{rank} \mathcal{E}}\right)
$$

The unramifiedly good filtered Higgs bundle $\left(\mathcal{P}_{*} \mathcal{E}, \theta\right)$ on $(X, D)$ is called polystable if it is the direct sum of stable ones $\left(\mathcal{P}_{*} \mathcal{E}_{i}, \theta_{i}\right)(i=1, \ldots, m)$ with $\operatorname{deg}\left(\mathcal{P}_{*} \mathcal{E}_{i}\right)=\operatorname{deg}\left(\mathcal{P}_{*} \mathcal{E}\right)$. In the following proposition, the tame case was proved by Simpson [20], and see [16] for the wild case, for example.

Proposition 3.3 Let $\left(E, \bar{\partial}_{E}, \theta, h\right)$ be an unramifiedly good wild harmonic bundle on $(X, D)$. Then, the associated filtered bundle $\left(\mathcal{P}_{*}^{h} E, \theta\right)$ on $(X, D)$ is poly-stable with $\operatorname{deg}\left(\mathcal{P}_{*}^{h} E\right)=0$.

In the following proposition, the tame case was proved by Simpson [20], and the wild case was proved by Biquard and Boalch [2].

Proposition 3.4 Let $\left(\mathcal{P}_{*} \mathcal{E}, \theta\right)$ be an unramifiedly good filtered Higgs bundle on $(X, D)$ with $\operatorname{deg}\left(\mathcal{P}_{*} \mathcal{E}\right)=0$. If $\left(\mathcal{P}_{*} \mathcal{E}, \theta\right)$ is stable, we have a harmonic metric $h$ of $(E, \theta)=(\mathcal{E}, \theta)_{\mid X \backslash D}$ with an isomorphism $\left(\mathcal{P}_{*}^{h} E, \theta\right) \simeq\left(\mathcal{P}_{*} \mathcal{E}, \theta\right)$. Such a metric $h$ is unique up to the multiplication of positive constants.

\subsubsection{Filtered bundles of rank one on curves}

Let $X$ be any complex curve with a discrete subset $D$. Let $L$ be a line bundle on $X$. Suppose that a tuple of real numbers $\boldsymbol{b}=\left(b_{P} \mid P \in D\right)$ is attached. Then, we have the filtered bundle $\mathcal{P}_{*}^{b} L$ over the meromorphic bundle $L(* D)$ given as follows. For $\boldsymbol{a}=\left(a_{P} \mid P \in D\right) \in \mathbb{R}^{D}$, we have the integers $n\left(a_{P}\right)(P \in D)$ such that $a_{P}-1<n\left(a_{P}\right)+b_{P} \leq a_{P}$, and we set

$$
\mathcal{P}_{\boldsymbol{a}}^{\boldsymbol{b}} L:=L\left(\sum_{P \in D} n\left(a_{P}\right) P\right) .
$$

If $X$ is compact, we have $\operatorname{deg}\left(\mathcal{P}_{*}^{b} L\right)=\operatorname{deg}(L)-\sum_{P \in D} b_{P}$.

\subsubsection{Comparison of filtered bundles}

Let $X$ be a compact connected Riemann surface with a finite subset $D$. Let $\mathcal{P}_{*} \mathcal{E}$ be a filtered bundle over a locally free $\mathcal{O}_{X}(* D)$-module $\mathcal{E}$ on $(X, D)$. Fix a point $P \in D$. Set $D_{1}:=D \backslash P$. For any $b \in \mathbb{R}$, let $\mathcal{P}_{b}^{P} \mathcal{E}$ denote the $\mathcal{O}_{X}\left(* D_{1}\right)$-module such that $\mathcal{P}_{b}^{P} \mathcal{E}(* P)=\mathcal{E}$ and the stalk of $\mathcal{P}_{b}^{P} \mathcal{E}$ at $P$ is $\mathcal{P}_{b}^{P}\left(\mathcal{E}_{P}\right)$. For any $b \in \mathbb{R}$ and $\boldsymbol{c} \in \mathbb{R}^{D_{1}}$, we set $\mathcal{P}_{\boldsymbol{c}}\left(\mathcal{P}_{b}^{P} \mathcal{E}\right):=\mathcal{P}_{(b, c)} \mathcal{E}$. For any $b \in \mathbb{R}$, we have the filtered bundle $\mathcal{P}_{*}\left(\mathcal{P}_{b}^{P} \mathcal{E}\right)=\left(\mathcal{P}_{\boldsymbol{c}} \mathcal{P}_{b}^{P} \mathcal{E} \mid \boldsymbol{c} \in \mathbb{R}^{D_{1}}\right)$ over $\mathcal{P}_{b}^{P} \mathcal{E}$ on $\left(X, D_{1}\right)$.

We have the set $\left\{a \mid 0<a \leq 1, \mathcal{P}_{a}^{P}\left(\mathcal{E}_{P}\right) / \mathcal{P}_{<a}^{P}\left(\mathcal{E}_{P}\right) \neq 0\right\}=\left\{a_{1}, \ldots, a_{\ell}\right\}$. We assume $a_{i}<a_{i+1}$. The following observation is given in [9]. 
Lemma 3.5 We have the following equality:

$$
\int_{0}^{1} \operatorname{deg}\left(\mathcal{P}_{*}\left(\mathcal{P}_{b}^{P} \mathcal{E}\right)\right) d b=\operatorname{deg}\left(\mathcal{P}_{*}\left(\mathcal{P}_{0}^{P} \mathcal{E}\right)\right)-\sum_{i=1}^{\ell}\left(a_{i}-1\right) \operatorname{dim}\left(\mathcal{P}_{a_{i}}^{P}\left(\mathcal{E}_{P}\right) / \mathcal{P}_{<a_{i}}^{P}\left(\mathcal{E}_{P}\right)\right)
$$

Here, we regard $\operatorname{deg}\left(\mathcal{P}_{*}\left(\mathcal{P}_{b}^{P} \mathcal{E}\right)\right)$ as a measurable function in variable b. In particular, we have $\operatorname{deg}\left(\mathcal{P}_{*} \mathcal{E}\right)=$ $\int_{0}^{1} \operatorname{deg}\left(\mathcal{P}_{*}\left(\mathcal{P}_{b}^{P} \mathcal{E}\right)\right) d b$.

Proof We obtain (22) by a direct computation. Because $\mathcal{P}_{a_{i}}^{P}\left(\mathcal{E}_{P}\right) / \mathcal{P}_{<a_{i}}^{P}\left(\mathcal{E}_{P}\right) \simeq \mathcal{P}_{a_{i}-1}^{P}\left(\mathcal{E}_{P}\right) / \mathcal{P}_{<a_{i}-1}^{P}\left(\mathcal{E}_{P}\right)$, we can observe the right hand side of $(22)$ is equal to $\operatorname{deg}\left(\mathcal{P}_{*} \mathcal{E}\right)$.

We recall a lemma for the comparison of filtered bundles for the convenience of the readers.

Lemma 3.6 Let $\mathcal{E}$ be a locally free $\mathcal{O}_{X}(* D)$-module. Let $\mathcal{P}_{*}^{i} \mathcal{E}(i=1,2)$ be filtered bundles over $\mathcal{E}$ such that $\mathcal{P}_{\boldsymbol{a}}^{1} \mathcal{E} \subset \mathcal{P}_{\boldsymbol{a}}^{2} \mathcal{E}$ for any $\boldsymbol{a} \in \mathbb{R}^{D}$. If $\operatorname{deg}\left(\mathcal{P}_{*}^{1} \mathcal{E}\right)=\operatorname{deg}\left(\mathcal{P}_{*}^{2} \mathcal{E}\right)$, then we have $\mathcal{P}_{\boldsymbol{a}}^{1} \mathcal{E}=\mathcal{P}_{\boldsymbol{a}}^{2} \mathcal{E}$ for any $\boldsymbol{a} \in \mathbb{R}^{D}$.

Proof We use an induction on $|D|$. If $D=\emptyset$, the claim is clear. Take $P \in D$, and set $D_{1}:=D \backslash\{P\}$. We obtain the filtered bundles $\mathcal{P}_{*}^{i} \mathcal{P}_{b}^{P} \mathcal{E}(i=1,2)$ for any $b \in \mathbb{R}$ as above. By using Lemma 3.5 , we can easily deduce $\operatorname{deg}\left(\mathcal{P}_{*}^{1}\left(\mathcal{P}_{b}^{P} \mathcal{E}\right)\right)=\operatorname{deg}\left(\mathcal{P}_{*}^{2}\left(\mathcal{P}_{b}^{P} \mathcal{E}\right)\right)$ for any $b \in \mathbb{R}$ from the equality $\operatorname{deg}\left(\mathcal{P}_{*}^{1} \mathcal{E}\right)=\operatorname{deg}\left(\mathcal{P}_{*}^{2} \mathcal{E}\right)$. By the hypothesis of the induction, we obtain $\mathcal{P}_{\boldsymbol{c}}^{1}\left(\mathcal{P}_{b}^{P} \mathcal{E}\right)=\mathcal{P}_{c}^{2}\left(\mathcal{P}_{b}^{P} \mathcal{E}\right)$ for any $\boldsymbol{c}$. Then, the claim of the lemma follows.

\subsection{Filtered Higgs bundles of rank 2 on $\left(\mathbb{P}^{1}, \infty\right)$}

Let $\zeta$ be the standard coordinate on $\mathbb{C} \subset \mathbb{P}^{1}$. We set $\widetilde{V}=\mathcal{O}_{\mathbb{P}^{1}}(* \infty) \widetilde{v}_{1} \oplus \mathcal{O}_{\mathbb{P}^{1}}(* \infty) \widetilde{v}_{2}$. Take a non-zero complex number $\alpha$ and a positive integer $m$. We have the Higgs field $\widetilde{\theta}$ on $\widetilde{V}$ given by

$$
\widetilde{\theta}\left(\widetilde{v}_{1}, \widetilde{v}_{2}\right)=\left(\widetilde{v}_{1}, \widetilde{v}_{2}\right)\left(\begin{array}{cc}
\alpha \zeta^{m} d \zeta & 0 \\
0 & -\alpha \zeta^{m} d \zeta
\end{array}\right)
$$

Fix $0 \leq \ell \leq m$. Let $\widetilde{E}_{\ell} \subset \widetilde{V}$ be the $\mathcal{O}_{\mathbb{P}^{1}}(* \infty)$-submodule generated by $\widetilde{e}_{1}=\widetilde{v}_{1}+\widetilde{v}_{2}$ and $\widetilde{e}_{2}=\zeta^{\ell} \widetilde{v}_{2}$. Because $\widetilde{\theta}\left(\widetilde{E}_{\ell}\right) \subset \widetilde{E}_{\ell} \otimes \Omega_{\mathbb{P}^{1}}^{1}$, we obtain the meromorphic Higgs bundle $\left(\widetilde{E}_{\ell}, \widetilde{\theta}\right)$. We have

$$
\widetilde{\theta}\left(\widetilde{e}_{1}, \widetilde{e}_{2}\right)=\left(\widetilde{e}_{1}, \widetilde{e}_{2}\right)\left(\begin{array}{cc}
\alpha \zeta^{m} d \zeta & 0 \\
-2 \alpha \zeta^{m-\ell} d \zeta & -\alpha \zeta^{m} d \zeta
\end{array}\right)
$$

Take $c \in \mathbb{R}$, and set $c_{1}:=c$ and $c_{2}:=-c-\ell$. We have the parabolic Higgs bundle $\left(\mathcal{P}_{*}^{c} \widetilde{E}_{\ell}, \widetilde{\theta}\right)$ on $\left(\mathbb{P}^{1}, \infty\right)$ with $\operatorname{deg}\left(\mathcal{P}_{*}^{c} \widetilde{E}_{\ell}\right)=0$ given as follows

$$
\mathcal{P}_{b}^{c}\left(\widetilde{E}_{\ell}\right)_{\mid \mathbb{P}^{1} \backslash\{\infty\}}=\left(\mathcal{O}_{\mathbb{P}^{1}}\left(\left[b-c_{1}\right] \infty\right) \widetilde{v}_{1} \oplus \mathcal{O}_{\mathbb{P}^{1}}\left(\left[b-c_{2}\right] \infty\right) \widetilde{v}_{2}\right)_{\mid \mathbb{P}^{1} \backslash\{\infty\}}
$$

Here, $[x]:=\max \{n \in \mathbb{Z} \mid n \leq x\}$. We shall impose that $c_{1} \geq c_{2}$, i.e., $c \geq-\ell / 2$.

\subsubsection{Stability condition}

We set $L_{i}:=\mathcal{O}_{\mathbb{P}^{1}}(* \infty) \widetilde{v}_{i} \subset \widetilde{V}(i=1,2)$. We have $\widetilde{E}_{\ell} \cap L_{i}=\mathcal{O}_{\mathbb{P}^{1}}(* \infty) \cdot \zeta^{\ell} \widetilde{v}_{i}$. We have the induced parabolic bundle $\mathcal{P}_{*}^{c}\left(\widetilde{E}_{\ell} \cap L_{i}\right)$. We have $\operatorname{deg}\left(\mathcal{P}_{*}^{c}\left(\widetilde{E}_{\ell} \cap L_{i}\right)\right)=-\ell-c_{i}$. Hence, $\left(\mathcal{P}_{*}^{c} \widetilde{E}_{\ell}, \widetilde{\theta}\right)$ is stable if and only if $-\ell-c_{i}<0$ $(i=1,2)$, i.e., $c_{i}<0(i=1,2)$. We also have that $\left(\mathcal{P}_{*}^{c} \widetilde{E}_{\ell}, \widetilde{\theta}\right)$ is semistable if and only if $-\ell-c_{1}=0$ or $-\ell-c_{2}=0$, i.e., $c_{1}=0,-\ell$. Under the assumption $c_{1} \geq c_{2}$, the semistability is equivalent to $c_{1}=0$.

\subsubsection{The determinant bundles}

We have $\operatorname{det}\left(\widetilde{E}_{\ell}\right)=\mathcal{O}_{\mathbb{P}^{1}}(* \infty) \cdot \widetilde{e}_{1} \wedge \widetilde{e}_{2}=\mathcal{O}_{\mathbb{P}^{1}}(* \infty) \cdot \zeta^{\ell} \widetilde{v}_{1} \wedge \widetilde{v}_{2}$. The induced filtered bundle $\operatorname{det}\left(\mathcal{P}_{*}^{c} \widetilde{E}_{\ell}, \widetilde{\theta}\right)$ over $\operatorname{det}\left(\widetilde{E}_{\ell}\right)$ is equal to $\mathcal{P}_{*}^{0}\left(\mathcal{O}_{\mathbb{P}} \cdot \widetilde{e}_{1} \wedge \widetilde{e}_{2}\right)$, where $\mathcal{P}_{b}^{0}\left(\mathcal{O}_{\mathbb{P}^{1}} \cdot \widetilde{e}_{1} \wedge \widetilde{e}_{2}\right)=\mathcal{O}_{\mathbb{P}^{1}}([b]) \cdot \widetilde{e}_{1} \wedge \widetilde{e}_{2}$ for $[b]:=\max \{n \in \mathbb{Z} \mid n \leq b\}$. In particular, they are independent of $c$. We fix the Hermitian metric $h_{\operatorname{det}\left(\widetilde{E}_{\ell}\right)}$ of $\operatorname{det}\left(\widetilde{E}_{\ell}\right) \operatorname{given} \operatorname{by} h_{\operatorname{det}\left(\widetilde{E}_{\ell}\right)}\left(\widetilde{e}_{1} \wedge \widetilde{e}_{2}, \widetilde{e}_{1} \wedge \widetilde{e}_{2}\right)=1$. 


\subsubsection{Harmonic metrics in the case $\ell=0$}

If $\ell=0,\left(\mathcal{P}_{*}^{c} \widetilde{E}_{0}, \widetilde{\theta}\right)$ cannot be stable. And, $\left(\mathcal{P}_{*}^{c} \widetilde{E}_{0}, \widetilde{\theta}\right)$ is semistable if and only if $c=0$. Indeed, it is polystable in that case, i.e., we have the decomposition $\left(\mathcal{P}_{*}^{0} \widetilde{E}_{0}, \widetilde{\theta}\right)=\left(\mathcal{P}_{*}^{0} L_{1}, \widetilde{\theta}_{1}\right) \oplus\left(\mathcal{P}_{*}^{0} L_{2}, \widetilde{\theta}_{2}\right)$, where $\widetilde{\theta}_{1}$ and $\widetilde{\theta}_{2}$ are the multiplications of $\alpha \zeta^{m} d \zeta$ and $-\alpha \zeta^{m} d \zeta$, respectively. We have the harmonic metrics $h_{L_{i}}$ for $\left(\mathcal{P}_{*}^{0} L_{i}, \widetilde{\theta}_{i}\right)$. We impose $h_{L_{1}} \otimes h_{L_{2}}=h_{\operatorname{det}\left(\widetilde{E}_{0}\right)}$. Then, we have a harmonic metric $h_{\widetilde{E}_{0}}=h_{L_{1}} \oplus h_{L_{2}}$ for $\left(\mathcal{P}_{*}^{0} \widetilde{E}_{0}, \widetilde{\theta}\right)$. Note that we have an ambiguity given by automorphisms of $\left(\mathcal{P}_{*}^{0} \widetilde{E}_{0}, \widetilde{\theta}\right)$, i.e., for any $\alpha>0, \alpha h_{L_{1}} \oplus \alpha^{-1} h_{L_{2}}$ is also a harmonic metric for $\left(\mathcal{P}_{*}^{0} \widetilde{E}_{0}, \widetilde{\theta}\right)$. We also note that $h_{L_{i}}\left(\widetilde{v}_{i}, \widetilde{v}_{i}\right)$ are constants.

\subsubsection{Harmonic metrics in the case $\ell>0$ and their homogeneous property}

Suppose that $\ell>0$. According to Proposition 3.4, if the unramifiedly good filtered Higgs bundle $\left(\mathcal{P}_{*}^{c} \widetilde{E}_{\ell}, \widetilde{\theta}\right)$ is stable, i.e., $-\ell<c<0$, we have the harmonic metric $h_{c, \ell}$ of $\left(\widetilde{E}_{\ell}, \widetilde{\theta}\right)_{\mid \mathbb{C}}$ adapted to the filtered Higgs bundle $\left(\mathcal{P}_{*}^{c} \widetilde{E}_{\ell}, \widetilde{\theta}\right) \operatorname{such}$ that $\operatorname{det}\left(h_{c, \ell}\right)=h_{\operatorname{det} \widetilde{E}_{\ell}}$.

For any $\tau \in \mathbb{C}^{*}$, let $\varphi_{\tau}: \mathbb{P}^{1} \longrightarrow \mathbb{P}^{1}$ be given by $\varphi_{\tau}(\zeta)=\tau^{2} \zeta$. We have the isomorphism $\Phi_{\tau}: \varphi_{\tau}^{*} \widetilde{E}_{\ell} \simeq \widetilde{E}_{\ell}$ given by $\tau^{\ell} \varphi_{\tau}^{*} \widetilde{e}_{1} \longleftrightarrow \widetilde{e}_{1}$ and $\tau^{-\ell} \varphi_{\tau}^{*} \widetilde{e}_{2} \longleftrightarrow \widetilde{e}_{2}$. Under the isomorphism, we have $\tau^{\ell} \varphi_{\tau}^{*} \widetilde{v}_{i} \longleftrightarrow \widetilde{v}_{i}(i=1,2)$. Hence, $\Phi_{\tau}$ induces an isomorphism of the filtered bundles $\varphi_{\tau}^{*} \mathcal{P}_{*} \widetilde{E}_{\ell} \simeq \mathcal{P}_{*} \widetilde{E}_{\ell}$. Under the isomorphism, we have $\varphi_{\tau}^{*} \widetilde{\theta}=\tau^{2(m+1)} \widetilde{\theta}$. Hence, the Hermitian metric $\varphi_{\tau}^{*} h_{c, \ell}$ gives a harmonic metric of $\left(\mathcal{P}_{*}^{c} \widetilde{E}_{\ell}, \tau^{2(m+1)} \widetilde{\theta}\right)$. Note that $\operatorname{det} \varphi^{*} h_{c, \ell}=h_{\operatorname{det} \widetilde{E}_{\ell}}$.

Proposition 3.7 If $|\tau|=1$, we have $\varphi_{\tau}^{*} h_{c, \ell}=h_{c, \ell}$.

Proof If $|\tau|=1$, both the Hermitian metrics $\varphi_{\tau}^{*} h_{c, \ell}$ and $h_{c, \ell}$ are harmonic metrics of $\left(\widetilde{E}_{\ell}, \widetilde{\theta}\right)$. They are adapted to the filtered bundle $\mathcal{P}_{*}^{c} \widetilde{E}_{\ell}$, and they satisfy $\operatorname{det}\left(h_{c, \ell}\right)=\operatorname{det}\left(\varphi_{\tau}^{*} h_{c, \ell}\right)=h_{\operatorname{det} \widetilde{E}_{\ell}}$. Hence, we have $h_{c, \ell}=\varphi_{\tau}^{*} h_{c, \ell}$ by the uniqueness.

Corollary 3.8 The functions $h_{c, \ell}\left(\widetilde{e}_{i}, \widetilde{e}_{i}\right)(i=1,2)$ depend only on $|\zeta|$. The function $\zeta^{-\ell} h_{c, \ell}\left(\widetilde{e}_{1}, \widetilde{e}_{2}\right)$ depend only on $|\zeta|$. The functions $h_{c, \ell}\left(\widetilde{v}_{i}, \widetilde{v}_{j}\right)(i, j \in\{1,2\})$ depend only on $|\zeta|$.

Remark 3.9 We clearly have the homogeneous property of the harmonic metrics such as Corollary 3.8 even in the case $\ell=0$.

\subsubsection{The norms of $\widetilde{v}_{j}$}

By the norm estimate of wild harmonic bundles [16], we have constants $C_{i, c}>0(i=1,2)$ depending on $c$ such that $C_{1, c}|\zeta|^{c_{j}} \leq\left|\widetilde{v}_{j}\right|_{h_{c, \ell}} \leq C_{2, c}|\zeta|^{c_{j}}$, where $c_{1}=c$ and $c_{2}=-c-\ell$. Let us refine it in our situation.

Proposition 3.10 There exist positive constants $b_{c}, C_{3}$ and $\epsilon_{3}$ such that the following holds on $\{|\zeta|>1\}$ :

$$
\begin{gathered}
\left.|\log | \widetilde{v}_{1}\right|_{h_{c, \ell}}-\log \left(b_{c}|\zeta|^{c_{1}}\right) \mid \leq C_{3} \exp \left(-\epsilon_{3}|\zeta|^{m+1}\right) \\
|\log | \widetilde{v}_{2}||_{h_{c, \ell}}-\log \left(b_{c}^{-1}|\zeta|^{c_{2}}\right) \mid \leq C_{3} \exp \left(-\epsilon_{3}|\zeta|^{m+1}\right) \\
\left.\left|\zeta \partial_{\zeta} \log \right| \widetilde{v}_{i}\right|_{h_{c, \ell}}-c_{i} / 2 \mid \leq C_{3} \exp \left(-\epsilon_{3}|\zeta|^{m+1}\right) \quad(i=1,2)
\end{gathered}
$$

Here, $b_{c}$ may depend on $c$, but $C_{3}$ and $\epsilon_{3}$ are independent of $c$.

Proof Let $\eta:=\zeta^{-1}$ be the coordinate around $\infty$. Set $U_{\infty}=\{|\eta| \leq 1\} \subset \mathbb{P}^{1}$.

Lemma 3.11 There exist $C_{4}>0$ and $\epsilon_{4}>0$ which are independent of $c$, such that the following holds on $U_{\infty} \backslash\{\infty\}:$

$$
\left|\partial_{\eta} \partial_{\bar{\eta}}\left(\log \left|\widetilde{v}_{j}\right|_{h_{c, \ell}}\right)\right| \leq C_{4} \exp \left(-\epsilon_{4}|\eta|^{-m-1}\right)
$$


Proof Let $h_{L_{j}, c, \ell}$ denote the restriction of $h_{c, \ell}$ to $L_{j}$. Let $R\left(h_{L_{j}, c, \ell}\right)$ denote the curvature of the Chern connection of $\left(L_{j}, h_{L_{j}, c, \ell}\right)$. Let $g_{\mathbb{C}}$ be the Euclidean metric $d \zeta d \bar{\zeta}$. By Proposition 2.12, we have positive constants $C_{5}$ and $\epsilon_{5}$ which are independent of $c$, such that the following holds:

$$
\left|R\left(h_{L_{j}, c, \ell}\right)\right|_{h_{L_{j}, c, \ell}, g_{\mathrm{C}}} \leq C_{5} \exp \left(-\epsilon_{5}|\zeta|^{m+1}\right)
$$

Because $\bar{\partial} \partial \log \left|\widetilde{v}_{j}\right|_{h_{c, \ell}}=R\left(h_{L_{j}, c, \ell}\right)$, we obtain the claim of the lemma.

We set $Y_{\widetilde{\eta}}:=\{\widetilde{\eta} \in \mathbb{C}|| \widetilde{\eta} \mid<1\}$. For any $\kappa<1$, we have the isomorphism $\Psi_{\kappa}: Y_{\widetilde{\eta}} \longrightarrow\{|\eta|<\kappa\}$ given by $\Psi_{\kappa}(\widetilde{\eta})=\kappa \widetilde{\eta}$. We have the following on $Y_{\widetilde{\eta}} \backslash\{0\}$ :

$$
\left|\partial_{\widetilde{\eta}} \partial_{\widetilde{\eta}} \Psi_{\kappa}^{*}\left(\log \left|\widetilde{v}_{1}\right|_{h_{c, \ell}}\right)\right| \leq C_{4} \kappa^{2} \exp \left(-\epsilon_{4} \kappa^{-m-1}|\widetilde{\eta}|^{-m-1}\right)
$$

Take a large $p>1$. For each $c$ and $\kappa$, we can take an $\mathbb{R}$-valued $L_{2}^{p}$-function $G_{\kappa, c}$ on a neighbourhood of the closure of $Y_{\widetilde{\eta}}$ such that (i) $G_{\kappa, c}$ is a function of $|\eta|$, (ii) there exist positive constants $C_{6}$ and $\epsilon_{6}$ such that the $L_{2}^{p}$-norm of $G_{\kappa, c}$ on $Y_{\widetilde{\eta}}$ is dominated by $C_{6} \exp \left(-\epsilon_{6} \kappa^{-m-1}\right)$, (iii) $G_{\kappa, c}(0)=0$, (iv) the following holds on $Y_{\widetilde{\eta}}$ :

$$
\partial_{\widetilde{\eta}} \partial_{\widetilde{\eta}}\left(\Psi_{\kappa}^{*}\left(\log \left|\widetilde{v}_{1}\right|_{h_{c, \ell}}\right)-G_{\kappa, c}\right)=0
$$

Because $\Psi_{\kappa}^{*}\left(\log \left|\widetilde{v}_{1}\right|_{h_{c, \ell}}\right)-G_{\kappa, c}-\log |\widetilde{\eta}|^{-c_{1}}$ is bounded, we have a holomorphic function $g_{\kappa, c}$ on $Y_{\widetilde{\eta}}$ such that $\Psi_{\kappa}^{*}\left(\log \left|\widetilde{v}_{1}\right|_{h_{c, \ell}}\right)-G_{\kappa, c}-\log |\widetilde{\eta}|^{-c_{1}}=\operatorname{Re}\left(g_{\kappa, c}\right)$. Because $\Psi_{\kappa}^{*}\left(\log \left|\widetilde{v}_{1}\right|_{h_{c, \ell}}\right)-G_{\kappa, c}-\log |\widetilde{\eta}|^{-c_{1}}$ depends only on $|\eta|$, we obtain that $g_{\kappa, c}$ is constant. We also obtain

$$
\widetilde{\eta} \partial_{\widetilde{\eta}} \Psi_{\kappa}^{*}\left(\log \left|\widetilde{v}_{1}\right|_{h_{c, \ell}}\right)+c_{1} / 2-\widetilde{\eta}_{\partial_{\tilde{\eta}}} G_{\kappa, c}=0 .
$$

Hence, on $\{|\eta|<\kappa\}$, the function $\log \left|\widetilde{v}_{1}\right|_{h_{c, \ell}}-\left(\Psi_{\kappa}^{-1}\right)^{*} G_{\kappa, c}-\log |\eta|^{-c_{1}}$ is a constant. We have positive constants $C_{7}$ and $\epsilon_{7}$ such that the following holds on $\{\kappa / 2<|\eta|<\kappa\}$ :

$$
\begin{gathered}
\left|\left(\Psi_{\kappa}^{-1}\right)^{*} G_{\kappa, c}\right| \leq C_{7} \exp \left(-\epsilon_{7}|\eta|^{-m-1}\right) \\
\left|\eta \partial_{\eta}\left(\Psi_{\kappa}^{-1}\right)^{*} G_{\kappa, c}\right| \leq C_{7} \exp \left(-\epsilon_{7}|\eta|^{-m-1}\right)
\end{gathered}
$$

We also obtain that the function $F:=\log \left|\widetilde{v}_{1}\right|_{h_{c, \ell}}-\log |\eta|^{-c_{1}}$ gives a continuous function on $\{|\eta|<1\}$. We set $b_{c}:=\exp (F(0))$. Then, we obtain (23), and (25) for $i=1$. By using $\left|\widetilde{v}_{1} \wedge \widetilde{v}_{2}\right|_{h_{c, \ell}}=|\zeta|^{-\ell}$ and the estimate (26) below, we obtain the estimate (24). We also obtain (25) for $i=2$ with a similar argument.

\subsubsection{Asymptotic orthogonality and some complements}

As studied in [16], we have the following estimate on $\{|\zeta| \geq 1\}$, which also follows from Corollary 2.6:

$$
\left|h_{c, \ell}\left(\widetilde{v}_{1}, \widetilde{v}_{2}\right)\right| \leq K \exp \left(-\delta|\zeta|^{m+1}\right) \cdot\left|\widetilde{v}_{1}\right|_{h_{c, \ell}} \cdot\left|\widetilde{v}_{2}\right|_{h_{c, \ell}} \leq K^{\prime} \exp \left(-\delta|\zeta|^{m+1}\right)
$$

Here, $K, K^{\prime}$ and $\delta$ are positive constants which are independent of $c$.

Let $\bar{\partial}_{c, \ell}$ denote the holomorphic structure of $\widetilde{E}_{\ell}$. Let $\partial_{c, \ell}$ denote the $(1,0)$-part of the Chern connection of $\left(\widetilde{E}_{\ell}, h_{c, \ell}\right)$. Let $g_{\mathbb{C}}$ denote the Euclidean metric of $\mathbb{C}_{\zeta}$.

Lemma 3.12 We have positive constants $K_{1}$ which are independent of $c$, such that $\left|\zeta \partial_{c, \ell} \widetilde{v}_{i}\right|_{h_{c, \ell}, g_{\mathbb{C}}} \leq K_{1} \cdot\left|\widetilde{v}_{i}\right|_{h_{c, \ell}}$ on $\{|\zeta| \geq 1\}$.

Proof In this proof, the constants $K_{i}$ and $\delta_{i}$ are independent of $c$. To simplify the description, we denote $h_{c, \ell}$ by $h$. By the asymptotic orthogonality (26), we have a positive constant $K_{2}$ such that the following holds on $\{|\zeta| \geq 1\}$ :

$$
\left|\partial_{c, \ell} \widetilde{v}_{1}\right|_{h, g_{\mathbb{C}}} \leq K_{2}\left(\left|\widetilde{v}_{1}\right|_{h}^{-1} \cdot\left|h\left(\partial_{c, \ell} \widetilde{v}_{1}, \widetilde{v}_{1}\right)\right|_{g_{\mathbb{C}}}+\left|\widetilde{v}_{2}\right|_{h}^{-1} \cdot\left|h\left(\partial_{c, \ell} \widetilde{v}_{1}, \widetilde{v}_{2}\right)\right|_{g_{\mathbb{C}}}\right)
$$


By Proposition 3.10, we have a constant $K_{3}>0$ such that the following holds on $\{|\zeta| \geq 1\}$ :

$$
\left|\widetilde{v}_{1}\right|_{h}^{-1}\left|h\left(\partial_{c, \ell} \widetilde{v}_{1}, \widetilde{v}_{1}\right)\right|_{g_{\mathbb{C}}}=\left.\left.\left|\widetilde{v}_{1}\right|_{h} \cdot|\partial \log | \widetilde{v}_{1}\right|_{h} ^{2}\right|_{g_{\mathbb{C}}} \leq K_{3}\left|\widetilde{v}_{1}\right|_{h} \cdot|\zeta|^{-1}
$$

Let $\pi_{1}$ denote the projection of $\widetilde{V}=L_{1} \oplus L_{2}$ onto $L_{1}$. We also have the following on $\{|\zeta| \geq 1\}$ :

$$
\left|\widetilde{v}_{2}\right|_{h}^{-1} \cdot\left|h\left(\partial_{c, \ell} \widetilde{v}_{1}, \widetilde{v}_{2}\right)\right|_{g_{\mathbb{C}}} \leq\left|\widetilde{v}_{2}\right|_{h}^{-1} \cdot\left|h\left(\left(\partial_{c, \ell} \pi_{1}\right) \cdot \widetilde{v}_{1}, \widetilde{v}_{2}\right)\right|+\left|\widetilde{v}_{2}\right|_{h}^{-1} \cdot\left|h\left(\pi_{1}\left(\partial_{c, \ell} \widetilde{v}_{1}\right), \widetilde{v}_{2}\right)\right|
$$

By Proposition 2.10, we have positive constants $K_{4}$ and $\delta_{4}$ such that

$$
\left|\partial_{c, \ell} \pi_{1}\right|_{h, g_{\mathbb{C}}} \leq K_{4} \exp \left(-\delta_{4}|\zeta|^{m+1}\right)
$$

By (26) and Proposition 2.3, we have positive constants $K_{5}$ and $\delta_{5}$ such that

$$
\left|h\left(\pi_{1}\left(\partial_{c, \ell} \widetilde{v}_{1}\right), \widetilde{v}_{2}\right)\right| \leq K_{5} \exp \left(-\delta_{5}|\zeta|^{m+1}\right)\left|\partial_{c, \ell} \widetilde{v}_{1}\right|_{h, g_{\mathbb{C}}} \cdot\left|\widetilde{v}_{2}\right|_{h}
$$

Hence, we obtain the following on $\{|\zeta| \geq 1\}$ :

$$
\left|\partial_{c, \ell} \widetilde{v}_{1}\right|_{h, g_{\mathbb{C}}} \leq K_{2}\left(K_{3}|\zeta|^{-1}+K_{4} \exp \left(-\delta_{4}|\zeta|^{m+1}\right)\right)\left|\widetilde{v}_{1}\right|_{h}+K_{2} K_{5} \exp \left(-\delta_{5}|\zeta|^{m+1}\right)\left|\partial_{c, \ell} \widetilde{v}_{1}\right|_{h, g_{\mathbb{C}}}
$$

Hence, we obtain the desired estimate for $\partial_{c, \ell} \widetilde{v}_{1}$. Similarly, we obtain the estimate for $\partial_{c, \ell} \widetilde{v}_{2}$.

Lemma 3.13 We have positive constants $K_{6}$ and $\delta_{6}$ which are independent of $c$, such that the following holds:

$$
\begin{gathered}
\left|\partial_{\zeta} h_{c, \ell}\left(\widetilde{v}_{1}, \widetilde{v}_{2}\right)\right| \leq K_{6} \exp \left(-\delta_{6}|\zeta|^{m+1}\right), \quad\left|\partial_{\bar{\zeta}} h_{c, \ell}\left(\widetilde{v}_{1}, \widetilde{v}_{2}\right)\right| \leq K_{6} \exp \left(-\delta_{6}|\zeta|^{m+1}\right), \\
\left|\partial_{\zeta} \partial_{\bar{\zeta}} h_{c, \ell}\left(\widetilde{v}_{1}, \widetilde{v}_{2}\right)\right| \leq K_{6} \exp \left(-\delta_{6}|\zeta|^{m+1}\right) .
\end{gathered}
$$

Proof In this proof, the constants $K_{i}$ and $\delta_{i}$ are independent of $c$. We obtain the estimate for $\partial_{\zeta} h_{c, \ell}\left(\widetilde{v}_{1}, \widetilde{v}_{2}\right)$ from (27-29) and Lemma 3.12. We obtain the estimate for $\partial_{\bar{\zeta}} h_{c, \ell}\left(\widetilde{v}_{1}, \widetilde{v}_{2}\right)$ in a similar way. Let $R\left(h_{c, \ell}\right)$ denote the curvature of $\left(\widetilde{E}_{\ell}, h_{c, \ell}\right)$. We have the following:

$$
\partial \bar{\partial} h_{c, \ell}\left(\widetilde{v}_{1}, \widetilde{v}_{2}\right)=h_{c, \ell}\left(\partial_{c, \ell} \widetilde{v}_{1}, \partial_{c, \ell} \widetilde{v}_{2}\right)+h_{c, \ell}\left(\widetilde{v}_{1}, R\left(h_{c, \ell}\right) \widetilde{v}_{2}\right)
$$

We have positive constants $K_{7}$ and $\delta_{7}$ such that $\left|R\left(h_{c, \ell}\right)\right|_{h_{c, \ell}, g_{\mathbb{C}}} \leq K_{7} \exp \left(-\delta_{n}|\zeta|^{m+1}\right)$. Hence, we have

$$
\left|h_{c, \ell}\left(\widetilde{v}_{1}, R\left(h_{c, \ell}\right) \widetilde{v}_{2}\right)\right|_{g_{\mathbb{C}}} \leq K_{8} \exp \left(-\delta_{8}|\zeta|^{m+1}\right)
$$

for positive constants $K_{8}$ and $\delta_{8}$. Let $\pi_{i}$ denote the projection of $\widetilde{V}=L_{1} \oplus L_{2}$ to $L_{i}$. We have the following:

$$
\begin{array}{r}
h_{c, \ell}\left(\partial_{c, \ell} \widetilde{v}_{1}, \partial_{c, \ell} \widetilde{v}_{2}\right)=h_{c, \ell}\left(\pi_{1}\left(\partial_{c, \ell} \widetilde{v}_{1}\right), \pi_{2}\left(\partial_{c, \ell} \widetilde{v}_{2}\right)\right)+h_{c, \ell}\left(\left(\partial_{c, \ell} \pi_{1}\right) \widetilde{v}_{1}, \pi_{2}\left(\partial_{c, \ell} \widetilde{v}_{2}\right)\right) \\
+h_{c, \ell}\left(\pi_{1}\left(\partial_{c, \ell} \widetilde{v}_{1}\right),\left(\partial_{c, \ell} \pi_{2}\right) \widetilde{v}_{2}\right)+h_{c, \ell}\left(\left(\partial_{c, \ell} \pi_{1}\right) \widetilde{v}_{1},\left(\partial_{c, \ell} \pi_{2}\right) \widetilde{v}_{2}\right)
\end{array}
$$

Hence, as in the case of the estimate for $h_{c, \ell}\left(\partial_{c, \ell} \widetilde{v}_{1}, \widetilde{v}_{2}\right)$, we have positive constants $K_{9}$ and $\delta_{9}$ such that $\left|h_{c, \ell}\left(\partial_{c, \ell} \widetilde{v}_{1}, \partial_{c, \ell} \widetilde{v}_{2}\right)\right|_{g_{\mathbb{C}}} \leq K_{9} \exp \left(-\delta_{9}|\zeta|^{m+1}\right)$. Thus, we obtain the desired estimate for $\partial_{\zeta} \partial_{\bar{\zeta}} h_{c, \ell}\left(\widetilde{v}_{1}, \widetilde{v}_{2}\right)$.

\subsubsection{Convergence of some sequences}

Set $t:=\tau^{2(m+1)}$ for $\tau \in \mathbb{C}^{*}$. We use the notation in $\S 3.2 .4$. We have the family of the harmonic metrics $h_{t, c, \ell}:=\varphi_{\tau}^{*} h_{c, \ell}$ for $\left(\mathcal{P}_{*}^{c} \widetilde{E}_{\ell}, t \widetilde{\theta}\right)$ satisfying $\operatorname{det}\left(h_{t, c, \ell}\right)=h_{\operatorname{det}(E)}$.

Under the isomorphism $\Phi_{\tau}: \varphi_{\tau}^{*} \widetilde{E}_{\ell} \simeq \widetilde{E}_{\ell}$, we have $\tau^{\ell} \varphi_{\tau}^{*} \widetilde{v}_{i} \longleftrightarrow \widetilde{v}_{i}(i=1,2)$. Take any $T>0$. We have positive constants $C$ and $\epsilon$, which are independent of $c$ and $T$, such that the following holds on $\{|\zeta| \geq T\}$ for any $t$ satisfying $|t|>T^{-1}$ :

$$
\left.|\log | \widetilde{v}_{1}\right|_{h_{t, c, \ell}}-\log \left(b_{c}|t|^{(\ell+2 c) / 2(m+1)}|\zeta|^{c}\right) \mid \leq C \exp \left(-\epsilon|\zeta|^{m+1}|t|\right)
$$




$$
\begin{gathered}
\left.|\log | \widetilde{v}_{2}\right|_{h_{t, c, \ell}}-\log \left(b_{c}^{-1}|t|^{-(\ell+2 c) / 2(m+1)}|\zeta|^{-c-\ell}\right) \mid \leq C \exp \left(-\epsilon|\zeta|^{m+1}|t|\right) \\
\left|h_{t, c, \ell}\left(\widetilde{v}_{1}, \widetilde{v}_{2}\right)\right| \leq C \exp \left(-\epsilon|\zeta|^{m+1}|t|\right) \cdot\left|\widetilde{v}_{1}\right|_{h_{t, c, \ell}} \cdot\left|\widetilde{v}_{2}\right|_{h_{t, c, \ell}} \\
\left|R\left(h_{t, c, \ell}\right)\right|_{h_{t, c, \ell}, g_{\mathrm{C}}} \leq C \exp \left(-\epsilon|\zeta|^{m+1}|t|\right)
\end{gathered}
$$

Here, $g_{\mathbb{C}}$ denote the standard Euclidean metric on $\mathbb{C}$.

Let $h_{c, \ell}^{\lim }$ be the Hermitian metric of $\widetilde{V}_{\mid \mathbb{C}^{*}}$ given by

$$
h_{c, \ell}^{\lim }\left(\widetilde{v}_{1}, \widetilde{v}_{1}\right)=|\zeta|^{2 c}, \quad h_{c, \ell}^{\lim }\left(\widetilde{v}_{2}, \widetilde{v}_{2}\right)=|\zeta|^{-2 c-2 \ell}, \quad h_{c, \ell}^{\lim }\left(\widetilde{v}_{1}, \widetilde{v}_{2}\right)=0 .
$$

For any $\gamma>0$, the automorphism $\Psi_{\gamma}$ on $\widetilde{V}_{\mid \mathbb{C}^{*}}$ is given by $\Psi_{\gamma}=\gamma \operatorname{id}_{L_{1}} \oplus \gamma^{-1} \operatorname{id}_{L_{2}}$. We define $\Psi_{\gamma}^{*} h_{t, c, \ell}\left(u_{1}, u_{2}\right):=$ $h_{t, c, \ell}\left(\Psi_{\gamma} u_{1}, \Psi_{\gamma} u_{2}\right)$. The following is clear.

Proposition 3.14 Set $\gamma(t):=b_{c}^{-1} t^{-(\ell+2 c) / 2(m+1)}$. Then, we have the convergence $\lim _{|t| \rightarrow \infty} \Psi_{\gamma(t)}^{*} h_{t, c, \ell}=h_{c, \ell} \lim$ on $\mathbb{C}^{*}$. For any fixed $T>0$, we have a constant $\delta_{T}>0$ depending on $T$, such that the order of the convergence is $\exp \left(-\delta_{T}|t|\right)$ on $\{|\zeta| \geq T\}$.

\subsection{Family of harmonic metrics in the case $\ell>0$}

We continue to use the notation in $\S 3.2$. We study the dependence of the harmonic metrics $h_{c, \ell}$ on $c$ in the case $\ell>0$.

\subsubsection{Continuity of $b_{c}$ with respect to the parabolic weights}

Proposition $3.15 b_{c}$ is continuous with respect to $c$.

Proof Fix $-\ell<c^{0}<0$. It is enough to study the continuity at $c^{0}$. We give a preliminary. Take a neighbourhood $U$ of $c^{0}$. We have a family of Hermitian metrics $h_{c, \ell}^{0}(c \in U)$ of $\widetilde{E}_{\ell}$ satisfying the following conditions:

- We have $h_{c^{0}, \ell}^{0}=h_{c^{0}, \ell}$ on $\mathbb{C}$.

- We have $h_{c, \ell}^{0}\left(\widetilde{v}_{1}, \widetilde{v}_{2}\right)=h_{c^{0}, \ell}\left(\widetilde{v}_{1}, \widetilde{v}_{2}\right), h_{c, \ell}^{0}\left(\widetilde{v}_{1}, \widetilde{v}_{1}\right)=h_{c^{0}, \ell}\left(\widetilde{v}_{1}, \widetilde{v}_{1}\right)|\zeta|^{c-c^{0}}$ and $h_{c, \ell}^{0}\left(\widetilde{v}_{2}, \widetilde{v}_{2}\right)=h_{c^{0}, \ell}\left(\widetilde{v}_{2}, \widetilde{v}_{2}\right)|\zeta|^{c^{0}-c}$ on $\{|\zeta| \geq 1\}$.

- $\left|\widetilde{v}_{1} \wedge \widetilde{v}_{2}\right|_{h_{c, \ell}^{0}}=|\zeta|^{\ell}$.

- We have $\lim _{c \rightarrow c^{0}} h_{c, \ell}^{0}=h_{c^{0}, \ell}^{0}$ in the $C^{\infty}$-sense on any compact subset in $\mathbb{C}$.

Note that the conditions are compatible on $\{|\zeta| \geq 1\}$. We fix a $C^{\infty}$-Kähler metric $g_{\mathbb{P}^{1}}$ of $\mathbb{P}^{1}$.

Lemma 3.16 We have a constant $C>0$ such that the following holds on $\mathbb{P}^{1} \backslash\{\infty\}$ for any $c \in U$ :

$$
\left|R\left(h_{c, \ell}^{0}\right)\right|_{h_{c, \ell}^{0}, g_{\mathbb{P} 1}} \leq C, \quad\left|\left[\widetilde{\theta}, \widetilde{\theta}_{h_{c, \ell}^{0}}^{\dagger}\right]\right|_{h_{c, \ell}^{0}, g_{\mathbb{P} 1}} \leq C
$$

Proof In the proof, $C_{i}$ and $\epsilon_{i}$ are positive constants, which are independent of $c$. It is enough to consider the issue on $\{|\zeta| \geq 1\}$. The estimate for $\left[\widetilde{\theta}, \widetilde{\theta}_{h_{c, \ell}^{\dagger}}^{\dagger}\right]$ follows from $(26)$ and the construction of $h_{c, \ell}^{0}$. Let us study the estimate for $R\left(h_{c, \ell}^{0}\right)$.

Let $H$ be the $M_{2}(\mathbb{C})$-valued function on $\{|\zeta| \geq 1\}$ given by $H_{i j}=h_{c^{0}, \ell}\left(\widetilde{v}_{i}, \widetilde{v}_{j}\right)$. By Lemma 3.13, we have positive constants $C_{1}$ and $\epsilon_{1}$ such that the following holds:

$$
\left|\partial H_{12}\right|_{g_{\mathbb{P} 1}} \leq C_{1} \exp \left(-\epsilon_{1}|\eta|^{-m-1}\right), \quad\left|\partial H_{21}\right|_{g_{\mathbb{P} 1}} \leq C_{1} \exp \left(-\epsilon_{1}|\eta|^{-m-1}\right),
$$

Set $c_{1}^{0}=c^{0}$ and $c_{2}^{0}=-c^{0}-\ell$. By Lemma 3.12, we have a positive constant $C_{2}$ such that the following holds:

$$
\left|\partial H_{i i}\right|_{g_{\mathbb{P} 1}} \leq C_{2}|\eta|^{-2 c_{i}^{0}-1}(i=1,2)
$$


Let $\Gamma_{c}$ be the $M_{2}(\mathbb{C})$-valued function given as $\Gamma_{c, 11}=|\zeta|^{c-c_{0}}, \Gamma_{c, 22}=|\zeta|^{c_{0}-c}$, and $\Gamma_{c, i j}=0(i \neq j)$. Then, $R\left(h_{c, \ell}^{0}\right)$ on $\{|\zeta| \geq 1\}$ is represented by the following matrix with respect to the frame $\left(\widetilde{v}_{1}, \widetilde{v}_{2}\right)$ :

$$
\begin{aligned}
\bar{\partial}\left(\left(\Gamma_{c} \bar{H} \Gamma_{c}\right)^{-1} \partial\left(\Gamma_{c} \bar{H} \Gamma_{c}\right)\right)=\bar{\partial}\left(\Gamma_{c}^{-1} \bar{H}^{-1}\right) & \cdot \Gamma_{c}^{-1} \partial \Gamma \cdot \bar{H} \Gamma_{c}-\Gamma_{c}^{-1} \bar{H}^{-1}\left(\Gamma_{c}^{-1} \partial \Gamma_{c}\right) \bar{\partial}(\bar{H} \Gamma) \\
& +\bar{\partial} \Gamma_{c}^{-1}\left(\bar{H}^{-1} \partial \bar{H}\right) \Gamma_{c}-\Gamma_{c}^{-1} \bar{H}^{-1} \partial \bar{H} \cdot \bar{\partial} \Gamma_{c}+\Gamma_{c}^{-1} \bar{\partial}\left(\bar{H}^{-1} \partial \bar{H}\right) \cdot \Gamma_{c}
\end{aligned}
$$

Because $\left|R\left(h_{c^{0}, \ell}\right)\right|_{h_{c^{0}, \ell}, g_{\mathbb{P}}} \leq C_{3} \exp \left(-\epsilon_{3}|\eta|^{-m-1}\right)$, we have

$$
\left|\bar{\partial}\left(\bar{H}^{-1} \partial \bar{H}\right)\right|_{g_{\mathbb{P} 1}} \leq C_{4} \exp \left(-\epsilon_{4}|\eta|^{-m-1}\right)
$$

and hence $\left|\Gamma_{c}^{-1} \bar{\partial}\left(\bar{H}^{-1} \partial \bar{H}\right) \cdot \Gamma_{c}\right|_{g_{\mathbb{P} 1}} \leq C_{5} \exp \left(-\epsilon_{5}|\eta|^{-m-1}\right)$. We also have

$$
\bar{\partial}\left(\Gamma_{c}^{-1} \bar{H}^{-1}\right) \cdot \Gamma_{c}^{-1} \partial \Gamma_{c} \cdot \bar{H} \Gamma_{c}-\Gamma_{c}^{-1} \bar{H}^{-1}\left(\Gamma_{c}^{-1} \partial \Gamma_{c}\right) \bar{\partial}\left(\bar{H} \Gamma_{c}\right)=-\left[\left(\bar{H} \Gamma_{c}\right)^{-1} \bar{\partial}\left(\bar{H} \Gamma_{c}\right),\left(\bar{H} \Gamma_{c}\right)^{-1}\left(\Gamma_{c}^{-1} \partial \Gamma_{c}\right) \bar{H} \Gamma_{c}\right]
$$

Because the off-diagonal part of $\bar{H} \Gamma_{c}$ and $\bar{\partial}\left(\bar{H} \Gamma_{c}\right)$ are dominated by $C_{6} \exp \left(-\epsilon_{6}|\eta|^{-m-1}\right)$, the term (32) is dominated by $C_{7} \exp \left(-\epsilon_{7}|\eta|^{-m-1}\right) d \zeta d \bar{\zeta}$. Similarly,

$$
\bar{\partial} \Gamma_{c}^{-1}\left(\bar{H}^{-1} \partial \bar{H}\right) \Gamma_{c}-\Gamma_{c}^{-1} \bar{H}^{-1} \partial \bar{H} \cdot \bar{\partial} \Gamma_{c}=-\left[\Gamma_{c}^{-1} \bar{\partial} \Gamma_{c}, \Gamma_{c}^{-1}\left(\bar{H}^{-1} \partial \bar{H}\right) \Gamma_{c}\right]
$$

is dominated by $C_{8} \exp \left(-\epsilon_{8}|\eta|^{-m-1}\right) d \zeta d \bar{\zeta}$. Then, the claim of the lemma follows.

We have the self-adjoint endomorphisms $k_{c}$ of $\left(\widetilde{E}_{\ell}, h_{c, \ell}^{0}\right)$ determined by $h_{c, \ell}\left(u_{1}, u_{2}\right)=h_{c, \ell}^{0}\left(k_{c} u_{1}, u_{2}\right)$. Note that $k_{c}$ are bounded with respect to $h_{c, \ell}^{0}$, although the estimate might depend on $c$ at this stage. We also remark that $\operatorname{Tr} k_{c}(P) \geq 1$ at any $P \in \mathbb{C}$. The claim of Proposition 3.15 is deduced from the following proposition.

Proposition 3.17 We have the convergence $k_{c} \longrightarrow \mathrm{id}\left(c \rightarrow c^{0}\right)$ uniformly on $\mathbb{C}$.

Proof Take a large $p>1$. Let $\left\|k_{c}\right\|_{h_{c, \ell}^{0}, g_{\mathbb{P}}, L^{p}}$ be the $L^{p}$-norm of $k_{c}$ with respect to $h_{c, \ell}^{0}$ and $g_{\mathbb{P}^{1}}$. We set $s_{c}:=k_{c} /\left\|k_{c}\right\|_{h_{c, \ell}^{0}, g_{\mathbb{P} 1}, L^{p}}$. According to [19, Lemma 3.1], we have the following inequality on $\mathbb{C}$ :

$$
\sqrt{-1} \Lambda_{g_{\mathbb{P} 1}} \bar{\partial} \partial \operatorname{Tr}\left(s_{c}\right) \leq\left|\Lambda_{g_{\mathbb{P} 1}} \operatorname{Tr}\left(\left(R\left(h_{c, \ell}^{0}\right)+\left[\widetilde{\theta}, \widetilde{\theta}_{h_{c, \ell}^{0}}^{\dagger}\right]\right) s_{c}\right)\right|
$$

We recall the following general lemma, which is a variant of [20, Lemma 2.2].

Lemma 3.18 Let $\varphi$ and $g$ be bounded $\mathbb{R}$-valued $C^{\infty}$-functions on a punctured disc $\{x \in \mathbb{C}|0<| x \mid<1\}$. Suppose that $-\partial_{x} \partial_{\bar{x}} \varphi \leq g$ holds on $\{x \in \mathbb{C}|0<| x \mid<1\}$. Then, the inequality holds on $\{x \in \mathbb{C}|| x \mid<1\}$ in the sense of distributions.

Proof We give only an outline of the proof. We take a $C^{\infty}$-function $\rho: \mathbb{R} \longrightarrow \mathbb{R}_{\geq 0}$ satisfying $\rho(t)=1(t \leq 1)$ and $\rho(t)=0(t \geq 2)$. For any $N>0$, we set $\chi_{N}(x)=\rho\left(-N^{-1} \log |x|\right)$. Note that $\partial \chi_{N}, \bar{\partial} \chi_{N}$ and $\partial \bar{\partial} \chi_{N}$ are bounded with respect to the Poincaré metric $\left(\log |x|^{2}\right)^{-1}|x|^{-2} d x d \bar{x}$, which are uniformly for $N$.

Let $f$ be any $\mathbb{R}_{\geq 0}$-valued test function on $\{|x|<1\}$. The claim of the lemma is the following inequality:

$$
\int_{|x|<1}-\varphi \partial_{x} \partial_{\bar{x}} f|d x d \bar{x}| \leq \int_{|x|<1} g \cdot f|d x d \bar{x}|
$$

By the assumption, we have $\int_{|x|<1}-\varphi \partial_{x} \partial_{\bar{x}}\left(\chi_{N} f\right)|d x d \bar{x}| \leq \int_{|x|<1} g \cdot \chi_{N} f|d x d \bar{x}|$. It is enough to prove that $\lim _{N \rightarrow \infty} \int_{|x|<1} \varphi\left(\partial \bar{\partial}\left(\chi_{N} f\right)-\chi_{N} \partial \bar{\partial}(f)\right)=0$. It follows from the uniform boundedness of $\partial \chi_{N}, \bar{\partial} \chi_{N}$ and $\partial \bar{\partial} \chi_{N}$ with respect to the Poincaré metric.

In particular, the inequality (33) holds on $\mathbb{P}^{1}$. The right hand side of (33) is uniformly bounded in $L^{p}$. We can find $L_{2}^{p}$-functions $M_{c}$ on $\mathbb{P}^{1}$ and constants $C_{i}>0(i=10,11)$ such that the following holds for any $c \in U$ :

$$
\Lambda_{g_{\mathbb{P} 1}} \bar{\partial} \partial\left(\operatorname{Tr}\left(s_{c}\right)-M_{c}\right) \leq C_{10}, \quad \sup \left|M_{c}\right| \leq C_{11}
$$


By [19, Proposition 2.1], we can take constants $C_{i}>0(i=12,13)$ such that the following holds for any $c \in U$ :

$$
\sup _{\mathbb{P}^{1}}\left(\operatorname{Tr}\left(s_{c}\right)-M_{c}\right) \leq C_{12} \int_{\mathbb{P}^{1}}\left|\operatorname{Tr}\left(s_{c}\right)-M_{c}\right| \mathrm{dvol}_{\mathbb{P}^{1}} \leq C_{13}
$$

Hence, we can take a constant $C_{14}>0$ such that the following holds for any $c \in U$ :

$$
\sup _{\mathbb{P}^{1}}\left|s_{c}\right| \leq C_{14}
$$

Again, according to [19, Proposition 3.1], we have

$$
\sqrt{-1} \Lambda_{g_{\mathbb{P} 1}} \bar{\partial} \partial \operatorname{Tr}\left(s_{c}\right)=\sqrt{-1} \Lambda_{g_{\mathbb{P} 1}} \operatorname{Tr}\left(s_{c}\left(R\left(h_{c, \ell}^{0}+\left[\widetilde{\theta}, \widetilde{\theta}_{h_{c, \ell}^{0}}^{\dagger}\right]\right)\right)\right)-\left|s_{c}^{-1 / 2}(\bar{\partial}+\widetilde{\theta}) s_{c}\right|_{h_{c, \ell}^{0}, g_{\mathbb{P} 1}}^{2} .
$$

Hence, we obtain the boundedness of $\left\|s_{c}^{-1 / 2}(\bar{\partial}+\widetilde{\theta}) s_{c}\right\|_{h_{c, \ell}^{0}, g_{\mathbb{P} 1}, L^{2}}(c \in U)$. Moreover, we have

$$
\left\|s_{c}^{-1 / 2}(\bar{\partial}+\widetilde{\theta}) s_{c}\right\|_{h_{c, \ell}^{0}, g_{\mathbb{P} 1}, L^{2}} \rightarrow 0 \quad\left(c \rightarrow c^{0}\right)
$$

Because $s_{c}^{1 / 2}$ is uniformly bounded with respect to $h_{c, \ell}^{0}$, we obtain the boundedness of $\left\|(\bar{\partial}+\widetilde{\theta}) s_{c}\right\|_{h_{c, \ell}^{0}, g_{\mathbb{P}}, L^{2}}$ $(c \in U)$. We also have $\left\|(\bar{\partial}+\widetilde{\theta}) s_{c}\right\|_{h_{c, \ell}^{0}, g_{\mathbb{P} 1}, L^{2}} \rightarrow 0\left(c \rightarrow c^{0}\right)$. In particular, we obtain the uniform boundedness of $s_{c}$ in $L_{1}^{2}$ with respect to $h_{c, \ell}^{0}$.

We take any subsequence $s_{c^{i}}$ which is weakly convergent in $L_{1}^{2}$ locally on $\mathbb{P}^{1} \backslash\{\infty\}$. Let $s_{\infty}$ denote the limit. The sequence $s_{c^{i}}$ converges to $s_{\infty}$ almost everywhere. By the uniform boundedness (34), we have the boundedness of $s_{\infty}$. It also implies $\left\|s_{\infty}\right\|_{L^{p}}=\lim \left\|s_{c^{i}}\right\|_{L^{p}}=1$. In particular, $s_{\infty} \neq 0$. By the construction, we have $(\bar{\partial}+\widetilde{\theta}) s_{\infty}=0$. Hence, $s_{\infty}$ gives a non-zero endomorphism of the stable filtered Higgs bundle $\left(\mathcal{P}_{*}^{c^{0}} \widetilde{E}_{\ell}, \widetilde{\theta}\right)$. It implies that $s_{\infty}$ is the multiplication of a non-zero complex number. In particular, $\operatorname{det}\left(s_{\infty}\right) \neq 0$. It implies that $\left\|k_{c^{i}}\right\|_{L^{p}, h_{c, \ell}^{0}, g_{\mathbb{P} 1}}$ are bounded. We obtain that $\left\|k_{c}\right\|_{L^{p}, h_{c, \ell}^{0}, g_{\mathbb{P} 1}}(c \in U)$ are bounded.

It implies that the sequence $k_{c}(c \in U)$ are bounded in $L_{1}^{2}$. We also have $C_{15}>0$ such that $\sup _{\mathbb{P}^{1}}\left|k_{c}\right|<C_{15}$ $(c \in U)$. We take any subsequence $k_{c^{i}}$ which is weakly convergent in $L_{1}^{2}$ locally on $\mathbb{P}^{1} \backslash\{\infty\}$. Then, the limit $k_{\infty}$ is the multiplication of a non-zero positive number. Because $\operatorname{det}\left(k_{c}\right)=1$ for any $c$, we obtain that $k_{\infty}=\mathrm{id}$. It implies that $k_{c}(c \in U)$ is weakly convergent to id in $L_{1}^{2}$ locally on $\mathbb{P}^{1} \backslash\{\infty\}$.

By using [19, Proposition 3.1], we obtain $\sqrt{-1} \Lambda_{g_{\mathbb{P} 1}} \bar{\partial} \partial\left(\operatorname{Tr}\left(k_{c}\right)-2\right) \leq C_{16}(c \in U)$ for a constant $C_{16}>0$. By [19, Proposition 2.1], we obtain $\sup _{\mathbb{P}^{1}}\left(\operatorname{Tr}\left(k_{c}\right)-2\right) \leq C_{17} \int_{\mathbb{P}^{1}}\left|\operatorname{Tr}\left(k_{c}\right)-2\right| \operatorname{dvol}_{\mathbb{P}^{1}}(c \in U)$ for a constant $C_{17}>0$. Note that we always have $\operatorname{Tr}\left(k_{c}\right)-2 \geq 0$, and $\operatorname{Tr}\left(k_{c}\right)-2=0$ implies that $k_{c}=$ id. Then, we obtain the uniform convergence $\operatorname{Tr}\left(k_{c}\right)-2 \rightarrow 0\left(c \rightarrow c_{0}\right)$. It implies the uniform convergence $k_{c} \rightarrow \mathrm{id}\left(c \rightarrow c_{0}\right)$. Thus, the claims of Proposition 3.17 and Proposition 3.15 are proved.

\subsubsection{Behaviour of $b_{c}$ when $c \rightarrow 0$}

Recall that we have the following description on $\{|\zeta|>1\}$ :

$$
\log \left|\widetilde{v}_{1}\right|_{h_{c, \ell}}=c \log |\zeta|+\log b_{c}+\rho_{1, c}, \quad \log \left|\widetilde{v}_{2}\right|_{h_{c, \ell}}=-(\ell+c) \log |\zeta|-\log b_{c}+\rho_{2, c}
$$

According to Proposition 3.10, we have $\left|\rho_{i, c}\right| \leq C_{30} \exp \left(-\epsilon_{30}|\zeta|^{m+1}\right)(i=1,2)$ for positive constants $C_{30}$ and $\epsilon_{30}$ which are independent of $c$.

Proposition 3.19 When $c \rightarrow 0$, we have $b_{c} \rightarrow \infty$. We also have the uniform convergences $\rho_{i, c} \longrightarrow 0(i=1,2)$.

Proof First, let us study the convergence of $\rho_{i, c}$. We begin with a preliminary. We set $Y_{0}:=\mathbb{C}_{w} \times \mathbb{P}^{1}$ and $D_{0}:=\mathbb{C}_{w} \times\{\infty\}$. Let $p: Y_{0} \longrightarrow \mathbb{P}^{1}$ be the projection. We have the locally free $\mathcal{O}_{Y_{0}}\left(* D_{0}\right)$-module $p^{*}\left(L_{1} \oplus L_{2}\right)$. Let $\bar{v}_{i}$ be the pull back of $\widetilde{v}_{i}$. We have the $\mathcal{O}_{Y}\left(* D_{Y}\right)$-submodule $\bar{E}_{\ell} \subset p^{*}\left(L_{1} \oplus L_{2}\right)$ generated by $\bar{e}_{1}=\bar{v}_{1}+w \cdot \bar{v}_{2}$ and $\bar{e}_{2}=\zeta^{\ell} \bar{v}_{2}$. 
The restriction of $\bar{E}_{\ell}$ to $\{w\} \times \mathbb{P}^{1}$ is denoted by $\bar{E}_{\ell, w}$. For each $w$, we may naturally regard $\bar{E}_{\ell, w}$ as a subsheaf of $\widetilde{V}=L_{1} \oplus L_{2}$. We have $\widetilde{\theta}\left(\bar{E}_{\ell, w}\right) \subset \bar{E}_{\ell, w} \otimes \Omega_{\mathbb{P}^{1}}^{1}$. So, we have the family of Higgs bundles $\left(\bar{E}_{\ell, w}, \widetilde{\theta}\right)$. The restrictions of $\bar{v}_{i}$ and $\bar{e}_{i}$ to $\{w\} \times \mathbb{P}^{1}$ are denoted by $\bar{v}_{i, w}$ and $\bar{e}_{i, w}$, respectively. We have

$$
\begin{gathered}
\widetilde{\theta}\left(\bar{v}_{1, w}, \bar{v}_{2, w}\right)=\left(\bar{v}_{1, w}, \bar{v}_{2, w}\right)\left(\begin{array}{cc}
\alpha \zeta^{m} d \zeta & 0 \\
0 & -\alpha \zeta^{m} d \zeta
\end{array}\right), \\
\widetilde{\theta}\left(\bar{e}_{1, w}, \bar{e}_{2, w}\right)=\left(\bar{e}_{1, w}, \bar{e}_{2, w}\right)\left(\begin{array}{cc}
\alpha \zeta^{m} d \zeta & 0 \\
-2 w \alpha \zeta^{m-\ell} d \zeta & -\alpha \zeta^{m} d \zeta
\end{array}\right) .
\end{gathered}
$$

We have a natural isomorphism $\bar{E}_{\ell, 0} \simeq L_{1} \oplus \zeta^{\ell} L_{2}$. For $w \neq 0$, we pick $w^{1 / 2}$, then we have the isomorphism $\Phi_{w}:\left(\bar{E}_{\ell, w}, \widetilde{\theta}\right) \simeq\left(\widetilde{E}_{\ell}, \widetilde{\theta}\right)$ given by $w^{-1 / 2} \bar{e}_{1, w} \longleftrightarrow e_{1}$ and $w^{1 / 2} \bar{e}_{2, w} \longleftrightarrow e_{2}$, i.e., $w^{-1 / 2} \bar{v}_{1, w} \longleftrightarrow v_{1}$ and $w^{1 / 2} \bar{v}_{2, w} \longleftrightarrow v_{2}$.

We take a $C^{\infty}$-metric $\bar{h}$ of $\bar{E}_{\ell \mid \mathbb{C}_{w} \times \mathbb{C}_{\zeta}}$ satisfying the following conditions:

- On $\mathbb{C}_{w} \times\{|\zeta| \geq 1\}$, we have $\bar{h}\left(\bar{v}_{1}, \bar{v}_{1}\right)=|\zeta|^{-2|w|}, \bar{h}\left(\bar{v}_{2}, \bar{v}_{2}\right)=|\zeta|^{-2(\ell-|w|)}$, and $\bar{h}\left(\bar{v}_{1}, \bar{v}_{2}\right)=0$.

- On $\{w=0\} \times \mathbb{C}_{\zeta}$, we have $\bar{h}\left(\bar{e}_{1,0}, \bar{e}_{1,0}\right)=1, \bar{h}\left(\bar{e}_{2,0}, \bar{e}_{2,0}\right)=1$, and $\bar{h}\left(\bar{e}_{1,0}, \bar{e}_{2,0}\right)=0$.

The restriction of $\bar{h}$ to $\{w\} \times \mathbb{P}^{1}$ is denoted by $\bar{h}_{w}$. We take a small $\delta>0$, and consider $U_{w}:=\{|w| \leq \delta\}$. We have the following uniform boundedness on $\mathbb{C}_{\zeta}$ :

$$
\left|R\left(\bar{h}_{w}\right)+\left[\widetilde{\theta}, \widetilde{\theta}_{\bar{h}_{w}}^{\dagger}\right]\right|_{\bar{h}_{w}, g_{\mathbb{P} 1}} \leq C_{31} \quad\left(w \in U_{w}\right) .
$$

Moreover, we have the uniform convergence $\lim _{w \rightarrow 0}\left(R\left(\bar{h}_{w}\right)+\left[\widetilde{\theta}, \widetilde{\theta}_{\bar{h}_{w}}^{\dagger}\right]\right)=0$.

For any $c$ satisfying $-\delta<c<0$, we have the self-adjoint endomorphism $k_{c}$ of $\left(\bar{E}_{\ell,-c}, \bar{h}_{-c}\right)$ determined by $\Phi_{-c}^{*} h_{c, \ell}\left(u_{1}, u_{2}\right)=\bar{h}_{-c}\left(k_{c} u_{1}, u_{2}\right)$. Take a large $p>1$. Let $\left\|k_{c}\right\|_{\bar{h}_{-c}, g_{\mathbb{P}}, L^{p}}$ be the $L^{p}$-norm of $k_{c}$ with respect to $\bar{h}_{-c}$ and $g_{\mathbb{P}^{1}}$. We set $s_{c}:=k_{c} /\left\|k_{c}\right\|_{\bar{h}_{-c}, g_{\mathbb{1} 1}, L^{p}}$.

Suppose that $\left|\rho_{1, c}\right|+\left|\rho_{2, c}\right|$ is not uniformly convergent to 0 when $c \rightarrow 0$, and we shall deduce a contradiction. Under the assumption, we have a positive number $\delta>0$ and a subsequence $c^{j} \rightarrow 0$ such that

$$
\sup _{|\zeta|>1}\left(\left|\rho_{1, c^{j}}\right|+\left|\rho_{2, c^{j}}\right|\right) \geq \delta \text {. }
$$

By the argument in the proof of Proposition 3.17, we can assume that $s_{c j}$ weakly converges to a non-zero endomorphism $s_{\infty}$ of $\bar{E}_{\ell, 0}$ in $L_{1}^{2}$ locally on $\mathbb{P}^{1} \backslash\{\infty\}$, such that (i) $(\bar{\partial}+\widetilde{\theta}) s_{\infty}=0$, (ii) $s_{\infty}$ is bounded with respect to $\bar{h}_{0}$. So, it gives an endomorphism of the poly-stable parabolic Higgs bundle $\left(\mathcal{P}_{*}^{\bar{h}_{0}} \bar{E}_{0}, \widetilde{\theta}\right)$. We obtain that $s_{\infty}=\alpha_{1} \operatorname{id}_{L_{1}} \oplus \alpha_{2} \operatorname{id}_{\zeta^{\ell} L_{2}}$. Here, $\alpha_{i}$ are non-negative real numbers, and $\left(\alpha_{1}, \alpha_{2}\right) \neq(0,0)$. Suppose that $\alpha_{1} \neq 0$. We have the following uniform convergence on any compact subset in $\mathbb{C}$.

$$
\lim _{j \rightarrow \infty}\left(h_{c^{j}, \ell}\left(\widetilde{v}_{1}, \widetilde{v}_{1}\right)|\zeta|^{-2 c^{j}}\left\|k_{c^{j}}\right\|_{h_{-c^{j}}, g_{\mathbb{P} 1}, L^{p}}^{-1} \alpha_{1}^{-1}\right)=1
$$

It implies that $\rho_{1, c^{j}}$ is convergent to 0 on any compact subset in $\{|\zeta| \geq 1\}$. Together with the uniform estimate $\left|\rho_{1, c^{j}}\right| \leq C_{30} \exp \left(-\epsilon_{30}|\zeta|^{m+1}\right)$, we obtain the uniform convergence $\lim _{j \rightarrow \infty} \sup _{|\zeta|>1}\left|\rho_{1, c^{j}}\right|=0$. We have $\left|\widetilde{v}_{1} \wedge \widetilde{v}_{2}\right|_{h_{c, \ell}}=|\zeta|^{-\ell}$. By (26), we have the following estimate:

$$
\left.|| \widetilde{v}_{1}\right|_{h_{c, \ell}} \cdot\left|\widetilde{v}_{2}\right|_{h_{c, \ell}}-|\zeta|^{-\ell} \mid \leq C_{32} \exp \left(-\epsilon_{32}|\zeta|^{m+1}\right)
$$

Here, the constants $C_{32}$ and $\epsilon_{32}$ are independent of $c$. Then, we can deduce the uniform convergence $\rho_{2, c^{j}} \rightarrow 0$. Hence, we obtain $\lim _{j \rightarrow \infty} \sup _{|\zeta|>1}\left|\rho_{i, c^{j}}\right|=0(i=1,2)$ in the case $\alpha_{1} \neq 0$. Similarly, we can deduce the uniform convergences $\lim _{j \rightarrow \infty} \sup _{|\zeta|>1}\left|\rho_{i, c^{j}}\right|=0(i=1,2)$ in the case $\alpha_{2} \neq 0$. But, it contradicts with (35). Thus, we can conclude the uniform convergences $\lim _{c \rightarrow 0} \rho_{i, c}=0(i=1,2)$. 
Let us study the divergence of $b_{c}$. By the convergence of $\rho_{i, c}$, we have the following on $\{|\zeta| \geq 1\}$ :

$$
\lim _{c \rightarrow 0}\left(b_{c}^{-1}\left|\widetilde{v}_{1}\right|_{h_{c, \ell}}\right)=1, \quad \lim _{c \rightarrow 0}\left(b_{c}\left|\widetilde{v}_{2}\right|_{h_{c, \ell}}\right)=|\zeta|^{-\ell} .
$$

Suppose that there exists a subsequence $c^{i} \rightarrow 0$ such that $b_{c^{i}}$ are bounded, and we shall deduce a contradiction. We may assume the convergence $b_{c^{i}} \rightarrow \check{b}$. We shall give a detailed argument in the case $\breve{b}=0$. Later, we shall sketch the argument for the simpler case $\check{b} \neq 0$.

We set $Y_{1}:=\mathbb{C}_{x} \times \mathbb{P}^{1}$ and $D_{1}:=\mathbb{C}_{x} \times\{\infty\}$. Let $p_{1}: Y_{1} \longrightarrow \mathbb{P}^{1}$ be the projection. We have the locally free $\mathcal{O}_{Y_{1}}\left(* D_{1}\right)$-module $p_{1}^{*}\left(L_{1} \oplus L_{2}\right)$. The pull back of $v_{i}$ are denoted by $\widehat{v}_{i}$. Let $\widehat{E}_{\ell} \subset p_{1}^{*}\left(L_{1} \oplus L_{2}\right)$ generated by $\widehat{f}_{1}=x \widehat{v}_{1}+\widehat{v}_{2}$ and $\widehat{f}_{2}=\zeta^{\ell} \widehat{v}_{1}$. The restriction of $\widehat{E}_{\ell}$ to $\{x\} \times \mathbb{P}^{1}$ is denoted by $\widehat{E}_{\ell, x}$. The restriction of $\widehat{v}_{i}$ and $\widehat{f}_{i}$ to $\{x\} \times \mathbb{P}^{1}$ are denoted by $\widehat{v}_{i, x}$ and $\widehat{f}_{i, x}$. We have

$$
\begin{gathered}
\tilde{\theta}\left(\widehat{v}_{1, x}, \widehat{v}_{2, x}\right)=\left(\widehat{v}_{1, x}, \widehat{v}_{2, x}\right)\left(\begin{array}{cc}
\alpha \zeta^{m} d \zeta & 0 \\
0 & -\alpha \zeta^{m} d \zeta
\end{array}\right) \\
\tilde{\theta}\left(\widehat{f}_{1, x}, \widehat{f}_{2, x}\right)=\left(\widehat{f}_{1, x}, \widehat{f}_{2, x}\right)\left(\begin{array}{cc}
\alpha \zeta^{m} d \zeta & 0 \\
2 x \alpha \zeta^{m-\ell} d \zeta & -\alpha \zeta^{m} d \zeta
\end{array}\right)
\end{gathered}
$$

We have $\widehat{E}_{\ell, 0} \simeq \zeta^{\ell} L_{1} \oplus L_{2}$. For $x \neq 0$, taking $x^{1 / 2}$, we have the isomorphism $\Psi_{x}:\left(\widehat{E}_{\ell, x}, \widetilde{\theta}\right) \simeq\left(\widetilde{E}_{\ell}, \widetilde{\theta}\right)$ given by the correspondences $x^{-1 / 2} \widehat{f}_{1, x} \longleftrightarrow v_{1}+v_{2}$ and $x^{1 / 2} \widehat{f}_{2, x} \longleftrightarrow \zeta^{\ell} v_{1}$, i.e., $x^{1 / 2} \widehat{v}_{1} \longleftrightarrow v_{1}$ and $x^{-1 / 2} \widehat{v}_{2} \longleftrightarrow v_{2}$.

We have the isomorphisms of holomorphic vector bundles $\Upsilon_{x_{i}}: \widehat{E}_{\ell, 0} \simeq \widehat{E}_{\ell, x_{i}}$ given by $\Upsilon_{x_{i}}\left(\widehat{f}_{j, 0}\right)=\widehat{f}_{j, x}$. We shall implicitly identify the vector bundles $\widehat{E}_{\ell, 0}$ and $\widehat{E}_{\ell, x_{i}}$ by $\Upsilon_{x_{i}}$ in the following argument.

Let $x_{i}:=b_{c^{i}}^{2}$. By the assumption, we have $\lim _{i \rightarrow \infty} x_{i}=0$. We obtain the harmonic metrics $\Psi_{x_{i}}^{*} h_{c^{i}, \ell}$ on $\left(\widehat{E}_{\ell, x_{i}}, \theta\right)$. We take a family of $C^{\infty}$-Hermitian metrics $h_{x_{i}}^{0}$ of $\widehat{E}_{\ell, x_{i} \mid \mathbb{C}_{\zeta}}$ satisfying the following conditions.

- $h_{0}^{0}\left(\widehat{v}_{1,0}, \widehat{v}_{1,0}\right)=1, h_{0}^{0}\left(\widehat{v}_{2,0}, \widehat{v}_{2,0}\right)=|\zeta|^{-2 \ell}$, and $h_{0}^{0}\left(\widehat{v}_{1,0}, \widehat{v}_{2,0}\right)=0$.

- $\Upsilon_{x_{i}}^{*} h_{x_{i}}^{0} \rightarrow h_{0}^{0}$ in the $C^{\infty}$-sense on any compact subset in $\mathbb{C}_{\zeta}$.

- We have $h_{x_{i}}^{0}\left(\widehat{v}_{1, x}, \widehat{v}_{1, x}\right)=|\zeta|^{2 c^{i}}, h_{x_{i}}^{0}\left(\widehat{v}_{2, x}, \widehat{v}_{2, x}\right)=|\zeta|^{-2 c^{i}-2 \ell}$ and $h_{x_{i}}^{0}\left(\widehat{v}_{1, x}, \widehat{v}_{2, x}\right)=0$ on $\{|\zeta|>1\}$.

Let $k_{i}$ be the self-adjoint endomorphism of $\left(\widehat{E}_{\ell, x_{i}}, h_{x_{i}}^{0}\right)$ determined by $\Psi_{x_{i}}^{*} h_{c^{i}, \ell}=h_{x_{i}}^{0} \cdot k_{i}$. By (36), we have the convergence of $\Upsilon_{x_{i}}^{*} k_{i}$ to the identity on any compact subset in $\{|\zeta|>1\}$. We have the convergence of $\Upsilon_{x_{i}}^{*} k_{i}^{-1}$ to the identity on any compact subset in $\{|\zeta|>1\}$.

Let us study the convergence of $\Upsilon_{x_{i}}^{*} k_{i}$ and $\Upsilon_{x_{i}}^{*} k_{i}^{-1}$ on $\{|\zeta|<2\}$. We have the following uniform estimate:

$$
\left|R\left(h_{c^{i}, \ell}\right)\right|_{h_{c^{i}, \ell}, g_{\mathbb{P} 1}} \leq C_{42}
$$

Hence, we have a constant $C_{43}>0$ which is independent of $c^{i}$ such that $-\partial_{\zeta} \partial_{\bar{\zeta}} \log \left|\widehat{f}_{1}\right|_{h_{c^{i}, \ell}} \leq C_{43}$ on $\{|\zeta|<2\}$. Namely, we have

$$
-\partial_{\zeta} \partial_{\bar{\zeta}}\left(\log \left|\widehat{f}_{1}\right|_{h_{c^{i}, \ell}}-C_{43}|\zeta|^{2}\right) \leq 0
$$

We have already known the uniform boundedness of $\left|\widehat{f}_{1}\right|_{h_{c^{i}, \ell}}$ on $\{1<|\zeta|<2\}$. Then, we obtain the uniform boundedness of $\left|\widehat{f}_{1}\right|_{h_{c^{i}, \ell}}$ on $\{|\zeta|<2\}$. Similarly, we obtain the uniform boundedness of $\left|\widehat{f}_{2}\right|_{h_{c^{i}, \ell}}$ on $\{|\zeta|<2\}$. Hence, $k_{i}$ and $k_{i}^{-1}$ are uniformly bounded on $\{|\zeta|<2\}$.

Then, we may assume that the sequence $\Upsilon_{x_{i}}^{*} k_{i}$ is weakly convergent to $k_{\infty}$ in $L_{2}^{p}$ on any compact subset in $\mathbb{C}_{\zeta}$. We have the convergence of $\Upsilon_{x_{i}}^{*} k_{i}^{-1}$ to $k_{\infty}^{-1}$. We obtain a harmonic metric $h_{\infty}=h_{0}^{0} \cdot k_{\infty}$. By the construction, we have $\mathcal{P}_{*}^{0} \widehat{E}_{\ell, 0} \subset \mathcal{P}_{*}^{h} \widehat{E}_{\ell, 0}$. Because $\operatorname{deg}\left(\mathcal{P}_{*}^{0} \widehat{E}_{\ell, 0}\right)=\operatorname{deg}\left(\mathcal{P}_{*}^{h} \widehat{E}_{\ell, 0}\right)=0$, we obtain $\mathcal{P}_{*}^{0} \widehat{E}_{\ell, 0}=\mathcal{P}_{*}^{h} \widehat{E}_{\ell, 0}$ by Lemma 3.6. But, $\left(\mathcal{P}_{*}^{0} \widehat{E}_{\ell, 0}, \widetilde{\theta}\right)$ is not poly-stable because $\mathcal{P}_{0}^{0} L_{2} \simeq \mathcal{O}_{\mathbb{P}^{1}}(\ell)$ and $\operatorname{deg} \mathcal{P}_{0}^{0} L_{2}=\ell>0$. Hence, we have deduced a contradiction from the assumption $b_{c^{i}} \rightarrow 0$. 
Let us give a sketch of the argument in the case $\lim b_{c^{i}}=\breve{b} \neq 0$. Under the assumption, the sequence $h_{c^{i}, \ell}$ is convergent on $\{|\zeta| \geq 1\}$. As in the previous case, by taking a subsequence, we may assume that the sequence $h_{c^{i}, \ell}$ is weakly convergent in $L_{2}^{p}$ on any compact subset in $\mathbb{C}_{\zeta}$, and the limit $h_{\infty}$ is a harmonic metric of $\left(\widetilde{E}_{\ell}, \theta\right)$ which is adapted to the filtered bundle $\mathcal{P}_{*}^{0} \widetilde{E}_{\ell}$. But, $\left(\mathcal{P}_{*}^{0} \widetilde{E}_{\ell}, \theta\right)$ is not poly-stable. Hence, we obtain a contradiction even in the case $\breve{b} \neq 0$.

\subsubsection{Convergence of some sequences}

Let $t_{i}$ be a sequence of positive numbers such that $t_{i} \rightarrow \infty$. According to Proposition 3.15 and Proposition 3.19, we can take a sequence of negative numbers $c_{i}$ such that $c_{i} \rightarrow 0$ and $b_{c_{i}} t_{i}^{c_{i} /(m+1)}=1$. We set $\tau_{i}:=t_{i}^{1 / 2(m+1)}$.

We use the notation in $\S 3.2 .4$. We have the isomorphisms $\Phi_{\tau_{i}}: \varphi_{\tau_{i}}^{*} \widetilde{E}_{\ell} \simeq \widetilde{E}_{\ell}$. Let $h_{i}$ denote the Hermitian metric of $\widetilde{E}_{\ell}$ induced by $\Phi_{\tau_{i}}$ and $\varphi_{\tau_{i}}^{*} h_{c_{i}, \ell}$. Take any $T>0$. We have the following estimates on $\{|\zeta| \geq T\}$ for any $i$ such that $t_{i}^{1 /(m+1)} T>1$, where $C$ and $\epsilon$ are positive constants independent of $i$ and $T$ :

$$
\begin{gathered}
\left.|\log | \widetilde{v}_{1}\right|_{h_{i}}-\log \left(|\zeta|^{c_{i}} t_{i}^{\ell / 2(m+1)}\right) \mid \leq C \exp \left(-\epsilon|\zeta|^{m+1} t_{i}\right) \\
\left.|\log | \widetilde{v}_{2}\right|_{h_{i}}-\log \left(|\zeta|^{-\ell-c_{i}} t_{i}^{-\ell / 2(m+1)}\right) \mid \leq C \exp \left(-\epsilon|\zeta|^{m+1} t_{i}\right) \\
\left|h_{i}\left(\widetilde{v}_{1}, \widetilde{v}_{2}\right)\right| \leq C \exp \left(-\epsilon|\zeta|^{m+1} t_{i}\right) \cdot\left|\widetilde{v}_{1}\right|_{h_{i}} \cdot\left|\widetilde{v}_{2}\right|_{h_{i}}
\end{gathered}
$$

Let $h^{\text {lim }}$ be the Hermitian metric of $\widetilde{V}_{\mid \mathbb{C}^{*}}$ given by

$$
h^{\lim }\left(\widetilde{v}_{1}, \widetilde{v}_{1}\right)=1, \quad h^{\lim }\left(\widetilde{v}_{2}, \widetilde{v}_{2}\right)=|\zeta|^{-2 \ell}, \quad h^{\lim }\left(\widetilde{v}_{1}, \widetilde{v}_{2}\right)=0 .
$$

For any $\gamma>0$, the automorphism $\Psi_{\gamma}$ on $\widetilde{V}_{\mathbb{C}^{*}}$ is given by $\Psi_{\gamma}=\gamma \operatorname{id}_{L_{1}} \oplus \gamma^{-1} \operatorname{id}_{L_{2}}$. We define $\Psi_{\gamma}^{*} h_{i}\left(u_{1}, u_{2}\right):=$ $h_{i}\left(\Psi_{\gamma} u_{1}, \Psi_{\gamma} u_{2}\right)$. The following is clear.

Proposition 3.20 Set $\gamma_{i}:=t_{i}^{-\ell / 2(m+1)}$. Then, we have the convergence $\lim _{i \rightarrow \infty} \Psi_{\gamma_{i}}^{*} h_{i}=h^{\lim }$ on $\mathbb{C}^{*}$.

\subsection{Complement}

We use the notation in $\S 3.2$. Take $0<\kappa<1$. We take a $C^{\infty}$-function $\rho: \mathbb{C} \longrightarrow \mathbb{R}_{\geq 0}$ satisfying $\rho(\zeta)=1$ $(|\zeta| \leq 1 / 2)$ and $\rho(\zeta)=0(|\zeta| \geq 1)$. We set

$$
u_{1}:=-\left(\kappa \rho(\zeta)+|\zeta|^{2 \ell}\right)^{-1} \bar{\zeta}^{\ell} e_{2}+e_{1}, \quad u_{2}:=e_{2} .
$$

They give a $C^{\infty}$-frame of $\widetilde{E}_{\ell}$. Note that we have $u_{1}=v_{1}$ and $u_{2}=\zeta^{\ell} v_{2}$ on $\{|\zeta| \geq 1\}$.

We take a large integer $L$. Let $h_{\kappa}$ be the Hermitian metric of $\widetilde{E}_{\ell}$ determined by the following conditions:

$$
\left|u_{2}\right|_{h_{\kappa}}=\kappa^{L}, \quad\left|u_{1}\right|_{h_{\kappa}}=\kappa^{-L}, \quad h_{\kappa}\left(u_{1}, u_{2}\right)=0
$$

Let $\nabla_{\kappa}$ be the Chern connection of $\left(\widetilde{E}_{\ell}, h_{\kappa}\right)$, and let $R\left(h_{\kappa}\right)$ denote the curvature of $\nabla_{\kappa}$. Let $\widetilde{\theta}_{\kappa}^{\dagger}$ denote the adjoint of $\widetilde{\theta}$ with respect to $h_{\kappa}$. Let $\widetilde{\theta}_{\kappa}^{\dagger}$ denote the adjoint of $\widetilde{\theta}$ with respect to $h_{\kappa}$.

Lemma 3.21 On $\{|\zeta| \leq 1\}$, we have $\left|R\left(h_{\kappa}\right)\right|_{h_{\kappa}, g_{\mathrm{C}}} \leq C_{50} \kappa^{L}$ and $\left|\left[\widetilde{\theta}, \widetilde{\theta}_{\kappa}^{\dagger}\right]\right|_{h_{\kappa}, g_{\mathrm{C}}} \leq C_{50} \kappa^{L}$ for a constant $C_{50}>0$. On $\{|\zeta| \geq 1\}$, we have $R\left(h_{\kappa}\right)=\left[\widetilde{\theta}, \widetilde{\theta}_{\kappa}^{\dagger}\right]=0$.

Proof In the proof, $O\left(\kappa^{L}\right)$ denotes functions which are dominated by $C_{51} \kappa^{L}$ for a constant $C_{51}>0$. The equalities on $\{|\zeta| \geq 1\}$ are clear. Let us argue the estimates on $\{|\zeta| \leq 1\}$. We have $\bar{\partial}\left(\kappa^{-L} u_{2}\right)=0$, and

$$
\bar{\partial}\left(\kappa^{L} u_{1}\right)=-\frac{\bar{\partial}\left(\bar{\zeta}^{\ell} \rho\right) \cdot \kappa^{2 L+1}}{\left(\kappa \rho(\zeta)+|\zeta|^{2 \ell}\right)^{2}}\left(\kappa^{-L} u_{2}\right)
$$


We have

$$
\nabla_{\kappa}\left(\kappa^{L} u_{1}, \kappa^{-L} u_{2}\right)=\left(\kappa^{L} u_{1}, \kappa^{-L} u_{2}\right) A, \quad A=\left(\begin{array}{cc}
0 & \frac{\partial\left(\zeta^{\ell} \rho(\zeta)\right) \kappa^{2 L+1}}{\left(\kappa \rho(\zeta)+|\zeta|^{2 \ell}\right)^{2}} \\
\frac{-\bar{\partial}\left(\bar{\zeta}^{\ell} \rho(\zeta)\right) \kappa^{2 L+1}}{\left(\kappa \rho(\zeta)+|\zeta|^{2 \ell}\right)^{2}} & 0
\end{array}\right)
$$

It is easy to obtain the following estimate on $\{|\zeta| \leq 1\}$ :

$$
\frac{\bar{\partial}\left(\bar{\zeta}^{\ell} \rho(\zeta)\right) \kappa^{2 L+1}}{\left(\kappa \rho(\zeta)+|\zeta|^{2 \ell}\right)^{2}}=O\left(\kappa^{L}\right) d \bar{\zeta}, \quad \frac{\partial\left(\zeta^{\ell} \rho(\zeta)\right) \kappa^{2 L+1}}{\left(\kappa \rho(\zeta)+|\zeta|^{2 \ell}\right)^{2}}=O\left(\kappa^{L}\right) d \zeta
$$

We also have the following on $\{|\zeta| \leq 1\}$ :

$$
d\left(\frac{\bar{\partial}\left(\bar{\zeta}^{\ell} \rho(\zeta)\right) \kappa^{2 L+1}}{\left(\kappa \rho(\zeta)+|\zeta|^{2 \ell}\right)^{2}}\right)=O\left(\kappa^{L}\right) d \zeta d \bar{\zeta}
$$

Hence, we have the following on $\{|\zeta| \leq 1\}$ :

$$
d A+A \wedge A=O\left(\kappa^{L}\right) d \zeta d \bar{\zeta}
$$

It implies the estimate for $R\left(h_{\kappa}\right)$ on $\{|\zeta| \leq 1\}$.

The Higgs field $\widetilde{\theta}$ is represented as follows:

$$
\tilde{\theta}\left(\kappa^{L} u_{1}, \kappa^{-L} u_{2}\right)=\left(\kappa^{L} u_{1}, \kappa^{-L} u_{2}\right)\left(\begin{array}{cc}
\alpha \zeta^{m} d \zeta & 0 \\
\frac{-2 \kappa^{2 L+1} \alpha \rho(\zeta) \zeta^{m-\ell}}{\kappa \rho(\zeta)+|\zeta|^{2 \ell}} d \zeta & -\alpha \zeta^{m} d \zeta
\end{array}\right)
$$

The adjoint $\widetilde{\theta}_{\kappa}^{\dagger}$ is represented as follows:

$$
\widetilde{\theta}_{\kappa}^{\dagger}\left(\kappa^{L} u_{1}, \kappa^{-L} u_{2}\right)=\left(\kappa^{L} u_{1}, \kappa^{-L} u_{2}\right)\left(\begin{array}{cc}
\bar{\alpha} \bar{\zeta}^{m} d \bar{\zeta} & \frac{-2 \kappa^{2 L+1} \bar{\alpha} \rho(\zeta) \bar{\zeta}^{m-\ell}}{\kappa \rho(\zeta)+|\zeta|^{2 \ell}} d \bar{\zeta} \\
0 & -\bar{\alpha} \bar{\zeta}^{m} d \bar{\zeta}^{\prime}
\end{array}\right)
$$

Hence, $\left[\widetilde{\theta}, \widetilde{\theta}_{\kappa}^{\dagger}\right]$ is represented as follows:

$$
A_{2}:=\left(\begin{array}{cc}
4 \kappa^{4 L+2}|\alpha|^{2} \rho(\zeta)^{2} \frac{|\zeta|^{2(m-\ell)}}{\left(\kappa \rho(\zeta)+|\zeta|^{2 \ell}\right)^{2}} d \bar{\zeta} d \zeta & -4 \kappa^{2 L+1}|\alpha|^{2} \rho(\zeta) \frac{|\zeta|^{2 m} \zeta^{-\ell}}{\kappa \rho(\zeta)+|\zeta|^{2 \ell}} d \bar{\zeta} d \zeta \\
4 \kappa^{2 L+1}|\alpha|^{2} \rho(\zeta) \frac{|\zeta|^{2 m} \zeta^{-\ell}}{\kappa \rho(\zeta)+|\zeta|^{2 \ell}} d \bar{\zeta} d \zeta & -4 \kappa^{4 L+2}|\alpha|^{2} \rho(\zeta)^{2} \frac{|\zeta|^{2(m-\ell)}}{\left(\kappa \rho(\zeta)+|\zeta|^{2 \ell}\right)^{2}} d \bar{\zeta} d \zeta
\end{array}\right)
$$

In particular, we have the following on $\{|\zeta| \leq 1\}$ :

$$
A_{2}=O\left(\frac{\kappa^{4 L+2} \rho(\zeta)|\zeta|^{2(m-\ell)}}{\left(\kappa \rho(\zeta)+|\zeta|^{2 \ell}\right)^{2}}+\frac{\kappa^{2 L+1} \rho(\zeta)|\zeta|^{2 m-\ell}}{\kappa \rho(\zeta)+|\zeta|^{2 \ell}}\right) d \zeta d \bar{\zeta}=O\left(\kappa^{L}\right) d \zeta d \bar{\zeta}
$$

Thus, we are done.

\subsubsection{Convergence of some sequences}

Suppose that we are give a number $a>\ell / 2(m+1)$. Set $\nu:=a-\ell / 2(m+1)>0$. Let $t_{i}$ be a sequence of positive numbers such that $t_{i} \rightarrow \infty$. We set $\kappa_{i}:=t_{i}^{-\nu / L}$, for which we have $\kappa_{i} \rightarrow 0$. We have the Hermitian metric $h_{\kappa_{i}}$ of $\widetilde{E}_{\ell}$ as above.

We use the notation in $\S 3.2 .4$. We set $\tau_{i}:=t_{i}^{1 / 2(m+1)}$. We have the isomorphisms $\Phi_{\tau_{i}}: \varphi_{\tau_{i}}^{*} \widetilde{E}_{\ell} \simeq \widetilde{E}_{\ell}$. Let $h_{i}$ be the Hermitian metric of $\widetilde{E}_{\ell}$ induced by $\varphi_{\tau_{i}}^{*} h_{\kappa_{i}}$ and $\Phi_{\tau_{i}}$. 
On $\left\{|\tau| \geq \kappa_{i}\right\}$, we have the following:

$$
\left|\widetilde{v}_{1}\right|_{h_{i}}=t_{i}^{a}, \quad\left|\widetilde{v}_{2}\right|_{h_{i}}=t_{i}^{-a}|\zeta|^{-\ell}, \quad h_{i}\left(\widetilde{v}_{1}, \widetilde{v}_{2}\right)=0
$$

Let $h^{\lim }$ be the Hermitian metric of $\widetilde{V}_{\mid \mathbb{C}^{*}}$ given by

$$
h^{\lim }\left(\widetilde{v}_{1}, \widetilde{v}_{1}\right)=1, \quad h^{\lim }\left(\widetilde{v}_{2}, \widetilde{v}_{2}\right)=|\zeta|^{-2 \ell}, \quad h^{\lim }\left(\widetilde{v}_{1}, \widetilde{v}_{2}\right)=0
$$

For any $\gamma>0$, the automorphism $\Psi_{\gamma}$ on $\widetilde{V}_{\mid \mathbb{C}^{*}}$ is given by $\Psi_{\gamma}=\gamma \operatorname{id}_{L_{1}} \oplus \gamma^{-1} \operatorname{id}_{L_{2}}$. We define $\Psi_{\gamma}^{*} h_{i}\left(u_{1}, u_{2}\right):=$ $h_{i}\left(\Psi_{\gamma} u_{1}, \Psi_{\gamma} u_{2}\right)$. The following is clear.

Proposition 3.22 Set $\gamma_{i}:=t_{i}^{-a}$. Then, we have the convergence $\lim _{i \rightarrow \infty} \Psi_{\gamma_{i}}^{*} h_{i}=h^{\lim }$ on $\mathbb{C}^{*}$.

\section{Limiting configurations}

\subsection{A description of generically regular semisimple Higgs bundles of rank 2}

Let $X$ be a connected complex curve. Let $\left(E, \bar{\partial}_{E}, \theta\right)$ be a Higgs bundle on $X$ of rank 2 which is generically regular semisimple. For simplicity, we assume that $\operatorname{tr}(\theta)=0$. Let us consider the case that the spectral curve $\Sigma(E, \theta)$ is reducible, i.e., we have a holomorphic 1 -form $\omega \neq 0$ on $X$ such that $\Sigma(E, \theta)$ is the union of the images of $\omega$ and $-\omega$. Such $\omega$ is determined up to the multiplication of \pm 1 .

Remark 4.1 Suppose that $\operatorname{tr}(\theta) \neq 0$. Then, we set $\theta^{\prime}:=\theta-(\operatorname{tr} \theta / 2) \mathrm{id}_{E}$. Then, the Higgs bundle $\left(E, \bar{\partial}_{E}, \theta^{\prime}\right)$ satisfies $\operatorname{tr}\left(\theta^{\prime}\right)=0$. Hence, it is enough to study Higgs bundles such that the trace of the Higgs field is 0 .

Suppose that $\Sigma(E, \theta)$ is irreducible. We take a normalization $\varphi: \widetilde{X} \longrightarrow \Sigma(E, \theta)$. We have the induced morphism $\varphi_{1}: \widetilde{X} \longrightarrow X$. The Higgs bundle $\varphi_{1}^{*}\left(E, \bar{\partial}_{E}, \theta\right)$ satisfies the above condition. If $X$ is compact and $\left(E, \bar{\partial}_{E}, \theta\right)$ is stable, then $\varphi_{1}^{*}\left(E, \bar{\partial}_{E}, \theta\right)$ is stable or poly-stable. So, the study on the irreducible case can be reduced to the reducible case. (See also $\$ 4.3 .2$.)

Let $Z(\omega)$ denote the zero set of $\omega$. We have the decomposition of the $\mathcal{O}_{X}(* Z(\omega))$-module

$$
E \otimes \mathcal{O}_{X}(* Z(\omega))=L_{\omega}^{\prime} \oplus L_{-\omega}^{\prime}
$$

corresponding to the decomposition of the spectral curve, i.e., $\theta=\omega \cdot \operatorname{id}_{L_{\omega}^{\prime}} \oplus(-\omega) \cdot \operatorname{id}_{L_{-\omega}^{\prime}}$. Let $L_{\omega}\left(\operatorname{resp} . L_{-\omega}\right)$ denote the $\mathcal{O}_{X}$-module obtained as the image of $E$ by the induced morphism $E \longrightarrow L_{\omega}^{\prime}$ (resp. $E \longrightarrow L_{-\omega}$ ). We can regard $E$ as an $\mathcal{O}_{X}$-submodule of $L_{\omega} \oplus L_{-\omega}$.

We obtain the $\mathcal{O}_{X}$-module $\operatorname{det}\left(L_{\omega} \oplus L_{-\omega}\right) / \operatorname{det}(E)$ whose supports are contained in $Z(\omega)$. For each $P \in Z(\omega)$, let $\ell_{P}$ denote the length of the stalk of $\operatorname{det}\left(L_{\omega} \oplus L_{-\omega}\right) / \operatorname{det}(E)$ at $P$. Because we have the exact sequence

$$
0 \longrightarrow L_{\omega} \cap E \longrightarrow E \longrightarrow L_{-\omega} \longrightarrow 0
$$

we have $L_{\omega} \cap E=L_{\omega}\left(-\sum_{P \in Z(\omega)} \ell_{P} \cdot P\right)$ in $L_{\omega} \oplus L_{-\omega}$. Similarly, we have $L_{-\omega} \cap E=L_{-\omega}\left(-\sum_{P \in Z(\omega)} \ell_{P} \cdot P\right)$ in $L_{\omega} \oplus L_{-\omega}$.

Local description Let $\left(U_{P}, z\right)$ be a small holomorphic coordinate neighbourhood around $P$ satisfying $z(P)=$ 0 . Let $s_{P}$ be any frame of $\operatorname{det}(E)_{\mid U_{P}}$.

Lemma 4.2 We have local frames $v_{ \pm}$of $L_{ \pm \omega}$ around $P$ such that $(i) e_{1}=v_{+}+v_{-}$and $e_{2}=z^{\ell_{P}} v_{-}$is a frame of $E_{\mid U_{P}}$, (ii) $e_{1} \wedge e_{2}=s_{P}$.

Proof If $\ell_{P}=0$, we have $E=L_{\omega} \oplus L_{-\omega}$ on $U_{P}$, and hence the claim is clear. We shall consider the case $\ell_{P}>0$. We omit to distinguish the restriction to $U_{P}$. We take a frame $e_{2}^{\prime}$ of $z^{\ell} L_{-\omega}$. We take a section $e_{1}^{\prime}$ of $E$ such that $e_{1}^{\prime}$ and $e_{2}^{\prime}$ give a frame of $E$. We have the unique decomposition $e_{1}^{\prime}=v_{+}^{\prime}+v_{-}^{\prime}$, where $v_{ \pm}^{\prime}$ are sections of $L_{ \pm \omega}$. By the construction, $L_{ \pm \omega}$ is generated by the images of $e_{1}^{\prime}$ and $e_{2}^{\prime}$. Because $\ell_{P}>0$, we can observe that the image of $v_{ \pm}^{\prime}$ generates $L_{ \pm \omega}$, i.e., $v_{ \pm}^{\prime}$ are frames of $L_{ \pm \omega}$. We may assume that $e_{2}^{\prime}=z^{\ell_{P}} v_{-}^{\prime}$. 
Let $g$ be the holomorphic function determined by $e_{1}^{\prime} \wedge e_{2}^{\prime}=g \cdot s$. Because $g(P) \neq 0$, we can take a holomorphic function $g_{1}$ such that $g_{1}^{2}=g$. We set $e_{i}:=g_{1}^{-1} \cdot e_{i}^{\prime}$ and $v_{ \pm}:=g_{1}^{-1} v_{ \pm}^{\prime}$. Then, they satisfy the desired condition. I

Let $m_{P}$ denote the order of zero of $\omega$ at $P$, i.e., we have $\omega=z^{m_{P}} g_{P}(z) d z$ on $U_{P}$ for a holomorphic function $g_{P}(z)$ with $g_{P}(0) \neq 0$. For the frame $\left(e_{1}, e_{2}\right)$, the Higgs field $\theta_{\mid U_{P}}$ is described as

$$
\theta\left(e_{1}, e_{2}\right)=\left(e_{1}, e_{2}\right)\left(\begin{array}{cc}
\omega & 0 \\
-2 z^{-\ell_{P} \omega} & -\omega
\end{array}\right) \text {. }
$$

In particular, we have $\ell_{P} \leq m_{P}$.

\subsection{The limiting configurations of stable Higgs bundles}

Let $X$ be a compact connected Riemann surface. Let $\left(E, \bar{\partial}_{E}, \theta\right)$ be a Higgs bundle of rank 2 on $X$ such that (i) $\left(E, \bar{\partial}_{E}, \theta\right)$ is stable, (ii) $\left(E, \bar{\partial}_{E}, \theta\right)$ is generically regular semisimple, (iii) $\operatorname{tr}(\theta)=0$. We assume that the spectral curve of $\theta$ is reducible. We obtain a holomorphic 1 -form $\omega \neq 0$ and the line bundles $L_{ \pm \omega}$ as in $\S 4.1$. We impose that $\operatorname{deg}\left(L_{\omega}\right) \leq \operatorname{deg}\left(L_{-\omega}\right)$. We set $L_{1}:=L_{\omega}$ and $L_{2}:=L_{-\omega}$.

We set $d_{i}:=\operatorname{deg}\left(L_{i}\right)(i=1,2)$. We have

$$
d_{1}+d_{2}-\sum_{P \in Z(\omega)} \ell_{P}=\operatorname{deg}(E)
$$

The stability condition for $(E, \theta)$ is equivalent to the inequalities $\operatorname{deg}\left(L_{j} \cap E\right)=d_{j}-\sum_{P \in Z(\omega)} \ell_{P}<\operatorname{deg}(E) / 2$ $(j=1,2)$, i.e.,

$$
d_{j}-\operatorname{deg}(E) / 2>0 \quad(j=1,2) .
$$

The local scaling factor $a_{P}$ of $(E, \bar{\partial}, \theta)$ at $P \in Z(\omega)$ is defined as follows:

$$
a_{P}:=\frac{\ell_{P}}{2\left(m_{P}+1\right)}
$$

We have the functions $\chi_{P}: \mathbb{R}_{\geq 0} \longrightarrow \mathbb{R}_{\leq 0}(P \in Z(\omega))$ given as follows:

$$
\chi_{P}(a):= \begin{cases}\left(m_{P}+1\right)\left(a-a_{P}\right) & \left(0 \leq a \leq a_{P}\right) \\ 0 & \left(a \geq a_{P}\right)\end{cases}
$$

We set $\chi_{E, \theta}(a):=\sum_{P \in Z(\omega)} \chi_{i}(a)$.

Lemma 4.3 We have the unique number $a_{E, \theta}$ satisfying the conditions

$$
d_{1}-\frac{\operatorname{deg}(E)}{2}+\chi_{E, \theta}\left(a_{E, \theta}\right)=0, \quad 0 \leq a_{E, \theta}<\max \left\{a_{P} \mid P \in Z(\omega)\right\} .
$$

Proof The function $\chi_{E, \theta}$ is strictly increasing for $0 \leq a \leq a_{1}:=\max \left\{a_{P} \mid P \in Z(\omega)\right\}$, and we have $\chi_{E, \theta}(a)=0$ for $a \geq a_{1}$. Hence, we have $d_{1}-\frac{\operatorname{deg}(E)}{2}+\chi_{E, \theta}\left(a_{1}\right)=d_{1}-\frac{\operatorname{deg}(E)}{2}>0$. We have $\chi_{E, \theta}(0)=-\sum_{P \in Z(\omega)} \ell_{P} / 2$. Because of $d_{1}+d_{2}-\operatorname{deg}(E)-\sum_{P \in Z(\omega)} \ell_{P}=0$ and $d_{1} \leq d_{2}$, we have $d_{1}-\frac{\operatorname{deg}(E)}{2}+\chi_{E, \theta}(0) \leq 0$. Hence, the claim of the lemma follows.

As in $\S 3.1 .5$, the numbers $-\chi_{P}\left(a_{E, \theta}\right)(P \in Z(\omega))$ give a parabolic structure on the line bundle $L_{1}$. For any $\boldsymbol{c}=\left(c_{P} \mid P \in Z(\omega)\right) \in \mathbb{R}^{Z(\omega)}$, let $\boldsymbol{n}(\boldsymbol{c})=\left(n_{P}(\boldsymbol{c}) \mid P \in Z(\omega)\right) \in \mathbb{Z}^{Z(\omega)}$ be determined by the condition

$$
c_{P}-1<n_{P}(\boldsymbol{c})-\chi_{P}\left(a_{E, \theta}\right) \leq c_{P}
$$

Then, we set $\mathcal{P}_{\boldsymbol{c}}^{\lim }\left(L_{1}\right)=L_{1}\left(\sum_{P \in Z(\omega)} n_{P}(\boldsymbol{c}) P\right)$. We obtain a filtered bundle $\mathcal{P}_{*}^{\lim }\left(L_{1}\right)=\left(\mathcal{P}_{\boldsymbol{c}}^{\lim }\left(L_{1}\right) \mid \boldsymbol{c} \in \mathbb{R}^{Z(\omega)}\right)$ over the meromorphic line bundle $L_{1}(* Z(\omega))$. The parabolic degree of $\mathcal{P}_{*}^{\lim }\left(L_{1}\right)$ is

$$
d_{1}-\sum_{P \in Z(\omega)}\left(-\chi_{P}\left(a_{E, \theta}\right)\right)=d_{1}+\chi_{E, \theta}\left(a_{E, \theta}\right)=\frac{\operatorname{deg}(E)}{2} .
$$


Similarly, the numbers $\chi_{P}\left(a_{E, \theta}\right)+\ell_{P}(P \in Z(\omega))$ determine a filtered bundle over $L_{2}(* Z(\omega))$. The parabolic degree of $\mathcal{P}_{*}^{\lim }\left(L_{2}\right)$ is

$$
d_{2}-\sum_{P \in Z(\omega)}\left(\chi_{P}\left(a_{E, \theta}\right)+\ell_{P}\right)=d_{1}-\frac{\operatorname{deg}(E)}{2}+d_{2}-\sum_{P \in Z(\omega)} \ell_{P}=\frac{\operatorname{deg}(E)}{2} .
$$

The direct $\operatorname{sum} \mathcal{P}_{*}^{\lim } L_{1} \oplus \mathcal{P}_{*}^{\lim } L_{2}$ is called the limiting configuration of $\left(E, \bar{\partial}_{E}, \theta\right)$.

\subsubsection{Hermitian metrics of the limiting configuration}

We fix a Hermitian metric $h_{\operatorname{det}(E)}$ on the determinant line bundle $\operatorname{det}(E)$. We have the 2 -form $R\left(h_{\operatorname{det}(E)}\right)$ obtained as the curvature of the Chern connection of $\left(\operatorname{det}(E), h_{\operatorname{det}(E)}\right)$.

Lemma 4.4 We have Hermitian metrics $h_{L_{j}}^{\lim }$ of the holomorphic line bundles $L_{j \mid X \backslash Z(\omega)}$ satisfying the following conditions:

- The curvature of the Chern connection $\nabla_{j}^{\lim }$ of $\left(L_{j \mid X \backslash Z(\omega)}, h_{L_{j}}^{\lim }\right)$ is equal to $R\left(h_{\operatorname{det}(E)}\right) / 2$.

- For each $P \in Z(\omega)$, let $\left(U_{P}, z_{P}\right)$ be any holomorphic coordinate neighbourhood of $P$ with $z_{P}(P)=0$. Then, $\left|z_{P}\right|^{-2 \chi_{P}\left(a_{E, \theta}\right)} h_{L_{1} \mid U_{P} \backslash\{P\}}$ and $\left|z_{P}\right|^{2 \chi_{P}\left(a_{E, \theta}\right)+2 \ell_{P}} h_{L_{2} \mid U_{P} \backslash\{P\}}$ induce Hermitian metrics of $C^{\infty}$-class on $L_{1 \mid U_{P}}$ and $L_{2 \mid U_{P}}$, respectively.

- Under the isomorphism $\operatorname{det}(E)_{\mid X \backslash Z(\omega)} \simeq\left(L_{1} \otimes L_{2}\right)_{\mid X \backslash Z(\omega)}$, we have $h_{L_{1}}^{\lim } \otimes h_{L_{2}} \lim _{\operatorname{det}(E)}$ on $X \backslash Z(\omega)$.

Proof Because this is standard, we give only a sketch of the proof. We can take Hermitian metrics $h_{L_{j}}(j=1,2)$ of $L_{j \mid X \backslash Z(\omega)}$ such that $\left|z_{P}\right|^{-2 \chi_{P}\left(a_{E, \theta}\right)} h_{L_{1} \mid U_{P} \backslash\{P\}}$ and $\left|z_{P}\right|^{2 \chi_{P}\left(a_{E, \theta}\right)+2 \ell_{P}} h_{L_{2} \mid U_{P} \backslash\{P\}}$ induce Hermitian metrics of $C^{\infty}$-class on $L_{1 \mid U_{P}}$ and $L_{2 \mid U_{P}}$, respectively. Let $R\left(h_{L_{j}}\right)$ denote the curvature form of $\left(L_{j \mid X \backslash Z(\omega)}, h_{L_{j}}\right)$. They naturally induce 2-forms of $C^{\infty}$-class on $X$, which are also denoted by $R\left(h_{L_{j}}\right)$. Because $\frac{\sqrt{-1}}{2 \pi} \int R\left(h_{L_{j}}\right)$ is equal to the parabolic degree of $\mathcal{P}_{*}^{\lim } L_{j}$, we have $\int\left(R\left(h_{L_{j}}\right)-R\left(h_{\operatorname{det}(E)}\right) / 2\right)=0$. We have $C^{\infty}$-functions $\rho_{j}(j=1,2)$ on $X$ such that $\bar{\partial} \partial \rho_{j}=R\left(h_{L_{j}}\right)-R\left(h_{\operatorname{det}(E)}\right) / 2$. We set $h_{L_{j}}^{(1)}:=e^{-\rho_{j}} h_{L_{j}}$. Then, we have $R\left(h_{L_{j}}^{(1)}\right)=R\left(h_{\operatorname{det}(E)}\right) / 2$. Because $\operatorname{det}(E)=L_{1} \otimes L_{2} \otimes \mathcal{O}_{X}\left(-\sum \ell_{P} P\right)$, the tensor product $h_{L_{1}}^{(1)} \otimes h_{L_{2}}^{(2)}$ induces a $C^{\infty}$-Hermitian metric of $\operatorname{det}(E)$. By comparison of the curvature, we have $h_{L_{1}}^{(1)} \otimes h_{L_{2}}^{(2)}=\alpha \cdot h_{\operatorname{det}(E)}$ for a positive constant $\alpha$. Hence, we obtain Hermitian metrics $h_{L_{j}}^{\lim }$ with the desired property by adjusting $h_{L_{j}}^{(1)}$, for example by setting $h_{L_{1}}^{\lim }=\alpha^{-1} h_{L_{1}}^{(1)}$ and $h_{L_{2}}^{\lim }=h_{L_{2}}^{(1)}$.

Such a metric $h_{E, \theta}^{\lim _{1}}:=h_{L_{1}}^{\lim } \oplus h_{L_{2}}^{\lim }$ is also called the limiting configuration of $\left(E, \bar{\partial}_{E}, \theta\right)$. Note that we have the ambiguity of the actions of automorphisms $\alpha \operatorname{id}_{L_{1}} \oplus \alpha^{-1} \operatorname{id}_{L_{2}}(\alpha>0)$, i.e., the pair of $\alpha \cdot h_{L_{1}}^{\lim }$ and $\alpha^{-1} \cdot h_{L_{2}}^{\lim }$ satisfies the conditions in Lemma 4.4. But, the induced Chern connection $\nabla_{E, \theta}^{\lim }:=\nabla_{1}^{\lim } \oplus \nabla_{2}^{\lim }$ on $E_{\mid X \backslash Z(\omega)}$ is well defined. Note that $\nabla_{E, \theta}$ is projectively flat, whose curvature is given by the multiplication of $R\left(h_{\operatorname{det}(E)}\right) / 2$.

Remark 4.5 The metric $h_{E, \theta}$ is also characterized as a Hermitian-Einstein metric for the Higgs bundle $\left(\left(L_{1}, t \omega\right) \oplus\left(L_{2},-t \omega\right)\right)_{\mid X \backslash Z(\omega)}$ adapted to the filtered bundle $\mathcal{P}_{*}^{\lim } L_{1} \oplus \mathcal{P}_{*}^{\lim } L_{2}$ for any $t \neq 0$ such that $\operatorname{det}\left(h_{E, \theta}^{\lim }\right)=$ $h_{\operatorname{det}(E)}$. Because $\left(\mathcal{P}_{*}^{\lim } L_{1}, t \omega\right) \oplus\left(\mathcal{P}_{*}^{\lim } L_{2},-t \omega\right)$ is polystable, we have the ambiguity of the metric $h_{E, \theta}^{\lim }$ by the automorphisms $\alpha \operatorname{id}_{L_{1}} \oplus \alpha^{-1} \mathrm{id}_{L_{2}}$, as usual.

\subsection{The limiting configuration in complementary cases}

Let $X$ be a compact connected Riemann surface. Let $\left(E, \bar{\partial}_{E}, \theta\right)$ be any Higgs bundle of rank 2 on $X$ such that (i) $\left(E, \bar{\partial}_{E}, \theta\right)$ is generically regular semisimple, (ii) $\operatorname{tr}(\theta)=0$. We give limiting configurations in some complementary cases. 


\subsubsection{Polystable Higgs bundles}

Suppose that $\left(E, \bar{\partial}_{E}, \theta\right)$ is polystable. Then, we have the decomposition $(E, \theta)=\left(L_{\omega}, \omega\right) \oplus\left(L_{-\omega},-\omega\right)$. In particular, the spectral curve is reducible, and we have $\ell_{P}=0$ in the description in $\S 4.1$. In this case, we set $(E, \theta)$ as the limiting configuration.

We take a Hermitian metric $h_{\operatorname{det}(E)}$ on the line bundle $\operatorname{det}(E)$. We have Hermitian metrics $h_{L_{ \pm \omega}}^{\lim }$ on $L_{ \pm \omega}$

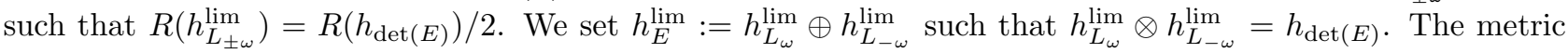
$h_{E, \theta}^{\lim }$ is also called limiting configuration. It is characterized as a Hermitian-Einstein metric on the Higgs bundle $\left(E, \bar{\partial}_{E}, \theta\right)$ such that $\operatorname{det}\left(h_{E, \theta}^{\lim }\right)=h_{\operatorname{det}(E)}$. We have the ambiguity of the metric $h_{E, \theta}^{\lim }$ caused by automorphisms of $(E, \theta)$.

The limiting configuration can also be given as a filtered bundle on $(X, Z(\omega))$ as in the case of stable Higgs bundles $\S 4.2$. We consider the trivial parabolic structures for $L_{ \pm \omega}$ at $P \in Z(\omega)$. Namely, for any $\boldsymbol{c}=\left(c_{P} \mid P \in Z(\omega)\right) \in \mathbb{R}^{Z(\omega)}$, let $\boldsymbol{n}(\boldsymbol{c})=\left(n_{P}(\boldsymbol{c})\right) \in \mathbb{Z}^{Z(\omega)}$ be determined by the condition $c_{P}-1<n_{P}(\boldsymbol{c}) \leq c_{P}$. Then, we set $\mathcal{P}_{\boldsymbol{c}}^{\lim }\left(L_{ \pm \omega}\right)=L_{ \pm \omega}\left(\sum_{P \in Z(\omega)} n_{P}(\boldsymbol{c}) P\right)$. The parabolic degrees of $\mathcal{P}_{*}^{\lim }\left(L_{ \pm \omega}\right)$ is $\operatorname{deg}(E) / 2$. The filtered bundle $\mathcal{P}_{*}^{\lim } L_{\omega} \oplus \mathcal{P}_{*}^{\lim } L_{-\omega}$ is called the limiting configuration of $\left(E, \bar{\partial}_{E}, \theta\right)$.

Remark 4.6 The metric $h_{E, \theta}^{\lim _{\theta}}$ can be characterized as a Hermitian-Einstein metric for $(E, \theta)_{\mid X \backslash Z(\omega)}$ adapted to the filtered bundle such that $\operatorname{det}\left(h_{E, \theta}^{\lim }\right)=h_{\operatorname{det}(E)}$.

\subsubsection{The case where the spectral curve is irreducible}

Suppose that the spectral curve $\Sigma(E, \theta)$ is irreducible. It implies that the Higgs bundle $\left(E, \bar{\partial}_{E}, \theta\right)$ is stable. We take a normalization $\widetilde{X} \longrightarrow \Sigma(E, \theta)$. We have the induced morphism $p: \widetilde{X} \longrightarrow X$, which is a ramified covering of degree 2. We have the involution of $\Sigma(E, \theta)$ induced by the multiplication of -1 on the cotangent bundle $T^{*} X$. It induces an involution $\rho$ of $\widetilde{X}$ over $X$. We can regard $X$ as the quotient space of $\widetilde{X}$ by the action of the group $\{1, \rho\}$.

We set $\left(\widetilde{E}, \bar{\partial}_{\widetilde{E}}, \widetilde{\theta}\right):=p^{*}\left(E, \bar{\partial}_{E}, \theta\right)$. The spectral curve of $(\widetilde{E}, \widetilde{\theta})$ is reducible, i.e., it is the union $\operatorname{Im}(\widetilde{\omega}) \cup \operatorname{Im}(-\widetilde{\omega})$ for a holomorphic one form $\widetilde{\omega}$ on $\widetilde{X}$. We have a natural isomorphism $\rho^{*}\left(\widetilde{E}, \bar{\partial}_{\widetilde{E}}, \widetilde{\theta}\right) \simeq\left(\widetilde{E}, \bar{\partial}_{\widetilde{E}}, \widetilde{\theta}\right)$. By the construction, we have $\rho^{*} \widetilde{\omega}=-\widetilde{\omega}$. We have $Z(\widetilde{\omega})=p^{-1} D(E, \theta)$. We have the line bundles $\widetilde{L}_{\widetilde{\omega}}$ and $\widetilde{L}_{-\widetilde{\omega}}$ on $\widetilde{X}$ with an inclusion $\widetilde{E} \longrightarrow \widetilde{L}_{\widetilde{\omega}} \oplus \widetilde{L}_{-\widetilde{\omega}}$ as in $\S 4$.1. Because $\rho^{*} \widetilde{\omega}=-\widetilde{\omega}$, we have natural isomorphisms $\rho^{*} \widetilde{L}_{ \pm \widetilde{\omega}} \simeq \widetilde{L}_{\mp \widetilde{\omega}}$ such that the following is commutative:

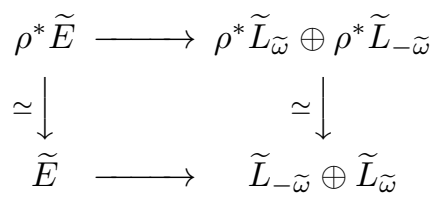

Note that we have already known that $\left(\widetilde{E}, \bar{\partial}_{\widetilde{E}}, \widetilde{\theta}\right)$ is poly-stable. Indeed, because $\left(E, \bar{\partial}_{E}, \theta\right)$ is stable, we have a Hermitian-Einstein metric $h^{H E}$ for $\left(E, \bar{\partial}_{E}, \theta\right)$. The pull back $p^{*} h^{H E}$ is a Hermitian-Einstein metric for $\left(\widetilde{E}, \bar{\partial}_{\widetilde{E}}, \widetilde{\theta}\right)$, which implies the poly-stability of the Higgs bundle.

Stable case When $\left(\widetilde{E}, \bar{\partial}_{\widetilde{E}}, \widetilde{\theta}\right)$ is stable, we obtain the limiting configuration for $\left(\widetilde{E}, \bar{\partial}_{\widetilde{E}}, \widetilde{\theta}\right)$ as a filtered bundle on $(\widetilde{X}, Z(\widetilde{\omega}))$ by the procedure in $\S 4.2$. Namely, we obtain the filtered line bundles $\mathcal{P}_{*}^{\lim } \widetilde{L}_{ \pm \widetilde{\omega}}$ as in $\S 4.2$. In this case, we have $\operatorname{deg}\left(\widetilde{L}_{\widetilde{\omega}}\right)=\operatorname{deg}\left(\widetilde{L}_{-\widetilde{\omega}}\right)$ which implies that $a_{\widetilde{E}, \widetilde{\theta}}=0$ and that the parabolic weights are $-\chi_{P}\left(a_{\widetilde{E}, \widetilde{\theta}}\right)=$ $\chi_{P}\left(a_{\widetilde{E}, \tilde{\theta}}\right)+\ell_{P}=\ell_{P} / 2$. Because $\ell_{P}=\ell_{\rho(P)}$, the isomorphisms $\rho^{*} \widetilde{L}_{ \pm \widetilde{\omega}} \simeq \widetilde{L}_{\mp \widetilde{\omega}}$ induce the isomorphisms of filtered line bundles $\rho^{*} \mathcal{P}_{*}^{\lim } \widetilde{L}_{ \pm \widetilde{\omega}} \simeq \mathcal{P}_{*}^{\lim } \widetilde{L}_{\mp \widetilde{\omega}}$. We have the Hermitian metrics $h_{\widetilde{L}_{ \pm \widetilde{\omega}}}^{\lim }$ of $\widetilde{L}_{ \pm \widetilde{\omega} \mid \widetilde{X} \backslash Z(\widetilde{\omega})}$ as in Lemma 4.4. We may also impose the condition $\rho^{*} h_{\tilde{L}_{\tilde{\omega}}}^{\lim }=h_{\tilde{L}_{-\tilde{\omega}}}^{\lim }$ with which the metrics $h_{\tilde{L}_{ \pm \tilde{\omega}}}^{\lim }$ are uniquely determined.

We set $h_{\widetilde{E}, \widetilde{\theta}}:=h_{\widetilde{L}_{\tilde{\omega}}} \oplus h_{\widetilde{L}_{-} \lim _{-}}$on $\widetilde{E}_{\mid \widetilde{X} \backslash Z(\widetilde{\omega})}$. Because $\rho^{*} h_{\widetilde{E}, \widetilde{\theta}}=h_{\widetilde{E}, \widetilde{\theta}}$, we have the Hermitian metric $h_{E, \theta}$ of $E_{\mid X \backslash D(E, \theta)}$ such that $p^{*} h_{E, \theta} \lim _{\widetilde{E}, \widetilde{\theta}}$. The metric $h_{E, \theta} \lim _{\lim }$ and the associated Chern connection $\nabla_{E, \theta}$ of $E_{\mid X \backslash D(E, \theta)}$ are uniquely determined. 
Remark 4.7 The metric $h_{E, \theta}^{\lim }$ is also characterized as follows. Because the filtered bundle $\mathcal{P}_{*}^{\lim } \widetilde{L}_{\omega} \oplus \mathcal{P}_{*}^{\lim } \widetilde{L}_{-\omega}$ is equivariant with respect to the action of $\{1, \rho\}$, we have the filtered bundle $\mathcal{P}_{*}^{\lim } E$ on $(X, D(E, \theta))$ obtained as the descent of $\mathcal{P}_{*}^{\lim } \widetilde{L}_{\omega} \oplus \mathcal{P}_{*}^{\lim } \widetilde{L}_{-\omega}$. (See $\S 4.3 .3$ below for the descent of filtered bundles in this situation.) We can easily observe that the filtered Higgs bundles $\left(\mathcal{P}_{*}^{\lim } E, t \theta\right)(t \neq 0)$ are stable, and that the parabolic degree is $\operatorname{deg}(E)$. The metric $h_{E, \theta}^{\lim }$ is a unique Hermitian-Einstein metric for the Higgs bundle $\left(E, \bar{\partial}_{E}, t \theta\right)_{\mid X \backslash D(E, \theta)}$ for any $t$, such that $\operatorname{det}\left(h_{E, \theta}^{\lim }\right)=h_{\operatorname{det}(E)}$ adapted to the filtered bundle $\mathcal{P}_{*}^{\lim } E$.

Polystable case We have $\widetilde{E}=\widetilde{L}_{\widetilde{\omega}} \oplus \widetilde{L}_{-\widetilde{\omega}}$. As in $\S 4.3 .1$, we take Hermitian metrics $h_{\widetilde{L}_{ \pm \widetilde{\omega}}}^{\lim }$ of $\widetilde{L}_{ \pm \widetilde{\omega}}$ satisfying $R\left(h_{\widetilde{L}_{ \pm \widetilde{\omega}}}^{\lim }\right)=p^{*} R\left(h_{\operatorname{det}(E)}\right) / 2$ and $h_{\widetilde{L}_{\tilde{\omega}}}^{\lim } \otimes h_{\widetilde{L}_{-\widetilde{\omega}}}^{\lim }=p^{*} h_{\operatorname{det}(E)}$, and we set $h_{\widetilde{E}, \widetilde{\theta}}^{\lim }:=h_{\widetilde{L}_{\widetilde{\omega}}}^{\lim } \oplus h_{\widetilde{L}_{-\widetilde{\omega}}}^{\lim _{\tau^{*}}}$. We also impose the condition $\rho^{*} h_{\widetilde{L}_{\widetilde{\omega}}}^{\lim }=h_{\widetilde{L}_{-\widetilde{\omega}}}^{\lim }$, with which the metrics $h_{\widetilde{L}_{ \pm \widetilde{\omega}}}^{\lim }$ and $h_{\widetilde{E}, \widetilde{\theta}}^{\lim }$ are uniquely determined. Because $\rho^{*} h_{\widetilde{E}, \widetilde{\theta}}^{\lim }$, we have a unique Hermitian metric $h_{E, \theta}^{\lim _{n}}$ of $E$ such that $p^{*} h_{E, \theta}^{\lim _{\theta}}=h_{\widetilde{E}, \widetilde{\theta}}^{\lim }$.

The metric $h_{E, \theta}^{\lim }$ is also characterized as follows.

Lemma 4.8 For any $t \neq 0, h_{E, \theta}^{\lim _{n}}$ is a unique Hermitian-Einstein metric for the stable Higgs bundle $\left(E, \bar{\partial}_{E}, t \theta\right)$ such that $\operatorname{det}\left(h_{E, \theta}^{\lim _{1}}\right)=h_{\operatorname{det}(E)}$. In particular, the Hermitian-Einstein metrics for the Higgs bundles $\left(E, \bar{\partial}_{E}, t \theta\right)$ are independent of $t$.

Proof The claim is clear by the construction of $h_{E, \theta}^{\lim }$.

Remark 4.9 We have the filtered bundle $\mathcal{P}_{*}^{\lim } \widetilde{E}$ as in $\S 4.3 .1$. Because it is equivariant with respect to $\rho$, we obtain a filtered bundle $\mathcal{P}_{*}^{\lim } E$ obtained as the descent of $\mathcal{P}_{*}^{\lim } \widetilde{E}$. The metric $h_{E, \theta}^{\lim }$ is also characterized as a unique Hermitian metric for the stable Higgs bundle $\left(\mathcal{P}_{*}^{\lim } E, t \theta\right)(t \neq 0)$.

\subsubsection{Descent (Appendix)}

Let $\widetilde{X}, X, p, \rho, D(E, \theta)$ and $Z(\widetilde{\omega})$ be as in $\S 4.3 .2$. Let $\widetilde{V}$ be a locally free $\mathcal{O}_{\widetilde{X}}(* Z(\widetilde{\omega}))$-module of finite rank which is equivariant with respect to $\{1, \rho\}$, i.e., we are given an isomorphism $\Phi: \rho^{*} \widetilde{V} \simeq \widetilde{V}$ such that $\Phi \circ \rho^{*} \Phi=$ id. Let $\mathcal{P}_{*} \widetilde{V}$ be a filtered bundle over $\widetilde{V}$, which is equivariant with respect to $\{1, \rho\}$, i.e., $\rho^{*} \mathcal{P}_{\boldsymbol{a}} \widetilde{V}=\mathcal{P}_{\boldsymbol{a}} \widetilde{V}$ for any $\boldsymbol{a} \in \mathbb{R}^{Z(\widetilde{\omega})}$ under the above isomorphism. In this case, the decent of $\mathcal{P}_{*} \widetilde{V}$ is given as follows. By the equivariance of $\widetilde{V}$, we have a locally free $\mathcal{O}_{X}\left(* D(E, \theta)\right.$ )-module $V$ with an isomorphism $p^{*} V \simeq \widetilde{V}$. It is also described as follows. We have the locally free $\mathcal{O}_{X}(* D(E, \theta))$-module $p_{*} \widetilde{V}$. It is equivariant with respect to $\{1, \rho\}$, where the action of $\{1, \rho\}$ on $X$ is trivial. Then, $V$ is the invariant part of $p_{*} \widetilde{V}$ with respect to the action. For any $P \in D(E, \theta)$, let $q(P)$ denote the number of the set $p^{-1}(P)$, which are 1 or 2 . Let $\boldsymbol{a}=\left(a_{P} \mid P \in D(E, \theta)\right) \in \mathbb{R}^{D(E, \theta)}$. For $Q \in p^{-1}(P)$, we set $\widetilde{a}_{Q}:=q(P) \cdot a_{P}$. We obtain the locally free $\mathcal{O}_{\widetilde{X}}$-module $\mathcal{P}_{\widetilde{\boldsymbol{a}}}(\widetilde{V})$. It is equivariant with respect to $\{1, \rho\}$. We obtain the locally free $\mathcal{O}_{X}$-module $p_{*} \mathcal{P}_{\widetilde{\boldsymbol{a}}} \widetilde{V}$. It is equivariant with respect to $\{1, \rho\}$. The invariant part is denoted by $\mathcal{P}_{\boldsymbol{a}} V$. Thus, we obtain a filtered bundle $\mathcal{P}_{*} V$ over $V$, which is the decent of $\mathcal{P}_{*} \widetilde{V}$.

\section{Convergence to the limiting configurations}

\subsection{Statements}

\subsubsection{General case}

Let $\left(E, \bar{\partial}_{E}, \theta\right)$ be a stable Higgs bundle of rank 2 on a compact connected Riemann surface $X$, such that (i) $\left(E, \bar{\partial}_{E}, \theta\right)$ is generically regular semisimple, (ii) the spectral curve is reducible, (iii) $\operatorname{tr}(\theta)=0$. We fix a Hermitian metric $h_{\operatorname{det}(E)}$ of $\operatorname{det}(E)$. We use the notation in $\S 4.2$. We have the limiting configuration $\mathcal{P}_{*}^{\lim } L_{1} \oplus \mathcal{P}_{*}^{\lim } L_{2}$. We take Hermitian metrics $h_{L_{j}}^{\lim }$ for the parabolic line bundle $\mathcal{P}_{*}^{\lim } L_{j}$ satisfying the condition in Lemma 4.4. We set $h_{E, \theta}^{\lim _{1}}:=h_{L_{1}}^{\lim _{1}} \oplus h_{L_{2}}^{\lim _{2}}$. We have the associated Chern connection $\nabla_{E, \theta}^{\lim _{1}}$ of $E_{\mid X \backslash Z(\omega)}$, which is projectively flat.

For any $t>0$, the Higgs bundle $\left(E, \bar{\partial}_{E}, t \theta\right)$ is also stable. We have the Hermitian-Einstein metrics $h_{t}$ of the Higgs bundles $\left(E, \bar{\partial}_{E}, t \theta\right)$, i.e., $R\left(h_{t}\right)+\left[t \theta, t \theta_{h_{t}}^{\dagger}\right]$ is equal to the multiplication of $R\left(h_{\operatorname{det}(E)}\right) / 2$ according 
to Hitchin [6] and Simpson [19]. We impose that $\operatorname{det}\left(h_{t}\right)=h_{\operatorname{det}(E)}$. We have the Chern connection $\nabla_{h_{t}}$ of $\left(E, \bar{\partial}_{E}, h_{t}\right)$.

For any $\gamma>0$, let $\Psi_{\gamma}$ denote the automorphism of $L_{1} \oplus L_{2}$ given by $\Psi_{\gamma}=\gamma \mathrm{id}_{L_{1}} \oplus \gamma^{-1} \mathrm{id}_{L_{2}}$. We define the metric $\Psi_{\gamma}^{*} h_{t}$ of $E_{\mid X \backslash Z(\omega)}$ by $\Psi_{\gamma}^{*} h_{t}\left(u_{1}, u_{2}\right)=h_{t}\left(\Psi_{\gamma} u_{1}, \Psi_{\gamma} u_{2}\right)$ for local sections $u_{i}$ of $E_{\mid X \backslash Z(\omega)}$.

Take any $Q \in X \backslash Z(\omega)$. Let $v_{Q}$ be any frame of $L_{1 \mid Q}$. We set

$$
\gamma(t, Q):=\left(\frac{h_{L_{1}}^{\lim }\left(v_{Q}, v_{Q}\right)}{h_{t}\left(v_{Q}, v_{Q}\right)}\right)^{1 / 2}
$$

We shall prove the following theorem in $§ 5.2-\S 5.4$.

Theorem 5.1 When $t$ goes to $\infty$, the sequence $\Psi_{\gamma(t, Q)}^{*} h_{t}$ converges to $h_{E, \theta}^{\lim }$ in the $C^{\infty}$-sense on any compact subset in $X \backslash Z(\omega)$.

In particular, we obtain the following convergence of unitary connections $\nabla_{h_{t}}$.

Corollary 5.2 The sequence $\nabla_{h_{t}}(t \rightarrow \infty)$ converges to $\nabla_{E, \theta}$ on any compact subset in $X \backslash Z(\omega)$.

Proof If $\operatorname{deg}(E)=0$ and $R\left(h_{\operatorname{det}(E)}\right)=0$, according to the estimates in $\S 2.2$, it is enough to prove that the sequence of the Chern connections of $\left(L_{j \mid X \backslash Z(\omega)}, h_{t \mid L_{j}}\right)$ converges to the Chern connection of $\left(L_{j \mid X \backslash Z(\omega)}, h_{L_{j}}^{\lim }\right)$. It follows from Theorem 5.1.

Let us consider the case where $\operatorname{deg}(E)$ is even. We have a holomorphic line bundle $L_{0}$ on $X$ with an isomorphism $\operatorname{det}(E) \simeq L_{0} \otimes L_{0}$. We have the Hermitian metric $h_{L_{0}}$ of $L_{0}$ such that $h_{\operatorname{det}(E)}=h_{L_{0}} \otimes h_{L_{0}}$ under the isomorphism. Then, $h_{t} \otimes h_{L_{0}}^{-1}$ on $E \otimes L_{0}^{-1}$ is a harmonic metric of $\left(E \otimes L_{0}^{-1}, \bar{\partial}_{E \otimes L_{0}^{-1}}, \theta, h_{t} \otimes h_{L_{0}}^{-1}\right)$. We have the convergence of $\nabla_{h_{t} \otimes h_{L_{0}}^{-1}}$ to the Chern connection of $\left(\left(L_{1} \otimes L_{0}^{-1}\right)_{\mid X \backslash Z(\omega)}, h_{L_{1}}^{\lim } \otimes h_{L_{0}}^{-1}\right) \oplus\left(L_{2} \otimes L_{0}^{-1}\right)_{\mid X \backslash Z(\omega)}, h_{L_{2}}^{\lim } \otimes$ $\left.h_{L_{0}}^{-1}\right)$ ) by the consideration in the case $\operatorname{deg}(E)=0$ and $h_{\operatorname{det}(E)}=0$. Hence, we obtain the convergence of $\nabla_{h_{t}}$ to $\nabla_{E, \theta}$ in the case where $\operatorname{deg}(E)$ is even.

Let us consider the case where $\operatorname{deg}(E)$ is odd. We take a covering map $p: \widetilde{X} \longrightarrow X$ of degree 2 such that $\widetilde{X}$ is connected. Note that $p^{*}\left(E, \bar{\partial}_{E}, \theta\right)$ is stable because $\operatorname{deg}\left(p^{*} L_{i}\right)-\operatorname{deg}\left(p^{*} E\right) / 2>0 . \operatorname{Because} \operatorname{deg}\left(p^{*} E\right)$ is even, we have the convergence of $p^{*} \nabla_{h_{t}}$ to $p^{*} \nabla_{E, \theta}^{\lim }$. Hence, we obtain the convergence of $\nabla_{h_{t}}$ to $\nabla_{E, \theta}^{\lim }$.

\subsubsection{Symmetric case}

We can deduce a stronger result if $X$ and $\left(E, \bar{\partial}_{E}, \theta\right)$ are equipped with an extra symmetry. Suppose that $X$ is equipped with a holomorphic non-trivial involution $\rho$, i.e., $\rho$ is an automorphism of $X$ such that $\rho \circ \rho=\operatorname{id}_{X}$ and $\rho \neq \operatorname{id}_{X}$. Let $\left(E, \bar{\partial}_{E}, \theta\right)$ be as in $§ 5.1 .1$. We impose the following additional conditions.

- $\left(E, \bar{\partial}_{E}, \theta\right)$ is equivariant with respect to the action of $\left\{\operatorname{id}_{X}, \rho\right\}$. Namely, we have an isomorphism $v_{\rho}$ : $\rho^{*}\left(E, \bar{\partial}_{E}, \theta\right) \simeq\left(E, \bar{\partial}_{E}, \theta\right)$ such that $\rho^{*} v_{\rho} \circ v_{\rho}=\mathrm{id}$.

- We have $\rho^{*} \omega=-\omega$.

We impose the condition $\rho^{*} h_{\operatorname{det}(E)}=h_{\operatorname{det}(E)}$ to the metric $h_{\operatorname{det}(E)}$ under the induced isomorphism $\rho^{*} \operatorname{det}(E) \simeq$ $\operatorname{det}(E)$.

The conditions imply that we have natural isomorphisms $\rho^{*} L_{1} \simeq L_{2}$ and $\rho^{*} L_{2} \simeq L_{1}$ which are compatible with $\rho^{*} E \simeq E$. Because $\operatorname{deg}\left(L_{1}\right)=\operatorname{deg}\left(L_{2}\right)$, we have $a_{E, \theta}=0$ and $-\chi_{P}\left(a_{E, \theta}\right)=\chi_{P}\left(a_{E, \theta}\right)+\ell_{P}=\ell_{P} / 2$ for any $P \in Z(\omega)$. We also have $\ell_{P}=\ell_{\rho(P)}$. So, we have natural isomorphisms $\rho^{*} \mathcal{P}_{*}^{\lim } L_{1} \simeq \mathcal{P}_{*}^{\lim } L_{2}$ and $\rho^{*} \mathcal{P}_{*}^{\lim } L_{2} \simeq \mathcal{P}_{*}^{\lim } L_{1}$ compatible with the isomorphism $\rho^{*} E \simeq E$. We can impose the additional condition $\rho^{*} h_{L_{1}}^{\text {lim }}=h_{L_{2}}^{\lim }$ to the conditions in Lemma 4.4 , with which the metrics $h_{L_{i}}^{\lim }$ are uniquely determined. We shall prove the following theorem in $\S 5.5$.

Theorem 5.3 Suppose the symmetric property of $\left(E, \bar{\partial}_{E}, \theta\right)$ as above. For any $t>0$, let $h_{t}$ be the HermitianEinstein metric for $\left(E, \bar{\partial}_{E}, t \theta\right)$ satisfying $\operatorname{det}\left(h_{t}\right)=h_{\operatorname{det}(E)}$. Then, when $t$ goes to $\infty$, the sequence $h_{t}$ is convergent to $h_{E, \theta}^{\lim }$ in the $C^{\infty}$-sense on any compact subset in $X \backslash Z(\omega)$. 


\subsubsection{The case where the spectral curve is irreducible}

As a complement, we explain how to deduce the results in the case where the spectral curve is irreducible, from Theorem 5.3.

Let $\left(E, \bar{\partial}_{E}, \theta\right)$ be a Higgs bundle of rank 2 such that (i) $\left(E, \bar{\partial}_{E}, \theta\right)$ is generically regular semisimple, (ii) the spectral curve $\Sigma(E, \theta)$ is irreducible, (iii) $\operatorname{tr}(\theta)=0$. Note that the Higgs bundle is stable. We fix a Hermitian metric $h_{\operatorname{det}(E)}$ on $\operatorname{det}(E)$. We have the Hermitian-Einstein metrics $h_{t}$ of $\left(E, \bar{\partial}_{E}, t \theta\right)$ such that $\operatorname{det}\left(h_{t}\right)=h_{\operatorname{det}(E)}$. Recall that we have constructed a Hermitian metric $h_{E, \theta}^{\lim }$ of $E_{\mid X \backslash D(E, \theta)}$ in $\S 4.3 .2$.

Corollary 5.4 When $t$ goes to $\infty$, the sequence $h_{t}$ converges to $h_{E, \theta}^{\lim _{\theta}}$ in the $C^{\infty}$-sense on any compact subset in $X \backslash D(E, \theta)$.

Proof We use the notation in $\S 4.3 .2$. If the Higgs bundle $\left(\widetilde{E}, \bar{\partial}_{\widetilde{E}}, \widetilde{\theta}\right)$ is polystable, then the metric $h_{t}$ are independent of $t$ and equal to $h_{E, \theta}^{\lim }$ as remarked in Lemma 4.8. Let us consider the case where $\left(\widetilde{E}, \bar{\partial}_{\widetilde{E}}, \widetilde{\theta}\right)$ is stable. Then, by Theorem 5.3, $p^{*} h_{t}$ is convergent to $h_{\widetilde{E}, \widetilde{\theta}}=p^{*} h_{E, \theta} \lim _{\tilde{X}}$ in the $C^{\infty}$-sense on any compact subset in $\widetilde{X} \backslash Z(\widetilde{\omega})$. Hence, we obtain the convergence of $h_{t}$ to $h_{E, \theta} \lim$.

\subsection{A reduction for the proof of Theorem 5.1}

Let us observe that for the proof of Theorem 5.1 it is enough to consider the case where $\operatorname{deg}(E)=0$ and $R\left(h_{\operatorname{det}(E)}\right)=0$. The argument already appeared in the proof of Corollary 5.2.

Lemma 5.5 Suppose that we have already proved the claim of Theorem 5.1 in the case where $\operatorname{deg}(E)=0$ and $R\left(h_{\operatorname{det}(E)}\right)=0$. Then, we obtain the claim of Theorem 5.1 in the general case.

Proof Let us consider the case where $\operatorname{deg}(E)$ is even. We have a holomorphic line bundle $L_{0}$ with an isomorphism $L_{0}^{\otimes 2} \simeq \operatorname{det}(E)$. We have a Hermitian metric $h_{L_{0}}$ on $L_{0}$ such that $h_{L_{0}} \otimes h_{L_{0}}=h_{\operatorname{det}(E)}$. By the assumption and the construction of the limiting configuration, we obtain that the sequence $\Psi_{\gamma(t, Q)}^{*}\left(h_{t} \otimes h_{L_{0}}^{-1}\right)$ converges to $h_{E, \theta}^{\lim } \otimes h_{L_{0}}^{-1}$ in the $C^{\infty}$-sense on any compact subset in $X \backslash Z(\omega)$. Hence, we obtain the convergence of $\Psi_{\gamma(t, Q)}^{*}\left(h_{t}\right)$ to $h_{E, \theta}^{\lim }$.

Let us consider the case where $\operatorname{deg}(E)$ is odd. We take a covering $p: \widetilde{X} \longrightarrow X$ of degree 2 such that $\widetilde{X}$ is connected. Note that $p^{*}\left(E, \bar{\partial}_{E}, \theta\right)$ is stable because $\operatorname{deg}\left(p^{*} L_{i}\right)-\operatorname{deg}\left(p^{*} E\right) / 2>0$. We take $\widetilde{Q}$ such that $p(\widetilde{Q})=Q$. Because $\operatorname{deg}\left(p^{*} E\right)$ is even, we obtain that the sequence $\Psi_{\gamma(t, \widetilde{Q})}^{*}\left(p^{*} h_{t}\right)$ is convergent to $p^{*} h_{E, \theta} \lim _{\text {in }}$ in the $C^{\infty}$-sense on any compact subset in $\widetilde{X} \backslash p^{-1} D(E, \theta)$. Hence, we obtain the convergence of $\Psi_{\gamma(t, Q)}^{*} h_{t}$ to $h_{E, \theta}$. Thus, the proof of Lemma 5.5 is finished.

It remains to prove Theorem 5.1 in the case where $\operatorname{deg}(E)=0$ and $R\left(h_{\operatorname{det}(E)}\right)=0$, which will be established in $\S 5.3-5.4$.

\subsection{Construction of approximate solutions}

Let $X$ and $\left(E, \bar{\partial}_{E}, \theta\right)$ be the Higgs bundle as in $\S 5.1 .1$. We impose $\operatorname{deg}(E)=0$. We fix a flat metric $h_{\operatorname{det}(E)}$ on $\operatorname{det}(E)$.

\subsubsection{Rescaling around the zeroes}

Let $P \in Z(\omega)$. We take a holomorphic coordinate system $\left(U_{P}, z\right)$ such that the eigenvalues of $\theta$ are $\pm d\left(z^{m_{P}+1}\right)=$ $\pm\left(m_{P}+1\right) z^{m_{P}} d z$. Such $z$ is determined up to the multiplication of a $\left(m_{P}+1\right)$-th square root of 1 . We take frames $v_{i}$ of $L_{i \mid U_{P}}(i=1,2)$ such that (i) $e_{1}=v_{1}+v_{2}$ and $e_{2}=z^{\ell_{P}} v_{2}$ give a frame of $E_{\mid U_{P}}$, (ii) $\left|e_{1} \wedge e_{2}\right|_{h_{\operatorname{det}(E)}}=1$.

We use the Higgs bundle $\left(\widetilde{E}_{\ell}, \widetilde{\theta}\right)$ in $\S 3$ by setting $\alpha=m_{P}+1$. Let $\varphi_{t}: U_{P} \longrightarrow \mathbb{C}$ be given by $\varphi_{t}(z)=$ $t^{1 /\left(m_{P}+1\right)} z=\zeta$. We have

$$
\varphi_{t}^{*} \widetilde{\theta}\left(\varphi_{t}^{*} \widetilde{e}_{1}, \varphi_{t}^{*} \widetilde{e}_{2}\right)=\left(\varphi_{t}^{*} \widetilde{e}_{1}, \varphi_{t}^{*} \widetilde{e}_{2}\right)\left(\begin{array}{cc}
t \alpha z^{m_{P}} d z & 0 \\
-2 \alpha t \cdot t^{-\ell_{P} /\left(m_{P}+1\right)} z^{m_{P}-\ell_{P}} d z & -t \alpha z^{m_{P}} d z
\end{array}\right) .
$$


Hence, we have the isomorphism $\varphi_{t}^{*}\left(\widetilde{E}_{\ell}, \widetilde{\theta}\right) \simeq(E, t \theta)_{\mid U_{P}}$ given by the following correspondence:

$$
t^{\ell_{P} / 2\left(m_{P}+1\right)} \varphi_{t}^{*} \widetilde{e}_{1} \longleftrightarrow e_{1}, \quad t^{-\ell_{P} / 2\left(m_{P}+1\right)} \varphi_{t}^{*} \widetilde{e}_{2} \longleftrightarrow e_{2}
$$

Moreover, we have $t^{\ell_{P} / 2\left(m_{P}+1\right)} \varphi_{t}^{*} \widetilde{v}_{j} \longleftrightarrow v_{j}$

\subsubsection{Local constructions around the zeroes}

In the following, for a given positive function $\nu$, let $O(\nu)$ denote a function $f$ such that $|f| \leq C \nu$, where $C$ is a positive constant independent of $t$. Let $\epsilon$ denote small positive numbers which are independent of $t$.

Let $P \in Z(\omega)$. Suppose that $a_{E, \theta}-\ell_{P} / 2\left(m_{P}+1\right)<0$. We have the harmonic metric $h_{\chi_{P}\left(a_{E, \theta}\right), \ell_{P}}$ of $\left(\widetilde{E}_{\ell}, \widetilde{\theta}\right)$ as in $\S 3.3$. We obtain harmonic metrics $h_{t, P}^{0}:=\varphi_{t}^{*} h_{\chi_{P}\left(a_{E, \theta}\right), \ell_{P}}$ of $\left(E, \bar{\partial}_{E}, t \theta\right)_{\mid U_{P}}$. By construction, we obtain the following from Proposition 3.10:

Lemma 5.6 Take $R_{1, P}>0$ such that $\left\{|z| \leq R_{1, P}\right\} \subset U_{P}$. Take $0<R_{2, P}<R_{1, P}$. On $\left\{R_{2, P} \leq|z|<R_{1, P}\right\} \subset$ $U_{P}$, we have

$$
\begin{gathered}
\left|v_{1}\right|_{h_{t, P}^{0}}=t^{a_{E, \theta}}|z|^{\chi_{P}\left(a_{E, \theta}\right)} \cdot b_{\chi_{P}\left(a_{E, \theta}\right)}\left(1+O\left(\exp \left(-\epsilon|z|^{m_{P}+1} t\right)\right)\right) \\
\left|v_{2}\right|_{h_{t, P}^{0}}=t^{-a_{E, \theta}}|z|^{-\ell_{P}-\chi_{P}\left(a_{E, \theta}\right)} b_{\chi_{P}\left(a_{E, \theta}\right)}^{-1}\left(1+O\left(\exp \left(-\epsilon|z|^{m_{P}+1} t\right)\right)\right) \\
h_{t, P}^{0}\left(v_{1}, v_{2}\right)=O\left(\exp \left(-\epsilon|z|^{m_{P}+1} t\right)\right)
\end{gathered}
$$

We also have the following lemma.

Lemma 5.7 On $\left\{R_{2, P} \leq|z| \leq R_{1, P}\right\}$, we have the following:

$$
\begin{gathered}
\partial \log \left|v_{1}\right|_{h_{t, P}^{0}}^{2}=\chi_{P}\left(a_{E, \theta}\right) d z / z+O(\exp (-\epsilon t)) d z=O(1) d z \\
\partial \log \left|v_{2}\right|_{h_{t, P}^{0}}^{2}=-\left(\ell_{P}+\chi_{P}\left(a_{E, \theta}\right)\right) d z / z+O(\exp (-\epsilon t)) d z=O(1) d z \\
\bar{\partial} \partial \log \left|v_{j}\right|_{h_{t, P}^{0}}^{2}=O(\exp (-\epsilon t)) d z d \bar{z} \quad(j=1,2) \\
\partial h_{t}^{0}\left(v_{1}, v_{2}\right)=O(\exp (-\epsilon t)) d z, \quad \bar{\partial} h_{t}^{0}\left(v_{1}, v_{2}\right)=O(\exp (-\epsilon t)) d \bar{z} \\
\partial \bar{\partial} h_{t}^{0}\left(v_{1}, v_{2}\right)=O(\exp (-\epsilon t)) d z d \bar{z}
\end{gathered}
$$

Proof According to Proposition 3.10, we have $z \partial_{z} \log \left|v_{1}\right|_{h_{t, P}^{0}}^{2}-\chi_{P}\left(a_{E, \theta}\right)=O\left(\exp \left(-\epsilon|z|^{m+1} t\right)\right)$. Hence, we obtain the estimate for $\partial \log \left|v_{1}\right|_{h_{t, P}^{0}}^{2}$ on the domain. We obtain the estimate for $\partial \log \left|v_{2}\right|_{h_{t, P}^{0}}^{2}$ in a similar way. We obtain the estimate for $\bar{\partial} \partial \log \left|v_{j}\right|_{h_{t, P}^{0}}^{2}$ from Lemma 3.11. We obtain the estimate for $\partial h_{t}^{0}\left(v_{1}, v_{2}\right)$ and $\partial \bar{\partial} h_{t}^{0}\left(v_{1}, v_{2}\right)$ from Lemma 3.13 .

Suppose that $a_{E, \theta}-\ell_{P} / 2\left(m_{P}+1\right)=0$. If $\ell_{P}=a_{E, \theta}=0$, we set $h_{t_{i}, P}=\varphi_{t_{i}}^{*} h_{\widetilde{E}_{0}}$, where $h_{\widetilde{E}_{0}}$ is the harmonic metric given in $\S 3.2 .3$. Suppose $\ell_{P}>0$. According to Proposition 3.15 and Proposition 3.19, for a given sequence $t_{i} \rightarrow \infty$, we can take a sequence of negative numbers $c_{i} \rightarrow 0$ such that

$$
\frac{\log b_{c_{i}}}{-c_{i}}=\frac{\log t_{i}}{m_{P}+1}, \quad \text { i.e., } \quad b_{c_{i}} t^{c_{i} /\left(m_{P}+1\right)}=1
$$

We obtain the sequence of harmonic metrics $h_{t_{i}, P}:=\varphi_{t_{i}}^{*} h_{c_{i}, \ell_{P}}$ of $\left(E, \bar{\partial}_{E}, t_{i} \theta\right)_{\mid U_{P}}$, where $h_{c_{i}, \ell_{P}}$ are given as in $\S 3.3$. By Proposition 3.10, we have the following. 
Lemma 5.8 Take $0<R_{2, P}<R_{1, P}$ as in Lemma 5.6. On $\left\{R_{2, P} \leq|z| \leq R_{1, P}\right\}$, we have the following estimates:

$$
\begin{gathered}
\left|v_{1}\right|_{h_{t_{i}, P}^{0}}=t_{i}^{a_{E, \theta}}|z|^{c_{i}}\left(1+O\left(\exp \left(-\epsilon|z|^{m_{P}+1} t_{i}\right)\right)\right) \\
\left|v_{2}\right|_{h_{t_{i}, P}^{0}}=t_{i}^{-a_{E, \theta}}|z|^{-c_{i}-\ell_{P}}\left(1+O\left(\exp \left(-\epsilon|z|^{m_{P}+1} t_{i}\right)\right)\right) \\
h_{t_{i}, P}^{0}\left(v_{1}, v_{2}\right)=O\left(\exp \left(-\epsilon|z|^{m_{P}+1} t_{i}\right)\right)
\end{gathered}
$$

As in the case of Lemma 5.7, we have the following.

Lemma 5.9 Take $0<R_{2, P}<R_{1, P}$. On $\left\{R_{2, P} \leq|z| \leq R_{1, P}\right\}$, we have the following:

$$
\begin{gathered}
\partial \log \left|v_{1}\right|_{h_{t_{i}, P}^{0}}^{2}=c_{i} d z / z+O\left(\exp \left(-\epsilon t_{i}\right)\right) d z=O(1) d z \\
\partial \log \left|v_{2}\right|_{h_{i}, P}^{0}=-\left(c_{i}+\ell_{P}\right) d z / z+O\left(\exp \left(-\epsilon t_{i}\right)\right) d z=O(1) d z \\
\bar{\partial} \partial \log \left|v_{j}\right|_{h_{t_{i}, P}^{0}}^{2}=O\left(\exp \left(-\epsilon t_{i}\right)\right) d z d \bar{z} \\
\partial h_{t_{i}}^{0}\left(v_{1}, v_{2}\right)=O\left(\exp \left(-\epsilon t_{i}\right)\right) d z, \quad \bar{\partial} h_{t_{i}}^{0}\left(v_{1}, v_{2}\right)=O\left(\exp \left(-\epsilon t_{i}\right)\right) d \bar{z} \\
\partial \bar{\partial} h_{t_{i}}^{0}\left(v_{1}, v_{2}\right)=O\left(\exp \left(-\epsilon t_{i}\right)\right) d z d \bar{z}
\end{gathered}
$$

Suppose that $j_{P}:=a_{E, \theta}-\ell_{P} / 2\left(m_{P}+1\right)>0$. We use the notation in $\S 3.4$. For a given sequence $t_{i} \rightarrow \infty$, we set $\kappa_{i}:=t_{i}^{-j_{P} / L}$. We have the Hermitian metrics $h_{\kappa_{i}}$ of $\widetilde{E}_{\ell}$ as in $\S 3.4$. We obtain the Hermitian metrics $h_{t_{i}, P}^{0}:=\varphi_{t_{i}}^{*} h_{\kappa_{i}}$ of $E_{\mid U_{P}}$. By construction, we have the following on $\left\{t_{i}^{-1 /\left(m_{P}+1\right)} \leq|z|<1\right\}$ :

$$
\left|v_{1}\right|_{h_{t_{i}, P}^{0}}=t_{i}^{a_{E, \theta}}, \quad\left|v_{2}\right|_{h_{t_{i}, P}^{0}}=t_{i}^{-a_{E, \theta}}|z|^{-\ell_{P}}, \quad h_{t_{i}, P}^{0}\left(v_{1}, v_{2}\right)=0
$$

Lemma 5.10 Take $0<\epsilon_{P}<<\left(m_{P}+1\right) j_{P}$. We have the following estimate:

$$
\begin{gathered}
R\left(h_{t_{i}, P}^{0}\right)=O\left(t_{i}^{-j_{P}+\epsilon_{P} /\left(m_{P}+1\right)}\right)|z|^{\epsilon_{P}-2} d z d \bar{z} \\
{\left[t_{i} \theta,\left(t_{i} \theta\right)_{h_{t_{i}, P}^{0}}^{\dagger}\right]=O\left(t_{i}^{-j_{P}+\epsilon_{P} /\left(m_{P}+1\right)}\right)|z|^{\epsilon_{P}-2} d z d \bar{z}}
\end{gathered}
$$

Proof Because we have $R\left(h_{t_{i}, P}^{0}\right)=\left[t \theta,(t \theta)_{h_{t_{i}, P}^{0}}^{\dagger}\right]=0$ on $\left\{|z| \geq t^{-1 /\left(m_{P}+1\right)}\right\}$, it is enough to argue the estimates on $|z|<t_{i}^{-1 /\left(m_{P}+1\right)}$. We have the following:

$$
R\left(h_{t_{i}, P}^{0}\right)=O\left(t_{i}^{-j_{P}} t_{i}^{2 /\left(m_{P}+1\right)}\right) d z d \bar{z}, \quad\left[t_{i} \theta,\left(t_{i} \theta\right)_{h_{t_{i}, P}^{0}}^{\dagger}\right]=O\left(t_{i}^{-j_{P}} t_{i}^{2 /\left(m_{P}+1\right)}\right) d z d \bar{z}
$$

Both of them are dominated by

$$
O\left(t_{i}^{-j_{P}+2 /\left(m_{P}+1\right)}\right)|z|^{2-\epsilon_{P}}\left(|z|^{\epsilon_{P}-2} d z d \bar{z}\right)=O\left(t_{i}^{-j_{P}+\epsilon /\left(m_{P}+1\right)}\right)|z|^{\epsilon_{P}-2} d z d \bar{z}
$$

Thus, we obtain the claim of the lemma. 


\subsubsection{Global construction}

We take a Kähler metric $g_{X}$ of $X$. Let $t_{i}$ be any sequence of positive numbers going to $\infty$. We set $\beta_{i}:=t_{i}^{a_{E, \theta}}$. We shall construct a family of Hermitian metrics $h_{t_{i}}^{0}$ of $\left(E, \bar{\partial}_{E}, \theta\right)$ with the following property:

- There exists $p>1$ such that the $L^{p}$-norms of $R\left(h_{t_{i}}^{0}\right)+\left[t_{i} \theta,\left(t_{i} \theta\right)_{h_{t_{i}}^{0}}^{\dagger}\right]$ with respect to $g_{X}$ and $h_{t_{i}}^{0}$ are bounded.

- There exists $C>0$ such that $C^{-1} h_{t_{i}}^{0} \leq h_{t_{i}, P}^{0} \leq C h_{t_{i}}^{0}$ on the neighbourhood $U_{P}$ for each $P \in Z(\omega)$, and that $C^{-1} h_{t_{i}}^{0} \leq \Psi_{\beta_{i}}^{*} h_{E, \theta}^{\lim } \leq C h_{t_{i}}^{0}$ on $X \backslash \bigcup_{P \in Z(\omega)} U_{P}$.

Let $P \in Z(\omega)$. We take $0<R_{2, P}<R_{1, P}$ such that $\left\{|z| \leq R_{1, P}\right\} \subset U_{P}$. We take a function $\rho_{P}: \mathbb{R} \longrightarrow \mathbb{R}_{\geq 0}$ such that $\rho_{P}(s)=1\left(s \leq R_{2, P}\right)$ and $\rho_{P}(s)=0\left(s \geq R_{1, P}\right)$. On $\left\{R_{2, P} \leq|z| \leq R_{1, P}\right\} \subset U_{P}$, we define $h_{t_{i}}^{0}$ by the following conditions:

$$
\begin{gathered}
\log h_{t_{i}}^{0}\left(v_{j}, v_{j}\right)=\rho_{P}(|z|) \log h_{t_{i}, P}^{0}\left(v_{j}, v_{j}\right)+\left(1-\rho_{P}(|z|)\right) \log h_{E, \theta}^{\lim }\left(\Psi_{\beta_{i}} v_{j}, \Psi_{\beta_{i}} v_{j}\right) \quad(j=1,2) \\
h_{t_{i}}^{0}\left(v_{1}, v_{2}\right)=\rho_{P}(|z|) h_{t_{i}, P}^{0}\left(v_{1}, v_{2}\right)
\end{gathered}
$$

Note that $\log h_{t_{i}, P}^{0}\left(v_{j}, v_{j}\right)-\log h_{E, \theta} \lim _{\left(\beta_{i}\right.}\left(v_{j}, \Psi_{\beta_{i}} v_{j}\right)$ are uniformly bounded on $\left\{R_{2, P} \leq|z| \leq R_{1, P}\right\}$. On $\{|z| \leq$ $\left.R_{2, P}\right\}$, we set $h_{t_{i}}^{0}:=h_{t_{i}, P}^{0}$. On $X \backslash \bigcup_{P \in Z(\omega)}\left\{|z| \leq R_{1, P}\right\}$, we set $h_{t_{i}}^{0}:=\Psi_{\beta_{i}}^{*} h_{E, \theta}^{\lim }$. Then, we can check that the family of the Hermitian metrics $h_{t_{i}}^{0}$ has the desired property by using the estimates in $\S 5.3 .2$.

The following lemma is clear by the construction and Proposition 3.14, Proposition 3.20 and Proposition 3.22

Lemma 5.11 The sequence of Hermitian metrics $\Psi_{\beta_{i}^{-1}}^{*} h_{t_{i} \mid X \backslash Z(\omega)}^{0}$ is convergent in the $C^{\infty}$-sense on any compact subset in $X \backslash Z(\omega)$. For the limit $\widetilde{h}_{\infty}^{0}$, the decomposition $L_{1} \oplus L_{2}$ is orthogonal. There exists $M_{1}>0$ such that $M_{1}^{-1} h_{E, \theta} \leq \widetilde{h}_{\infty}^{0} \leq M_{1} h_{E, \theta}^{\lim }$. In particular, there exists $M_{2}>0$ with the following property.

- For any neighbourhood $N$ of $Z(\omega)$, there exists $i_{0}(N)$ such that $M_{2}^{-1} h_{E, \theta} \lim _{\beta_{i}^{-1}} h_{t_{i}}^{0} \leq M_{2} h_{E, \theta}^{\lim }$ on $X \backslash N$ for any $i \geq i_{0}(N)$.

Let $\rho_{i}$ be the self-adjoint endomorphisms of $\left(E_{\mid X \backslash Z(\omega)}, h_{E, \theta}^{\lim }\right)$ determined by $\Psi_{\beta_{i}^{-1}}^{*} h_{t_{i}}^{0}(u, v)=h_{E, \theta}^{\lim _{i}}\left(\rho_{i} u, v\right)$ for any local sections $u$ and $v$. We also have the following.

Lemma 5.12 The sequence $\rho_{i}$ are convergent in the $C^{\infty}$-sense with respect to $h_{E, \theta} \lim _{\text {on }}$ any compact subset in $X \backslash Z(\omega)$. The limit $\rho_{\infty}$ preserves the decomposition $L_{1} \oplus L_{2}$. We have the boundedness of $\rho_{\infty}$ and $\rho_{\infty}^{-1}$ with respect to $h_{E, \theta}$.

\subsection{Proof of Theorem 5.1}

We continue to use the notation in $\S 5.3$.

\subsubsection{Boundedness of a modified sequence}

Let $t_{i} \rightarrow \infty$ be any sequence. It is enough to prove that we can take a subsequence $t_{i}^{\prime}$ such that the sequence $\Psi_{\gamma\left(t_{i}^{\prime}, Q\right)}^{*} h_{t_{i}^{\prime}}$ converges to $h_{E, \theta}^{\lim }$.

Let $\Delta_{X}$ be the Laplacian with respect to the Kähler metric $g_{X}$ of $X$. We construct the family of Hermitian metrics of $h_{t_{i}}^{0}$ on $E$ as in $\S 5.3$. Let $k_{i}$ be the self adjoint endomorphism of $\left(E, h_{t_{i}}^{0}\right)$ determined by $h_{t_{i}}=h_{t_{i}}^{0} k_{i}$, i.e., $h_{t_{i}}(u, v)=h_{t_{i}}^{0}\left(k_{i} u, v\right)$ for local sections $u$ and $v$. According to [19, Proposition 3.1], we have the following on $X$ :

$$
\Delta_{X} \operatorname{Tr}\left(k_{i}\right) \leq\left|\Lambda_{g_{X}} \operatorname{Tr}\left(k_{i} \cdot\left(R\left(h_{t_{i}}^{0}\right)+\left[t_{i} \theta,\left(t_{i} \theta\right)_{h_{t_{i}}^{0}}^{\dagger}\right]\right)\right)\right|
$$


Let $p>1$ be as in $\S 5.3 .3$, i.e., the $L^{p}$-norms of $R\left(h_{t_{i}}^{0}\right)+\left[t_{i} \theta,\left(t_{i} \theta\right)_{h_{t_{i}}^{0}}^{\dagger}\right]$ with respect to $g_{X}$ and $h_{t_{i}}^{0}$ are bounded. We take $q>1$ such that $p^{-1}+q^{-1}<1$. Set $r:=\left(p^{-1}+q^{-1}\right)^{-1}$. Let $\nu_{i}:=\left\|k_{i}\right\|_{L^{q}, h_{t_{i}}^{0}, g_{X}}$ be the $L^{q}$-norm of $k_{i}$ with respect to $h_{t_{i}}^{0}$ and $g_{X}$. Set $s_{i}:=\nu_{i}^{-1} k_{i}$. We have

$$
\Delta_{X} \operatorname{Tr}\left(s_{i}\right) \leq \mid \Lambda_{g_{X}} \operatorname{Tr}\left(s_{i} \cdot\left(R\left(h_{t_{i}}^{0}\right)+\left[t_{i} \theta,\left(t_{i} \theta\right)_{h_{t_{i}}^{0}}^{\dagger}\right]\right) \mid\right.
$$

The $L^{r}$-norms of the right hand side in (37) are bounded for $i$. So we have a constant $C_{1}>0$ and $L_{2}^{r}$-functions $G_{i}$ such that

$$
\Delta_{X}\left(\operatorname{Tr}\left(s_{i}\right)-G_{i}\right) \leq C_{1}, \quad\left\|G_{i}\right\|_{L_{2}^{r}} \leq C_{1} .
$$

Hence, we have $C_{2}>0$ such that $\sup _{X}\left|s_{i}\right|_{h_{t_{i}}^{0}} \leq C_{2}$ holds for any $i$. Again, according to [19, Proposition 3.1], we have the following:

$$
\Delta_{X} \operatorname{Tr}\left(s_{i}\right)=\sqrt{-1} \Lambda_{X} \operatorname{Tr}\left(s_{i} \cdot\left(R\left(h_{t_{i}}^{0}\right)+\left[t_{i} \theta,\left(t_{i} \theta\right)_{h_{t_{i}}^{0}}^{\dagger}\right]\right)\right)-\left|s_{i}^{-1 / 2}\left(\bar{\partial}+t_{i} \theta\right) s_{i}\right|_{h_{t_{i}}^{0}, g_{X}}^{2}
$$

We have a constant $C_{3}>0$ such that $\left|\left(\bar{\partial}_{E}+t_{i} \theta\right) s_{i}\right|_{h_{t_{i}}^{0}, g_{X}}^{2} \leq C_{3}\left|s_{i}^{-1 / 2}\left(\bar{\partial}_{E}+t_{i} \theta\right) s_{i}\right|_{h_{t_{i}}^{0}, g_{X}}^{2}$. Hence, we obtain the following for a constant $C_{4}>0$ :

$$
\int_{X}\left|\bar{\partial}_{E} s_{i}\right|_{h_{t_{i}}^{0}, g_{X}}^{2}+\int_{X}\left|t_{i}\left[\theta, s_{i}\right]\right|_{h_{t_{i}}^{0}, g_{X}}^{2} \leq C_{4}
$$

\subsubsection{Weak convergence of a subsequence}

We consider the sequences of metrics $\bar{h}_{t_{i}}:=\Psi_{\beta_{i}^{-1}}^{*} h_{t_{i}}$ and $\bar{h}_{t_{i}}^{0}:=\Psi_{\beta_{i}^{-1}}^{*} h_{t_{i}}^{0}$ on $E_{\mid X \backslash Z(\omega)}$. Let $\bar{k}_{i}$ be the self-adjoint endomorphism of $\left(E_{\mid X \backslash Z(\omega)}, \bar{h}_{t_{i}}^{0}\right)$ determined by $\bar{h}_{t_{i}}=\bar{h}_{t_{i}}^{0} \bar{k}_{i}$. We have $\left\|\bar{k}_{i}\right\|_{L^{q}, \bar{h}_{t_{i}}^{0}, g_{X}}=\nu_{i}$. Set $\bar{s}_{i}:=\nu_{i}^{-1} \bar{k}_{i}$. We have

$$
\sup _{X}\left|\bar{s}_{i}\right|_{\bar{h}_{t_{i}}^{0}} \leq C_{2}
$$

We also have

$$
\int_{X}\left|\bar{\partial}_{E} \bar{s}_{i}\right|_{\bar{h}_{t_{i}}^{0}, g_{X}}^{2}+\int_{X}\left|t_{i}\left[\theta, \bar{s}_{i}\right]\right|_{\bar{h}_{t_{i}}^{0}, g_{X}}^{2} \leq C_{4}
$$

By Lemma 5.11 and (39), we may assume that the sequence $\bar{s}_{i}$ is weakly convergent in $L_{1}^{2}$ on any compact subset in $X \backslash Z(\omega)$ with respect to $g_{X}$ and $h_{E, \theta}^{\lim }$. Let $\bar{s}_{\infty}$ denote the weak limit.

Lemma $5.13 \bar{s}_{\infty}$ is bounded with respect to $h_{E, \theta}^{\lim _{1}}$. We have $\bar{s}_{\infty} \neq 0$.

Proof By Lemma 5.12 and (38), there exists $M_{3}>0$ with the following property.

- For any neighbourhood $N$ of $Z(\omega)$, there exists $i_{3}(N)$ such that $\left|\bar{s}_{i}\right|_{h_{E, \theta}^{\lim }} \leq M_{3}$ on $X \backslash N$ for any $i \geq i_{3}(N)$.

Hence, we have the boundedness $\left|\bar{s}_{\infty}\right|_{h_{E, \theta}^{\lim }} \leq M_{3}$.

Take a small $\delta>0$. By (38), we have a small neighbourhood $N_{1}$ of $Z(\omega)$ such that

$$
\int_{X \backslash N_{1}}\left|\bar{s}_{i}\right|_{\bar{h}_{t_{i}}^{0}}^{q} \operatorname{dvol}_{g_{X}} \geq 1-\delta>0
$$

Hence, we obtain $\int_{X \backslash N_{1}}\left|\bar{s}_{\infty}\right|_{h_{E, \theta}^{\lim }}^{q} \operatorname{dvol}_{g_{X}}>0$. In particular, we have $\bar{s}_{\infty} \neq 0$.

Lemma 5.14 We have $\left[\bar{s}_{\infty}, \theta\right]=0$. In particular, $\bar{s}_{\infty}$ preserves the decomposition $L_{1} \oplus L_{2}$.

Proof We have $\lim _{i \rightarrow \infty} \int_{X}\left|\left[\bar{s}_{i}, \theta\right]\right|_{\bar{h}_{t_{i}}^{0}, g_{X}}^{2}=0$ from (38), which implies the claim of the lemma. 


\subsubsection{Modification}

Let $\rho_{i}$ be as in Lemma 5.12. We set $\bar{s}_{i}^{1}:=\rho_{i} \circ \bar{s}_{i}$. It is self-adjoint with respect to $h_{E, \theta}^{\lim }$, and we have $\nu_{i}^{-1} \Psi_{\beta_{i}^{-1}}^{*} h_{t_{i}}=h_{E, \theta}^{\lim } \cdot \bar{s}_{i}^{1}$. The sequence $\bar{s}_{i}^{1}$ is weakly convergent in $L_{1}^{2}$ on any compact subset in $X \backslash Z(\omega)$. Let $\bar{s}_{\infty}^{1}$ denote the weak limit. We have $\bar{s}_{\infty}^{1}=\rho_{\infty} \circ \bar{s}_{\infty}$. We obtain the following from Lemma 5.12, Lemma 5.13 and Lemma 5.14 .

Lemma $5.15 \bar{s}_{\infty}^{1}$ is bounded with respect to $h_{E, \theta} \lim _{\text {. We have }} \bar{s}_{\infty}^{1} \neq 0$ and $\left[\bar{s}_{\infty}^{1}, \theta\right]=0$.

Lemma 5.16 We have $\bar{\partial}_{E} \bar{s}_{\infty}^{1}=0$.

Proof Applying [19, Proposition 3.1] to $h_{\infty}^{\lim }$ and $\nu_{i}^{-1} \Psi_{\beta_{i}^{-1}}^{*} h_{t_{i}}$, we obtain the following on $X \backslash Z(\omega)$ :

$$
\left|\left(\bar{s}_{i}^{1}\right)^{-1 / 2} \bar{\partial}_{E} \bar{s}_{i}^{1}\right|_{h_{E, \theta}^{\lim , g_{X}}}^{2} \leq\left|\left(\bar{s}_{i}^{1}\right)^{-1 / 2}\left(\bar{\partial}+t_{i} \theta\right) \bar{s}_{i}^{1}\right|_{h_{E, \theta}^{\lim , g_{X}}}^{2}=-\sqrt{-1} \Lambda_{g_{X}} \bar{\partial} \partial \operatorname{Tr}\left(\bar{s}_{i}^{1}\right)
$$

We take a $C^{\infty}$-function $\mu: \mathbb{R} \longrightarrow \mathbb{R}_{\geq 0}$ such that $\mu(s)=1(s \leq 1)$ and $\mu(s)=0(s \geq 2)$. For any sufficiently large real number $T$, let $\chi_{T}: X \longrightarrow \mathbb{R}_{\geq 0}$ be the $C^{\infty}$-function such that (i) $\chi_{T} \equiv 1$ on $X \backslash \bigcup_{P \in Z(\omega)} U_{P}$, (ii) $\chi_{T}(z)=\mu\left(-T^{-1} \log |z|\right)$ on the coordinate neighbourhoods $\left(U_{P}, z\right)$ for $P \in Z(\omega)$.

We have a constant $M_{4}>0$ with the following property.

- For any neighbourhood $N$ of $Z(\omega)$, there exists $i_{4}(N)$ such that $\left|\bar{s}_{i}^{1}\right|_{h_{E, \theta}^{\lim }} \leq M_{4}$ on $X \backslash N$ for any $i \geq i_{4}(N)$.

Then, we have a constant $C_{10}$ such that for any fixed $T>0$ the following holds for a large $i$ :

$$
\begin{aligned}
\int \chi_{T}\left|\bar{\partial}_{E} \bar{s}_{i}^{1}\right|_{h_{E, \theta}^{\lim }, g_{X}}^{2} \operatorname{dvol}_{g_{X}} \leq C_{10} \int \chi_{T}\left|\left(\bar{s}_{i}^{1}\right)^{-1 / 2} \bar{\partial}_{E} \bar{s}_{i}^{1}\right|_{h_{E, \theta}^{\lim }, g_{X}}^{2} \operatorname{dvol}_{g_{X}} \\
\quad \leq C_{10}\left|\int \chi_{T} \bar{\partial} \cdot \partial \operatorname{Tr}\left(\bar{s}_{i}^{1}\right)\right|=C_{10}\left|\int\left(\bar{\partial} \partial \chi_{T}\right) \cdot \operatorname{Tr}\left(\bar{s}_{i}^{1}\right)\right|
\end{aligned}
$$

By taking the limit for $i \rightarrow \infty$, we obtain

$$
\int \chi_{T}\left|\bar{\partial}_{E} \bar{s}_{\infty}^{1}\right|_{h_{E, \theta}^{1 \lim , g_{X}}}^{2} \operatorname{dvol}_{g_{X}} \leq C_{10}\left|\int\left(\bar{\partial} \partial \chi_{T}\right) \cdot \operatorname{Tr}\left(\bar{s}_{\infty}^{1}\right)\right|
$$

Note that we have already known the boundedness of $\operatorname{Tr}\left(\bar{s}_{\infty}^{1}\right)$. We also have the uniform boundedness of $\bar{\partial} \partial \chi_{T}$ with respect to the Poincaré like metric on $X \backslash Z(\omega)$. Hence, by taking the limit for $T \rightarrow \infty$, we obtain

$$
\int\left|\bar{\partial}_{E} \bar{s}_{\infty}^{1}\right|_{h_{E, \theta}^{\lim }, g_{X}}^{2} \operatorname{dvol}_{g_{X}} \leq 0
$$

Hence, we obtain $\bar{\partial}_{E} \bar{s}_{\infty}^{1}=0$.

By Lemma 5.15 and Lemma 5.16, we have $\bar{s}_{\infty}^{1}=\alpha_{1} \operatorname{id}_{L_{1}} \oplus \alpha_{2} \operatorname{id}_{L_{2}}$ for non-negative real numbers $\alpha_{i}(i=1,2)$. We have $\left(\alpha_{1}, \alpha_{2}\right) \neq(0,0)$.

\subsubsection{End of the proof of Theorem 5.1}

Suppose that $\alpha_{1} \neq 0$. Let $u_{j}$ be local frames of $L_{j}(j=1,2)$ on a relatively compact open subset in $X \backslash Z(\omega)$. We set $\gamma_{i}:=\beta_{i}^{-1} \nu_{i}^{-1 / 2} \alpha_{1}^{-1 / 2}$. Then, we have the following:

$$
\lim _{i \rightarrow \infty}\left|u_{1}\right|_{h_{t_{i}}} \gamma_{i}=\left|u_{1}\right|_{h_{L_{1}}^{\mathrm{lim}}}
$$

By the asymptotic orthogonality in $\S 2$, we have the following on $X \backslash N$, where $N$ is any neighbourhood of $Z(\omega)$ :

$$
\left|u_{1} \wedge u_{2}\right|_{\operatorname{det}(E)}^{2}=\left|u_{1}\right|_{h_{t_{i}}}^{2}\left|u_{2}\right|_{h_{t_{i}}}^{2}-\left|h_{t_{i}}\left(u_{1}, u_{2}\right)\right|^{2}=\left|u_{1}\right|_{h_{t_{i}}}^{2}\left|u_{2}\right|_{h_{t_{i}}}^{2} \cdot\left(1+O\left(\exp \left(-\epsilon t_{i}\right)\right)\right)
$$


We also have $\left|u_{1} \wedge u_{2}\right|_{\operatorname{det}(E)}=\left|u_{1}\right|_{h_{E, \theta}^{\lim }} \cdot\left|u_{2}\right|_{h_{E, \theta}^{\text {lim }}}$. Hence, we obtain the following from (41):

$$
\lim _{i \rightarrow \infty}\left|u_{2}\right|_{h_{t_{i}}} \gamma_{i}^{-1}=\left|u_{2}\right|_{h_{L_{2}}^{\lim }}
$$

We obtain the following for $j=1,2$ from (41) and (42):

$$
\lim _{i \rightarrow \infty} \Psi_{\gamma_{i}}^{*} h_{t_{i}}\left(u_{j}, u_{j}\right)=h_{E, \theta}^{\lim _{i}}\left(u_{j}, u_{j}\right)
$$

We also have the following on $X \backslash N$, where $N$ is any neighbourhood of $Z(\omega)$ :

$$
\Psi_{\gamma_{i}}^{*} h_{t_{i}}\left(u_{1}, u_{2}\right)=h_{t_{i}}\left(u_{1}, u_{2}\right)=O\left(\exp \left(-\epsilon t_{i}\right)\right) \cdot\left|u_{1}\right|_{h_{t_{i}}} \cdot\left|u_{2}\right|_{h_{t_{i}}}=O\left(\exp \left(-\epsilon t_{i}\right)\right) \cdot\left|u_{1}\right|_{\Psi_{\gamma_{i}}^{*} h_{t_{i}}} \cdot\left|u_{2}\right|_{\Psi_{\gamma_{i}}^{*}} h_{t_{i}}
$$

So, we obtain $\lim _{i \rightarrow \infty} \Psi_{\gamma_{i}}^{*} h_{t_{i}}\left(u_{1}, u_{2}\right)=0=h_{E, \theta}^{\lim }\left(u_{1}, u_{2}\right)$. Hence, we have the convergence of $\Psi_{\gamma_{i}}^{*} h_{t_{i}}$ to $h_{E, \theta}^{\lim }$ in $C^{0}$ on any compact subset in $X \backslash Z(\omega)$.

By the construction of the sequence $\gamma\left(t_{i}, Q\right)$, we have $\lim _{i \rightarrow \infty} \gamma\left(t_{i}, Q\right) \cdot \gamma_{i}^{-1}=1$. Hence, we have the convergence of $\Psi_{\gamma\left(t_{i}, Q\right)}^{*} h_{t_{i}}$ to $h_{E, \theta}^{\lim }$ in $C^{0}$ on any compact subset in $X \backslash Z(\omega)$. We can obtain the convergence of the higher derivative from Corollary 2.15. Thus, we are done in the case $\alpha_{1} \neq 0$.

We can argue the case $\alpha_{2} \neq 0$ in a similar way. Thus, the proof of Theorem 5.1 is finished.

\subsection{Proof of Theorem 5.3}

\subsubsection{Preliminary}

Let $\left(E, \bar{\partial}_{E}, \theta\right)$ be any stable Higgs bundle of rank 2 on $X$ such that (i) $\left(E, \bar{\partial}_{E}, \theta\right)$ is generically regular semisimple, (ii) the spectral curve $\Sigma(E, \theta)$ is reducible, (iii) $\operatorname{tr}(\theta)=0$. We have holomorphic line bundles $L_{i}(i=1,2)$ with an inclusion $E \longrightarrow L_{1} \oplus L_{2}$ as in $\S 4.2$. We assume that $\operatorname{deg}\left(L_{1}\right)=\operatorname{deg}\left(L_{2}\right)$. Then, we have $a_{E, \theta}=0$ and $-\chi_{P}\left(a_{E, \theta}\right)=\chi_{P}\left(a_{E, \theta}\right)+\ell_{P}=\ell_{P} / 2$.

We fix a Hermitian metric $h_{\operatorname{det}(E)}$ of $\operatorname{det}(E)$. For any $t>0$, we have Hermitian-Einstein metrics $h_{t}$ for $\left(E, \bar{\partial}_{E}, t \theta\right)$ such that $\operatorname{det}\left(h_{t}\right)=h_{\operatorname{det}(E)}$. We have the metric $h_{E, \theta}^{\lim }$ as in $\S 5.1 .1$.

We take any sequence $t_{i} \rightarrow \infty$. Let $k_{i}^{(2)}$ be the self-adjoint endomorphisms of $\left(E_{\mid X \backslash Z(\omega)}, h_{E, \theta}^{\lim }\right)$ determined by $h_{t_{i}}=h_{E, \theta}^{\lim _{E}} \cdot k_{i}^{(2)}$.

Lemma 5.17 After going to a subsequence $\{i(p)\} \subset\{i\}$ there exists a sequence of positive numbers $\nu_{i(p)}$ such that the sequence $\nu_{i(p)}^{-1} k_{i(p)}^{(2)}$ weakly converges to a morphism $\alpha_{1} \cdot \mathrm{id}_{L_{1}} \oplus \alpha_{2} \cdot \mathrm{id}_{L_{2}}$ in $L_{1}^{2}$ locally on $X \backslash Z(\omega)$ for non-negative real numbers $\alpha_{j}(j=1,2)$ with $\left(\alpha_{1}, \alpha_{2}\right) \neq(0,0)$.

Proof Let us consider the case where $\operatorname{deg}(E)$ is even. We take a holomorphic line bundle $L_{0}$ with an isomorphism $L_{0} \otimes L_{0} \simeq \operatorname{det}(E)$. We have a Hermitian metric $h_{L_{0}}$ such that $h_{L_{0}} \otimes h_{L_{0}}=h_{\operatorname{det}(E)}$. We have the harmonic metrics $h_{E \otimes L_{0}^{-1}, t_{i}}$ for $\left(E \otimes L_{0}^{-1}, \bar{\partial}_{E \otimes L_{0}^{-1}}, t_{i} \theta\right)$. We have $h_{E \otimes L_{0}^{-1}, t_{i}}=h_{t_{i}} \otimes h_{L_{0}}^{-1}$ and $h_{E \otimes L_{0}^{-1}, \theta}^{\lim }=h_{E, \theta}^{\lim } \otimes h_{L_{0}}^{-1}$. Hence, $k_{i}^{(2)}$ is the self-adjoint endomorphisms of $\left(\left(E \otimes L_{0}^{-1}\right)_{\mid X \backslash Z(\omega)}, h_{E \otimes L_{0}^{-1}, \theta}^{\lim }\right)$ determined by $h_{E \otimes L_{0}^{-1}, t_{i}}=h_{E \otimes L_{0}^{-1}, \theta}^{\lim } \cdot k_{i}^{(2)}$. Note that $a_{E \otimes L_{0}^{-1}, \theta}=0$ and $\beta_{i}=t_{i}^{{ }^{a}{ }{ }^{-1} L_{0}^{-1}}=1$ in $\S 5.3 .3$ and $\S 5.4$. Then, the claim of the lemma for $k_{i}^{(2)}$ has been already observed in $\S 5.4 .2-\S 5.4 .3$. We can easily reduce the case where $\operatorname{deg}(E)$ is odd to the case where $\operatorname{deg}(E)$ is even, by taking the pull back by a covering $\widetilde{X} \longrightarrow X$ of degree 2 .

\subsubsection{Proof of Theorem 5.3}

We take any sequence $t_{i} \rightarrow \infty$. It is enough to prove that we can take a subsequence $t_{i}^{\prime}$ such that $h_{t_{i}^{\prime}}$ converges to $h_{E, \theta}^{\lim }$ on any compact subsets in $X \backslash Z(\omega)$.

Let $k_{i}^{(2)}$ be the self-adjoint endomorphisms of $\left(E_{\mid X \backslash Z(\omega)}, h_{E, \theta}^{\lim }\right)$ determined by $h_{t_{i}}=h_{E, \theta}^{\lim _{i}} \cdot k_{i}^{(2)}$, as in $\S 5.5 .1$. Because $\rho^{*} h_{E, \theta}^{\lim _{n}}=h_{E, \theta}^{\lim _{n}}$ and $\rho^{*} h_{t_{i}}=h_{t_{i}}$, we have $\rho^{*} k_{i}^{(2)}=k_{i}^{(2)}$. As remarked in Lemma 5.17 , by going to a subsequence, we may assume to have a sequence of positive numbers $\nu_{i}$ such that the sequence $\nu_{i}^{-1} k_{i}^{(2)}$ is 
weakly convergent to $\alpha_{1} \operatorname{id}_{L_{1}} \oplus \alpha_{2} \operatorname{id}_{L_{2}}$ in $L_{1}^{2}$ locally on $X \backslash Z(\omega)$, where $\alpha_{i}$ are non-negative numbers such that $\left(\alpha_{1}, \alpha_{2}\right) \neq(0,0)$. Because $\rho^{*}\left(\nu_{i}^{-1} k_{i}^{(2)}\right)=\nu_{i}^{-1} k_{i}^{(2)}, \rho^{*}\left(L_{1}\right)=L_{2}$ and $\rho^{*}\left(L_{2}\right)=L_{1}$, we have $\alpha_{1}=\alpha_{2}$. In particular, $\alpha_{1} \cdot \alpha_{2} \neq 0$. Because $\operatorname{det}\left(k_{i}^{(2)}\right)=1$, the sequence $\nu_{i}^{-2}$ converges to $\alpha_{1} \cdot \alpha_{2}$. In particular, the sequences $\nu_{i}$ and $\nu_{i}^{-1}$ are bounded.

We take a subsequence $h_{t_{i(p)}}$ for which the sequence $k_{i(p)}^{(2)}$ is convergent to $\beta \operatorname{id}_{L_{1}} \oplus \beta \mathrm{id}_{L_{2}}$ for a positive number $\beta$. But, we have $\operatorname{det}\left(k_{i(p)}^{(2)}\right)=1$ and hence $\beta=1$, i.e., $k_{i(p)}^{(2)}$ is convergent to the identity id, indeed. Hence, we can conclude that the sequence $k_{i}^{(2)}$ is convergent to id, and the proof of Theorem 5.3 is finished.

\section{References}

[1] L. V. Ahlfors, An extension of Schwarz's lemma, Trans. Amer. Math. Soc. 43 (1938), 359-364.

[2] O. Biquard and P. Boalch, Wild non-abelian Hodge theory on curves, Compos. Math. 140 (2004), 179-204.

[3] B. Collier, Q. Li. Asymptotics of certain families of Higgs bundles in the Hitchin component, arXiv:1405.1106

[4] D. Gaiotto, G. Moore, A. Neitzke, Four-dimensional wall-crossing via three-dimensional field theory, Comm. Math. Phys. 299 (2010), 163-224

[5] D. Gaiotto, G. Moore, A. Neitzke, Wall-crossing, Hitchin systems, and the WKB approximation, Adv. Math. 234 (2013), 239-403.

[6] N. Hitchin, The self-duality equations on a Riemann surface, Proc. London Math. Soc. (3) 55 (1987), $59-126$.

[7] L. Katzarkov, A. Noll, P. Pandit, C. Simpson, Harmonic Maps to Buildings and Singular Perturbation Theory, arXiv:1311.7101

[8] S. Lang, Real Analysis, Second edition. Addison-Wesley Publishing Company, Advanced Book Program, Reading, MA, 1983

[9] M. Maruyama, K. Yokogawa, Moduli of parabolic stable sheaves, Math. Ann. 293 (1992), 77-99.

[10] V. B. Mehta, C. S. Seshadri, Moduli of vector bundles on curves with parabolic structures, Math. Ann. 248 (1980), 205-239

[11] R. Mazzeo, J. Swoboda, H. Weiss, F. Witt. Ends of the moduli space of Higgs bundles, arXiv:1405.5765

[12] R. Mazzeo, J. Swoboda, H. Weiss, F. Witt, Limiting configurations for solutions of Hitchin's equation, arXiv:1502.01692, Semin. Theor. Spectr. Geom. 31 (2012-2014), 91-116.

[13] T. Mochizuki, Asymptotic behaviour of tame harmonic bundles and an application to pure twistor Dmodules I, II, Mem. AMS. 185 (2007)

[14] T. Mochizuki, Kobayashi-Hitchin correspondence for tame harmonic bundles and an application, Astérisque 309 (2006), viii+117 pp.

[15] T. Mochizuki, Kobayashi-Hitchin correspondence for tame harmonic bundles II, Geometry\&Topology 13, (2009), 359-455, math.DG/0602266

[16] T. Mochizuki, Wild harmonic bundles and wild pure twistor D-modules, Astérisque 340, Société Mathématique de France, Paris, 2011.

[17] T. Mochizuki, Harmonic bundles and Toda lattices with opposite sign, arXiv:1301.1718. (The second half of this preprint was published as [18].) 
[18] T. Mochizuki, Harmonic bundles and Toda lattices with opposite sign II, Comm. Math. Phys. 328, (2014), 1156-1198

[19] C. T. Simpson, Constructing variations of Hodge structure using Yang-Mills theory and applications to uniformization, J. Amer. Math. Soc. 1 (1988), 867-918.

[20] C. T. Simpson, Harmonic bundles on noncompact curves, J. Amer. Math. Soc. 3 (1990), 713-770.

[21] C. T. Simpson, Higgs bundles and local systems, Publ. I.H.E.S., 75 (1992), 5-95.

[22] C. T. Simpson, The Hodge filtration on nonabelian cohomology, Proc. Sympos. Pure Math., 62, Part 2, Amer. Math. Soc., Providence, RI, (1997), 217-281.

Address

Research Institute for Mathematical Sciences, Kyoto University, Kyoto 606-8502, Japan, takuro@kurims.kyoto-u.ac.jp 